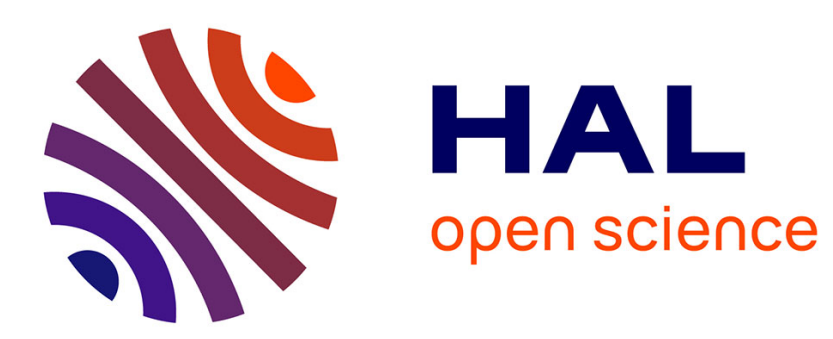

\title{
An asymptotic plate model for magneto-electro-thermo-elastic sensors and actuators
} Francesco Bonaldi, G. Geymonat, F. Krasucki, M. Serpilli

\section{To cite this version:}

Francesco Bonaldi, G. Geymonat, F. Krasucki, M. Serpilli. An asymptotic plate model for magnetoelectro-thermo-elastic sensors and actuators. Mathematics and Mechanics of Solids, 2017, 22 (4), pp.798-822. 10.1177/1081286515612885 . hal-01207392

\section{HAL Id: hal-01207392 \\ https://hal.science/hal-01207392}

Submitted on 1 Oct 2015

HAL is a multi-disciplinary open access archive for the deposit and dissemination of scientific research documents, whether they are published or not. The documents may come from teaching and research institutions in France or abroad, or from public or private research centers.
L'archive ouverte pluridisciplinaire HAL, est destinée au dépôt et à la diffusion de documents scientifiques de niveau recherche, publiés ou non, émanant des établissements d'enseignement et de recherche français ou étrangers, des laboratoires publics ou privés. 


\title{
AN ASYMPTOTIC PLATE MODEL FOR MAGNETO-ELECTRO-THERMO-ELASTIC SENSORS AND ACTUATORS
}

\author{
FRANCESCO BONALDI \\ Institut Montpelliérain Alexander Grothendieck, UMR-CNRS 5149, \\ Université de Montpellier, Place Eugène Bataillon, 34095 Montpellier Cedex 5, France \\ francesco.bonaldi@univ-montp2.fr \\ GIUSEPPE GEYMONAT \\ Laboratoire de Mécanique des Solides, UMR-CNRS 7649, \\ École Polytechnique, Route de Saclay, 91128 Palaiseau Cedex, France \\ giuseppe.geymonat@lms.polytechnique.fr \\ FRANÇOISE KRASUCKI \\ Institut Montpelliérain Alexander Grothendieck, UMR-CNRS 5149, \\ Université de Montpellier, Place Eugène Bataillon, 34095 Montpellier Cedex 5, France \\ francoise.krasucki@univ-montp2.fr \\ MICHELE SERPILLI \\ Department of Civil and Building Engineering, and Architecture, \\ Polytechnic University of Marche, Via Brecce Bianche, 60131 Ancona, Italy \\ m.serpilli@univpm.it
}

\begin{abstract}
We present an asymptotic two-dimensional plate model for linear magnetoelectro-thermo-elastic sensors and actuators, under the hypotheses of anisotropy and homogeneity. Four different boundary conditions pertaining to electromagnetic quantities are considered, leading to four different models: the sensor-actuator model, the actuatorsensor model, the actuator model and the sensor model. We validate the obtained twodimensional models by proving weak convergence results. Each of the four plate problems turns out to be decoupled into a flexural problem, involving the transversal displacement of the plate, and a certain partially or totally coupled membrane problem.
\end{abstract}

Keywords: sensors; actuators; plates; piezoelectricity; magnetostriction; couplings; asymptotic analysis; magnetoelasticity; thermal effects; pyroelectricity; pyromagnetism.

Mathematics Subject Classification 2000: 74F99, 74K20, 74H10

\section{Introduction}

In this paper, we consider a linear model of magneto-electro-thermo-elastic plates, behaving either as piezoelectric sensors or piezomagnetic actuators, based on the quasi-static assumption on the electric and magnetic fields, whereby both fields can be expressed as gradients of the corresponding potentials. This assumption was justified by means of a nondimensionalization of the equations governing the problem in its general setting in [2], 
wherein a proof of well-posedness for this problem, along with its quasi-static counterpart, was also accomplished.

The behavior of the plate-like body under study, as to whether it represents a sensor or an actuator, of piezoelectric or piezomagnetic nature, is determined by four different sets of boundary conditions [16]. Based on the three-dimensional formulations of the four corresponding problems, we apply the asymptotic expansion method as the thickness of the plate approaches zero, in the case of a homogeneous anisotropic material. Accordingly, we obtain four different two-dimensional plate models: the sensor-actuator model (referring to a plate behaving as a piezoelectric sensor and a piezomagnetic actuator), the actuatorsensor model (referring to a plate behaving as a piezoelectric actuator and a piezomagnetic sensor), the actuator model (according to which the plate behaves as a piezoelectric and piezomagnetic actuator) and the sensor model (according to which the plate behaves as a piezoelectric and piezomagnetic sensor). We validate the asymptotic procedure carried out in each of the four cases by showing weak convergence results. The four two-dimensional plate problems are obtained, as in [14], with different scaling assumptions on the electric and magnetic potentials. On the other hand, they all present common features: for one, the displacement field is always of Kirchhoff-Love type; for two, the temperature variation field is always independent of the thickness coordinate; for three, each problem decouples into a flexural problem - governing the evolution of the transversal displacement of the plate and taking account of an inertia effect involving the mean curvature of the deformed middle surface - and a certain partially or totally coupled membrane problem. In the sensor-actuator model, the membrane problem involves in-plane displacement, temperature variation and electric potential: it is therefore a thermo-piezoelectric problem, the applied magnetic potential playing the role of source term. Since in the actuator-sensor case, the roles of the two potentials are exchanged with respect to the sensor-actuator case, we find a thermo-piezomagnetic membrane problem, the applied electric potential being part of source terms, and we shall not treat this case in detail. In the actuator case, the membrane problem is thermo-elastic, as it just involves in-plane displacement and temperature variation, both the applied electric and magnetic potentials playing the role of source terms. Finally, in the sensor case, the membrane problem is completely coupled, since it involves in-plane displacement, temperature variation, electric potential and magnetic potential; it is then a magneto-electro-thermo-elastic problem. Numerical values of the reduced coefficients can be explicitly computed in each of the four cases; as an example, we report in Table 1 of Appendix 2 the values of such coefficients in the actuator case for the usual (see [2]) $\mathrm{BaTiO}_{3}-\mathrm{CoFe}_{2} \mathrm{O}_{4}$ composite.

\section{Notation}

Scalars are denoted by light-face letters, vector and tensor fields of any order by bold-face letters. We adopt Einstein's usual summation convention; Greek indices take the values 1 and 2, Roman indices range from 1 to 3 . Throughout the paper, $\omega \subset \mathbb{R}^{2}$ denotes a smooth domain in the plane spanned by vectors $\mathbf{e}_{1} \equiv(1,0)$ and $\mathbf{e}_{2} \equiv(0,1)$, with boundary $\gamma$; $\gamma_{0} \subset \gamma$ is a measurable subset of $\gamma$ with strictly positive length measure; $\gamma_{1}:=\gamma \backslash \gamma_{0}$ is the complement of $\gamma_{0}$ with respect to $\gamma$; finally, $0<\varepsilon<1$ is a dimensionless small real parameter which shall tend to zero. For each $\varepsilon$, we define

$$
\begin{array}{ll}
\Omega^{\varepsilon}:=\omega \times\left(-h^{\varepsilon}, h^{\varepsilon}\right), & \Gamma^{\varepsilon}:=\gamma \times\left(-h^{\varepsilon}, h^{\varepsilon}\right) \\
\Gamma_{0}^{\varepsilon}:=\gamma_{0} \times\left(-h^{\varepsilon}, h^{\varepsilon}\right), & \Gamma_{ \pm}^{\varepsilon}:=\omega \times\left\{ \pm h^{\varepsilon}\right\},
\end{array}
$$

with $h^{\varepsilon}>0$. Hence the boundary $\partial \Omega^{\varepsilon}$ of $\Omega^{\varepsilon}$ is partitioned into the lateral face $\Gamma^{\varepsilon}$ and the upper and lower faces $\Gamma_{+}^{\varepsilon}$ and $\Gamma_{-}^{\varepsilon}$; the lateral face is itself partitioned as $\Gamma^{\varepsilon}=\Gamma_{0}^{\varepsilon} \cup \Gamma_{1}^{\varepsilon}$, 
with $\Gamma_{1}^{\varepsilon}:=\gamma_{1} \times\left(-h^{\varepsilon}, h^{\varepsilon}\right)$. Moreover, we let $\widehat{\Gamma}^{\varepsilon}:=\Gamma_{ \pm}^{\varepsilon} \cup \Gamma_{1}^{\varepsilon}=\partial \Omega^{\varepsilon} \backslash \Gamma_{0}^{\varepsilon}$, the complement of $\Gamma_{0}^{\varepsilon}$ with respect to $\partial \Omega^{\varepsilon}$. We set $\mathbf{H}^{1}\left(\Omega^{\varepsilon}\right):=\left[H^{1}\left(\Omega^{\varepsilon}\right)\right]^{3}$ and for $\Xi^{\varepsilon} \subset \partial \Omega^{\varepsilon}$, we define

$$
\begin{aligned}
& H^{1}\left(\Omega^{\varepsilon}, \Xi^{\varepsilon}\right):=\left\{v^{\varepsilon} \in H^{1}\left(\Omega^{\varepsilon}\right) ; \quad v^{\varepsilon}=0 \text { on } \Xi^{\varepsilon}\right\} \\
& \mathbf{H}^{1}\left(\Omega^{\varepsilon}, \Xi^{\varepsilon}\right):=\left\{\mathbf{v}^{\varepsilon}=\left(v_{i}^{\varepsilon}\right) \in \mathbf{H}^{1}\left(\Omega^{\varepsilon}\right) ; \quad \mathbf{v}^{\varepsilon}=\mathbf{0} \text { on } \Xi^{\varepsilon}\right\} .
\end{aligned}
$$

For $\mathbf{v}$ a vector field, $|\mathbf{v}|$ denotes the euclidean norm of $\mathbf{v}$; for $\Phi$ a scalar or vector field, $|\Phi|_{0, \Omega^{\varepsilon}}$ and $\|\Phi\|_{1, \Omega^{\varepsilon}}$ denote, respectively, the $L^{2}\left(\Omega^{\varepsilon}\right)$-norm and the $H^{1}\left(\Omega^{\varepsilon}\right)$-norm of $\Phi$ (analogous notations are used for the $L^{2}\left(\Xi^{\varepsilon}\right)$-norm of $\Phi$ ). At times, for notational convenience, we left tacit the time-dependence of a field $\Phi$. For the sake of brevity, a oneparameter family of fields $\{\Phi(\varepsilon)\}_{\varepsilon>0}$ is referred to as sequence.

\section{Statement of the Problem}

\subsection{Constitutive Laws}

In magneto-electro-thermo-elastic materials the mechanical, electric, magnetic and thermal behaviors are coupled. In the case of the quasi-static approximation for Maxwell's equations, the electric field $\mathbf{E}^{\varepsilon}$ and the magnetic field $\mathbf{H}^{\varepsilon}$ can be expressed through two potential functions, i.e., $E_{i}^{\varepsilon}:=-\partial_{i}^{\varepsilon} \varphi^{\varepsilon}$ and $H_{i}^{\varepsilon}:=-\partial_{i}^{\varepsilon} \zeta^{\varepsilon}$, where $\varphi^{\varepsilon}$ and $\zeta^{\varepsilon}$ denote, respectively, the electric potential and the magnetic potential. Thus, the magneto-electrothermo-elastic state is defined by the quadruplet $\mathcal{U}^{\varepsilon}:=\left(\mathbf{u}^{\varepsilon}, \varphi^{\varepsilon}, \zeta^{\varepsilon}, \theta^{\varepsilon}\right)$ where $\mathbf{u}^{\varepsilon}=\left(u_{i}^{\varepsilon}\right)$ and $\theta^{\varepsilon}$ represent, respectively, the displacement field and the variation of temperature. The interaction between these four different behaviors is described by the following set of constitutive laws:

$$
\left\{\begin{array}{l}
\sigma_{i j}^{\varepsilon}\left(\mathcal{U}^{\varepsilon}\right)=C_{i j k \ell} e_{k \ell}^{\varepsilon}\left(\mathbf{u}^{\varepsilon}\right)+P_{k i j} \partial_{k}^{\varepsilon} \varphi^{\varepsilon}+R_{k i j} \partial_{k}^{\varepsilon} \zeta^{\varepsilon}-\beta_{i j} \theta^{\varepsilon} \\
D_{i}^{\varepsilon}\left(\mathcal{U}^{\varepsilon}\right)=P_{i k \ell} e_{k \ell}^{\varepsilon}\left(\mathbf{u}^{\varepsilon}\right)-X_{i j} \partial_{j}^{\varepsilon} \varphi^{\varepsilon}-\alpha_{i j} \partial_{j}^{\varepsilon} \zeta^{\varepsilon}+p_{i} \theta^{\varepsilon} \\
B_{i}^{\varepsilon}\left(\mathcal{U}^{\varepsilon}\right)=R_{i k \ell} e_{k \ell}^{\varepsilon}\left(\mathbf{u}^{\varepsilon}\right)-\alpha_{i j} \partial_{j}^{\varepsilon} \varphi^{\varepsilon}-M_{i j} \partial_{j}^{\varepsilon} \zeta^{\varepsilon}+m_{i} \theta^{\varepsilon} \\
\mathcal{S}^{\varepsilon}\left(\mathcal{U}^{\varepsilon}\right)=\beta_{i j} e_{i j}^{\varepsilon}\left(\mathbf{u}^{\varepsilon}\right)-p_{i} \partial_{i}^{\varepsilon} \varphi^{\varepsilon}-m_{i} \partial_{i}^{\varepsilon} \zeta^{\varepsilon}+c_{v} \theta^{\varepsilon} \\
q_{i}^{\varepsilon}\left(\theta^{\varepsilon}\right)=-K_{i j} \partial_{j}^{\varepsilon} \theta^{\varepsilon}
\end{array}\right.
$$

where $\boldsymbol{\sigma}^{\varepsilon}=\left(\sigma_{i j}^{\varepsilon}\right)$ is the Cauchy stress tensor, $\mathbf{e}^{\varepsilon}\left(\mathbf{u}^{\varepsilon}\right)=\left(e_{i j}^{\varepsilon}\left(\mathbf{u}^{\varepsilon}\right)\right)$, with $e_{i j}^{\varepsilon}\left(\mathbf{u}^{\varepsilon}\right):=$ $\frac{1}{2}\left(\partial_{i}^{\varepsilon} u_{j}^{\varepsilon}+\partial_{j}^{\varepsilon} u_{i}^{\varepsilon}\right)$, is the linearized strain tensor, $\mathbf{D}^{\varepsilon}=\left(D_{i}^{\varepsilon}\right)$ is the electric displacement field, $\mathbf{B}^{\varepsilon}=\left(B_{i}^{\varepsilon}\right)$ is the magnetic induction field, $\mathcal{S}^{\varepsilon}$ is the thermodynamic entropy and $\mathbf{q}^{\varepsilon}=\left(q_{i}^{\varepsilon}\right)$ is the heat flow vector. $\mathbf{C}=\left(C_{i j k \ell}\right), \mathbf{P}=\left(P_{i j k}\right), \mathbf{R}=\left(R_{i j k}\right), \mathbf{X}=\left(X_{i j}\right)$, $\mathbf{M}=\left(M_{i j}\right), \boldsymbol{\beta}=\left(\beta_{i j}\right), \boldsymbol{\alpha}=\left(\alpha_{i j}\right), \mathbf{p}=\left(p_{i}\right), \mathbf{m}=\left(m_{i}\right), c_{v}$ and $\mathbf{K}=\left(K_{i j}\right)$ represent, respectively, the elasticity tensor, the piezoelectric tensor, the piezomagnetic tensor, the dielectric permittivity tensor, the magnetic permeability tensor, the thermal stress tensor, the magneto-electric tensor, the pyroelectric vector, the pyromagnetic vector, the calorific capacity of the material and the thermal conductivity tensor. Moreover, we suppose the material properties of the (generally anisotropic) magneto-electro-thermo-elastic plate-like body under study to satisfy the usual symmetry, positivity and boundedness conditions, for which we refer to [2]. We recall here the most important hypotheses:

(1) The following symmetric matrix (see [2], [7]) is positive definite

$$
\mathbb{M}^{c}:=\left(\begin{array}{ccc}
\mathbf{X} & \boldsymbol{\alpha} & \mathbf{p} \\
\boldsymbol{\alpha} & \mathbf{M} & \mathbf{m} \\
\mathbf{p}^{T} & \mathbf{m}^{T} & c_{v}
\end{array}\right) .
$$


(2) Without loss of generality we assume that the mass density tensor (see [8], [9])

$$
\boldsymbol{\rho}^{\varepsilon}=\left(\begin{array}{ccc}
\rho_{1}^{\varepsilon} & 0 & 0 \\
0 & \rho_{2}^{\varepsilon} & 0 \\
0 & 0 & \rho_{3}^{\varepsilon}
\end{array}\right)
$$

is diagonal and positive definite ${ }^{\mathrm{a}}$ Hence, $\rho_{i}^{\varepsilon}>0$ and $\rho_{i}^{\varepsilon} \in L^{\infty}\left(\Omega^{\varepsilon}\right)$.

The first hypothesis is verified when the components of the coupling constitutive parameters $\boldsymbol{\alpha}, \mathbf{p}$ and $\mathbf{m}$ are small, which is the case, for instance, for the usual $\mathrm{BaTiO}_{3}-\mathrm{CoFe}_{2} \mathrm{O}_{4}$ composite (see Table 1 in [2]).

\subsection{Governing Equations}

The magneto-electro-thermo-elastic plate is subjected to body forces $\mathbf{f}^{\varepsilon}=\left(f_{i}^{\varepsilon}\right): \Omega^{\varepsilon} \times$ $(0, T) \rightarrow \mathbb{R}^{3}$, an electric charge density $\rho_{e}^{\varepsilon}: \Omega^{\varepsilon} \times(0, T) \rightarrow \mathbb{R}$ and heat source $r^{\varepsilon}: \Omega^{\varepsilon} \times$ $(0, T) \rightarrow \mathbb{R}$. The state $\mathcal{U}^{\varepsilon}$ solves the following system of field equations:

$$
\begin{cases}\boldsymbol{\rho}^{\varepsilon} \ddot{\mathbf{u}}^{\varepsilon}-\operatorname{div}^{\varepsilon} \boldsymbol{\sigma}^{\varepsilon}\left(\mathcal{U}^{\varepsilon}\right)=\mathbf{f}^{\varepsilon} & \text { in } \Omega^{\varepsilon} \times(0, T), \\ \operatorname{div}^{\varepsilon} \mathbf{D}^{\varepsilon}\left(\mathcal{U}^{\varepsilon}\right)=\rho_{e}^{\varepsilon} & \text { in } \Omega^{\varepsilon} \times(0, T), \\ \operatorname{div}^{\varepsilon} \mathbf{B}^{\varepsilon}\left(\mathcal{U}^{\varepsilon}\right)=0 & \text { in } \Omega^{\varepsilon} \times(0, T), \\ \dot{\mathcal{S}}^{\varepsilon}\left(\mathcal{U}^{\varepsilon}\right)+\frac{1}{T_{0}} \operatorname{div}^{\varepsilon} \mathbf{q}^{\varepsilon}\left(\theta^{\varepsilon}\right)=r^{\varepsilon} & \text { in } \Omega^{\varepsilon} \times(0, T),\end{cases}
$$

with $T_{0}>0$ a constant reference temperature. The boundary conditions are posed on $\partial \Omega^{\varepsilon} \times(0, T)$; we recall that $\partial \Omega^{\varepsilon}=\Gamma_{+}^{\varepsilon} \cup \Gamma_{-}^{\varepsilon} \cup \Gamma_{1}^{\varepsilon} \cup \Gamma_{0}^{\varepsilon}$. For simplicity we consider homogeneous boundary conditions on $\Gamma_{0}^{\varepsilon} \times(0, T)$, concerning displacements and temperature, and non-homogeneous boundary conditions on $\widehat{\Gamma}^{\varepsilon} \times(0, T)$, concerning surface forces $\mathbf{g}^{\varepsilon}=\left(g_{i}^{\varepsilon}\right)$ and surface heat flow $\varrho^{\varepsilon}$. Hence, one has

$$
\begin{cases}\boldsymbol{\sigma}^{\varepsilon}\left(\mathcal{U}^{\varepsilon}\right) \mathbf{n}^{\varepsilon}=\mathbf{g}^{\varepsilon} \text { on } \widehat{\Gamma}^{\varepsilon} \times(0, T), & \mathbf{u}^{\varepsilon}=\mathbf{0} \text { on } \Gamma_{0}^{\varepsilon} \times(0, T), \\ \mathbf{q}^{\varepsilon}\left(\theta^{\varepsilon}\right) \cdot \mathbf{n}^{\varepsilon}=\varrho^{\varepsilon} \text { on } \widehat{\Gamma}^{\varepsilon} \times(0, T), & \theta^{\varepsilon}=0 \text { on } \Gamma_{0}^{\varepsilon} \times(0, T) .\end{cases}
$$

As already shown in [14], we specify four possible sets of electromagnetic boundary conditions, leading to four different magneto-electro-thermo-elastic plate models:

$$
\begin{gathered}
(B C)_{1}:\left\{\begin{array}{lll}
\mathbf{D}^{\varepsilon}\left(\mathcal{U}^{\varepsilon}\right) \cdot \mathbf{n}^{\varepsilon}=d^{\varepsilon} \text { on } \widehat{\Gamma}^{\varepsilon} \times(0, T), & \varphi^{\varepsilon}=0 & \text { on } \Gamma_{0}^{\varepsilon} \times(0, T), \\
\mathbf{B}^{\varepsilon}\left(\mathcal{U}^{\varepsilon}\right) \cdot \mathbf{n}^{\varepsilon}=0 & \text { on } \Gamma^{\varepsilon} \times(0, T), & \zeta^{\varepsilon}=\zeta^{ \pm, \varepsilon} \text { on } \Gamma_{ \pm}^{\varepsilon} \times(0, T),
\end{array}\right. \\
(B C)_{2}:\left\{\begin{array}{lll}
\mathbf{D}^{\varepsilon}\left(\mathcal{U}^{\varepsilon}\right) \cdot \mathbf{n}^{\varepsilon}=0 & \text { on } \Gamma^{\varepsilon} \times(0, T), & \varphi^{\varepsilon}=\varphi^{ \pm, \varepsilon} \text { on } \Gamma_{ \pm}^{\varepsilon} \times(0, T), \\
\mathbf{B}^{\varepsilon}\left(\mathcal{U}^{\varepsilon}\right) \cdot \mathbf{n}^{\varepsilon}=b^{\varepsilon} \text { on } \widehat{\Gamma}^{\varepsilon} \times(0, T), & \zeta^{\varepsilon}=0 & \text { on } \Gamma_{0}^{\varepsilon} \times(0, T),
\end{array}\right. \\
(B C)_{3}:\left\{\begin{array}{lll}
\mathbf{D}^{\varepsilon}\left(\mathcal{U}^{\varepsilon}\right) \cdot \mathbf{n}^{\varepsilon}=0 \text { on } \Gamma^{\varepsilon} \times(0, T), & \varphi^{\varepsilon}=\varphi^{ \pm, \varepsilon} \text { on } \Gamma_{ \pm}^{\varepsilon} \times(0, T), \\
\mathbf{B}^{\varepsilon}\left(\mathcal{U}^{\varepsilon}\right) \cdot \mathbf{n}^{\varepsilon}=0 \text { on } \Gamma^{\varepsilon} \times(0, T), & \zeta^{\varepsilon}=\zeta^{ \pm, \varepsilon} & \text { on } \Gamma_{ \pm}^{\varepsilon} \times(0, T),
\end{array}\right. \\
(B C)_{4}: \begin{cases}\mathbf{D}^{\varepsilon}\left(\mathcal{U}^{\varepsilon}\right) \cdot \mathbf{n}^{\varepsilon}=d^{\varepsilon} \text { on } \widehat{\Gamma}^{\varepsilon} \times(0, T), & \varphi^{\varepsilon}=0 \text { on } \Gamma_{0}^{\varepsilon} \times(0, T), \\
\mathbf{B}^{\varepsilon}\left(\mathcal{U}^{\varepsilon}\right) \cdot \mathbf{n}^{\varepsilon}=b^{\varepsilon} \text { on } \widehat{\Gamma}^{\varepsilon} \times(0, T), & \zeta^{\varepsilon}=0 \text { on } \Gamma_{0}^{\varepsilon} \times(0, T),\end{cases}
\end{gathered}
$$

where $\mathbf{n}^{\varepsilon}=\left(n_{i}^{\varepsilon}\right)$ is the outer unit normal vector to $\partial \Omega^{\varepsilon}$. Boundary conditions $(B C)_{1}$ lead to a plate behaving simultaneously as a piezoelectric sensor and a piezomagnetic actuator, namely the sensor-actuator model. Boundary conditions $(B C)_{2}$ lead to a plate

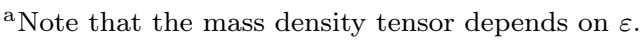


behaving simultaneously as a piezomagnetic sensor and a piezoelectric actuator, namely the actuator-sensor model. Boundary conditions $(B C)_{3}$ are associated with the actuator model, according to which the plate behaves as a piezoeletric and piezomagnetic actuator. Finally, boundary conditions $(B C)_{4}$ are related to the sensor model, whereby the plate behaves as a piezoelectric and piezomagnetic sensor.

The initial conditions are posed in $\Omega^{\varepsilon}$. Let $\mathbf{u}_{0}^{\varepsilon}, \mathbf{u}_{1}^{\varepsilon}, \theta_{0}^{\varepsilon}$ be, respectively, the displacement, the velocity and the temperature at time $t=0$; we have

$$
\left\{\begin{array}{l}
\mathbf{u}^{\varepsilon}\left(x^{\varepsilon}, 0\right)=\mathbf{u}^{\varepsilon}(0)=\mathbf{u}_{0}^{\varepsilon} \text { in } \Omega^{\varepsilon}, \\
\dot{\mathbf{u}}^{\varepsilon}\left(x^{\varepsilon}, 0\right)=\dot{\mathbf{u}}^{\varepsilon}(0)=\mathbf{u}_{1}^{\varepsilon} \text { in } \Omega^{\varepsilon} \\
\theta^{\varepsilon}\left(x^{\varepsilon}, 0\right)=\theta^{\varepsilon}(0)=\theta_{0}^{\varepsilon} \text { in } \Omega^{\varepsilon} .
\end{array}\right.
$$

As pointed out in [2], initial conditions $\varphi_{0}^{\varepsilon}:=\varphi^{\varepsilon}(0)$ and $\zeta_{0}^{\varepsilon}:=\zeta^{\varepsilon}(0)$ are formally given by the solution of the following system of equations:

$$
\left\{\begin{array}{l}
\operatorname{div}^{\varepsilon} \mathbf{D}^{\varepsilon}\left(\mathcal{U}^{\varepsilon}\right)(0)=\partial_{i}^{\varepsilon}\left(P_{i k \ell} e_{k \ell}^{\varepsilon}\left(\mathbf{u}_{0}^{\varepsilon}\right)-X_{i j} \partial_{j}^{\varepsilon} \varphi_{0}^{\varepsilon}-\alpha_{i j} \partial_{j}^{\varepsilon} \zeta_{0}^{\varepsilon}+p_{i} \theta_{0}^{\varepsilon}\right)=\rho_{e}^{\varepsilon} \\
\operatorname{div}^{\varepsilon} \mathbf{B}^{\varepsilon}\left(\mathcal{U}^{\varepsilon}\right)(0)=\partial_{i}^{\varepsilon}\left(R_{i k \ell} e_{k \ell}^{\varepsilon}\left(\mathbf{u}_{0}^{\varepsilon}\right)-\alpha_{i j} \partial_{j}^{\varepsilon} \varphi_{0}^{\varepsilon}-M_{i j} \partial_{j}^{\varepsilon} \zeta_{0}^{\varepsilon}+m_{i} \theta_{0}^{\varepsilon}\right)=0
\end{array}\right.
$$

equipped with suitable boundary conditions.

\subsection{General Weak Formulation}

In order to give a weak formulation of the problems introduced in the previous subsection, we follow Lions [6]. Given a certain state $\mathcal{U}^{\varepsilon}:=\left(\mathbf{u}^{\varepsilon}, \varphi^{\varepsilon}, \zeta^{\varepsilon}, \theta^{\varepsilon}\right)$, for all test functions ${ }^{\mathrm{b}}$ $\mathcal{V}^{\varepsilon}=\left(\mathbf{v}^{\varepsilon}, \psi^{\varepsilon}, \xi^{\varepsilon}, \eta^{\varepsilon}\right)$ and for any fixed $t \in(0, T)$ we introduce the following bilinear form:

$$
\begin{aligned}
A^{\varepsilon}\left(\mathcal{U}^{\varepsilon}(t), \mathcal{V}^{\varepsilon}\right): & =\left(\boldsymbol{\rho}^{\varepsilon} \ddot{\mathbf{u}}^{\varepsilon}, \mathbf{v}^{\varepsilon}\right)+c\left(\eta^{\varepsilon}, \dot{\mathbf{u}}^{\varepsilon}\right)+c_{v}\left(\dot{\theta}^{\varepsilon}, \eta^{\varepsilon}\right)-d\left(\eta^{\varepsilon}, \dot{\varphi}^{\varepsilon}\right)-e\left(\eta^{\varepsilon}, \dot{\zeta}^{\varepsilon}\right)+ \\
& +a_{u}\left(\mathbf{u}^{\varepsilon}, \mathbf{v}^{\varepsilon}\right)+b\left(\varphi^{\varepsilon}, \mathbf{v}^{\varepsilon}\right)-b\left(\psi^{\varepsilon}, \mathbf{u}^{\varepsilon}\right)+f\left(\zeta^{\varepsilon}, \mathbf{v}^{\varepsilon}\right)-f\left(\xi^{\varepsilon}, \mathbf{u}^{\varepsilon}\right)+ \\
& -c\left(\theta^{\varepsilon}, \mathbf{v}^{\varepsilon}\right)+a_{\varphi}\left(\varphi^{\varepsilon}, \psi^{\varepsilon}\right)+a_{\zeta}\left(\zeta^{\varepsilon}, \xi^{\varepsilon}\right)+g\left(\zeta^{\varepsilon}, \psi^{\varepsilon}\right)+g\left(\varphi^{\varepsilon}, \xi^{\varepsilon}\right)+ \\
& -d\left(\theta^{\varepsilon}, \psi^{\varepsilon}\right)-e\left(\theta^{\varepsilon}, \xi^{\varepsilon}\right)+a_{\theta}\left(\theta^{\varepsilon}, \eta^{\varepsilon}\right),
\end{aligned}
$$

where $(\cdot, \cdot)$ is the scalar product in $L^{2}\left(\Omega^{\varepsilon}\right)$ and the bilinear forms $a_{u}(\cdot, \cdot), a_{\varphi}(\cdot, \cdot), a_{\zeta}(\cdot, \cdot)$, $a_{\theta}(\cdot, \cdot), b(\cdot, \cdot), c(\cdot, \cdot), d(\cdot, \cdot), e(\cdot, \cdot), f(\cdot, \cdot), g(\cdot, \cdot)$ are defined as follows:

$$
\begin{array}{rlrl}
a_{u}\left(\mathbf{u}^{\varepsilon}, \mathbf{v}^{\varepsilon}\right) & :=\int_{\Omega^{\varepsilon}} C_{i j k \ell} e_{k \ell}^{\varepsilon}\left(\mathbf{u}^{\varepsilon}\right) e_{i j}^{\varepsilon}\left(\mathbf{v}^{\varepsilon}\right) d x^{\varepsilon}, & a_{\varphi}\left(\varphi^{\varepsilon}, \psi^{\varepsilon}\right) & :=\int_{\Omega^{\varepsilon}} X_{i j} \partial_{j}^{\varepsilon} \varphi^{\varepsilon} \partial_{i}^{\varepsilon} \psi^{\varepsilon} d x^{\varepsilon}, \\
a_{\zeta}\left(\zeta^{\varepsilon}, \xi^{\varepsilon}\right) & :=\int_{\Omega^{\varepsilon}} M_{i j} \partial_{j}^{\varepsilon} \zeta^{\varepsilon} \partial_{i}^{\varepsilon} \xi^{\varepsilon} d x^{\varepsilon}, & & a_{\theta}\left(\theta^{\varepsilon}, \eta^{\varepsilon}\right):=\frac{1}{T_{0}} \int_{\Omega^{\varepsilon}} K_{i j} \partial_{j}^{\varepsilon} \theta^{\varepsilon} \partial_{i}^{\varepsilon} \eta^{\varepsilon} d x^{\varepsilon}, \\
b\left(\psi^{\varepsilon}, \mathbf{u}^{\varepsilon}\right):=\int_{\Omega^{\varepsilon}} P_{k i j} \partial_{k}^{\varepsilon} \psi^{\varepsilon} e_{i j}^{\varepsilon}\left(\mathbf{u}^{\varepsilon}\right) d x^{\varepsilon}, & c\left(\eta^{\varepsilon}, \mathbf{u}^{\varepsilon}\right):=\int_{\Omega^{\varepsilon}} \eta^{\varepsilon} \beta_{i j} e_{i j}^{\varepsilon}\left(\mathbf{u}^{\varepsilon}\right) d x^{\varepsilon}, \\
d\left(\eta^{\varepsilon}, \varphi^{\varepsilon}\right):=\int_{\Omega^{\varepsilon}} \eta^{\varepsilon} p_{k} \partial_{k}^{\varepsilon} \varphi^{\varepsilon} d x^{\varepsilon}, & e\left(\eta^{\varepsilon}, \zeta^{\varepsilon}\right):=\int_{\Omega^{\varepsilon}} \eta^{\varepsilon} m_{k} \partial_{k}^{\varepsilon} \zeta^{\varepsilon} d x^{\varepsilon}, \\
f\left(\xi^{\varepsilon}, \mathbf{u}^{\varepsilon}\right):=\int_{\Omega^{\varepsilon}} R_{k i j} \partial_{k}^{\varepsilon} \xi^{\varepsilon} e_{i j}^{\varepsilon}\left(\mathbf{u}^{\varepsilon}\right) d x^{\varepsilon}, & g\left(\zeta^{\varepsilon}, \psi^{\varepsilon}\right):=\int_{\Omega^{\varepsilon}} \alpha_{i j} \partial_{j}^{\varepsilon} \zeta^{\varepsilon} \partial_{i}^{\varepsilon} \psi^{\varepsilon} d x^{\varepsilon} .
\end{array}
$$

For a given state $\mathcal{U}^{\varepsilon}:=\left(\mathbf{u}^{\varepsilon}, \varphi^{\varepsilon}, \zeta^{\varepsilon}, \theta^{\varepsilon}\right)$, the energy functional of the system is:

$$
\begin{aligned}
\mathcal{E}^{\varepsilon}(t):= & \frac{1}{2}\left\{\left(\boldsymbol{\rho}^{\varepsilon} \dot{\mathbf{u}}^{\varepsilon}, \dot{\mathbf{u}}^{\varepsilon}\right)+a_{u}\left(\mathbf{u}^{\varepsilon}, \mathbf{u}^{\varepsilon}\right)+a_{\varphi}\left(\varphi^{\varepsilon}, \varphi^{\varepsilon}\right)+a_{\zeta}\left(\zeta^{\varepsilon}, \zeta^{\varepsilon}\right)+\left(c_{v}^{\varepsilon} \theta^{\varepsilon}, \theta^{\varepsilon}\right)+\right. \\
& \left.-2 d\left(\theta^{\varepsilon}, \varphi^{\varepsilon}\right)-2 e\left(\theta^{\varepsilon}, \zeta^{\varepsilon}\right)+2 g\left(\zeta^{\varepsilon}, \varphi^{\varepsilon}\right)\right\} .
\end{aligned}
$$

${ }^{\mathrm{b}}$ The space of test functions shall be precised case by case, according to the specific problem under study. 
In the sequel we shall distinguish among the four variational evolution problems arising from the different possible boundary conditions presented in the previous subsection.

1) The sensor-actuator model. We let $\bar{\zeta}^{\varepsilon}:=\zeta^{\varepsilon}-\widehat{\zeta}^{\varepsilon}$, where $\widehat{\zeta}^{\varepsilon}$ is a trace lifting in $H^{1}\left(\Omega^{\varepsilon}\right)$ of the magnetic boundary potentials $\zeta^{ \pm, \varepsilon}$ acting on $\Gamma_{ \pm}^{\varepsilon}$. The weak formulation of (1.2)-(1.3) with electromagnetic boundary conditions $(B C)_{1}$ takes the following form

Find $\mathcal{U}^{\varepsilon}=\left(\mathbf{u}^{\varepsilon}, \varphi^{\varepsilon}, \bar{\zeta}^{\varepsilon}, \theta^{\varepsilon}\right) \in \mathbf{H}^{1}\left(\Omega^{\varepsilon}, \Gamma_{0}^{\varepsilon}\right) \times H^{1}\left(\Omega^{\varepsilon}, \Gamma_{0}^{\varepsilon}\right) \times H^{1}\left(\Omega^{\varepsilon}, \Gamma_{ \pm}^{\varepsilon}\right) \times H^{1}\left(\Omega^{\varepsilon}, \Gamma_{0}^{\varepsilon}\right)$,

such that, for all $\mathcal{V}^{\varepsilon} \in \mathbf{H}^{1}\left(\Omega^{\varepsilon}, \Gamma_{0}^{\varepsilon}\right) \times H^{1}\left(\Omega^{\varepsilon}, \Gamma_{0}^{\varepsilon}\right) \times H^{1}\left(\Omega^{\varepsilon}, \Gamma_{ \pm}^{\varepsilon}\right) \times H^{1}\left(\Omega^{\varepsilon}, \Gamma_{0}^{\varepsilon}\right)$,

$A^{\varepsilon}\left(\mathcal{U}^{\varepsilon}(t), \mathcal{V}^{\varepsilon}\right)=L_{1}^{\varepsilon}\left(\mathcal{V}^{\varepsilon}\right), t \in(0, T)$

with initial conditions $\left(\mathbf{u}_{0}^{\varepsilon}, \mathbf{u}_{1}^{\varepsilon}, \theta_{0}^{\varepsilon}\right)$ and

$$
\begin{aligned}
L_{1}^{\varepsilon}\left(\mathcal{V}^{\varepsilon}\right):= & \left(\mathbf{f}^{\varepsilon}, \mathbf{v}^{\varepsilon}\right)+\left(\mathbf{g}^{\varepsilon}, \mathbf{v}^{\varepsilon}\right)_{\mathbf{L}^{2}\left(\widehat{\Gamma}^{\varepsilon}\right)}+\left(r^{\varepsilon}, \eta^{\varepsilon}\right)-\left(\varrho^{\varepsilon}, \eta^{\varepsilon}\right){ }_{L^{2}\left(\widehat{\Gamma}^{\varepsilon}\right)}+\left(\rho_{e}^{\varepsilon}, \psi^{\varepsilon}\right)+ \\
& -\left(d^{\varepsilon}, \psi^{\varepsilon}\right)_{L^{2}\left(\widehat{\Gamma}^{\varepsilon}\right)}-a_{\zeta}\left(\widehat{\zeta}^{\varepsilon}, \xi^{\varepsilon}\right)-f\left(\widehat{\zeta}^{\varepsilon}, \mathbf{v}^{\varepsilon}\right)+e\left(\eta^{\varepsilon}, \dot{\dot{\zeta}^{\varepsilon}}\right)-g\left(\widehat{\zeta}^{\varepsilon}, \psi^{\varepsilon}\right),
\end{aligned}
$$

For all weak solutions $\mathcal{U}^{\varepsilon}=\left(\mathbf{u}^{\varepsilon}, \varphi^{\varepsilon}, \bar{\zeta}^{\varepsilon}, \theta^{\varepsilon}\right)$ of (1.11), the energy (1.10) satisfies (see [2]):

$$
\dot{\mathcal{E}}^{\varepsilon}(t)+a_{\theta}\left(\theta^{\varepsilon}(t), \theta^{\varepsilon}(t)\right)=L_{\mathcal{E}}^{1}(t)
$$

with $L_{\mathcal{E}}^{1}(t):=\left(\mathbf{f}^{\varepsilon}, \dot{\mathbf{u}}^{\varepsilon}\right)+\left(\mathbf{g}^{\varepsilon}, \dot{\mathbf{u}}^{\varepsilon}\right)_{\mathbf{L}^{2}\left(\widehat{\Gamma}^{\varepsilon}\right)}+\left(\dot{\rho}_{e}^{\varepsilon}, \varphi^{\varepsilon}\right)-\left(\dot{d}^{\varepsilon}, \varphi^{\varepsilon}\right)_{L^{2}\left(\widehat{\Gamma}^{\varepsilon}\right)}+\left(r^{\varepsilon}, \theta^{\varepsilon}\right)-\left(\varrho^{\varepsilon}, \theta^{\varepsilon}\right)_{L^{2}\left(\widehat{\Gamma}^{\varepsilon}\right)}-$ $a_{\zeta}\left(\dot{\dot{\zeta}^{\varepsilon}}, \bar{\zeta}^{\varepsilon}\right)-f\left(\widehat{\zeta}^{\varepsilon}, \dot{\mathbf{u}}^{\varepsilon}\right)+e\left(\theta^{\varepsilon}, \dot{\widehat{\zeta}}^{\varepsilon}\right)-g\left(\dot{\hat{\zeta}^{\varepsilon}}, \varphi^{\varepsilon}\right)$.

2) The actuator-sensor model. We let $\bar{\varphi}^{\varepsilon}:=\varphi^{\varepsilon}-\widehat{\varphi}^{\varepsilon}$, where $\widehat{\varphi}^{\varepsilon}$ is a trace lifting in $H^{1}\left(\Omega^{\varepsilon}\right)$ of the electric boundary potentials $\varphi^{ \pm, \varepsilon}$ acting on $\Gamma_{ \pm}^{\varepsilon}$. The weak formulation of (1.2)-(1.3) with electromagnetic boundary conditions $(B C)_{2}$ takes the following form

Find $\mathcal{U}^{\varepsilon}=\left(\mathbf{u}^{\varepsilon}, \bar{\varphi}^{\varepsilon}, \zeta^{\varepsilon}, \theta^{\varepsilon}\right) \in \mathbf{H}^{1}\left(\Omega^{\varepsilon}, \Gamma_{0}^{\varepsilon}\right) \times H^{1}\left(\Omega^{\varepsilon}, \Gamma_{ \pm}^{\varepsilon}\right) \times H^{1}\left(\Omega^{\varepsilon}, \Gamma_{0}^{\varepsilon}\right) \times H^{1}\left(\Omega^{\varepsilon}, \Gamma_{0}^{\varepsilon}\right)$,

such that, for all $\mathcal{V}^{\varepsilon} \in \mathbf{H}^{1}\left(\Omega^{\varepsilon}, \Gamma_{0}^{\varepsilon}\right) \times H^{1}\left(\Omega^{\varepsilon}, \Gamma_{ \pm}^{\varepsilon}\right) \times H^{1}\left(\Omega^{\varepsilon}, \Gamma_{0}^{\varepsilon}\right) \times H^{1}\left(\Omega^{\varepsilon}, \Gamma_{0}^{\varepsilon}\right)$,

$A^{\varepsilon}\left(\mathcal{U}^{\varepsilon}(t), \mathcal{V}^{\varepsilon}\right)=L_{2}^{\varepsilon}\left(\mathcal{V}^{\varepsilon}\right), t \in(0, T)$

with initial conditions $\left(\mathbf{u}_{0}^{\varepsilon}, \mathbf{u}_{1}^{\varepsilon}, \theta_{0}^{\varepsilon}\right)$ and

$$
\begin{aligned}
& L_{2}^{\varepsilon}\left(\mathcal{V}^{\varepsilon}\right):=\left(\mathbf{f}^{\varepsilon}, \mathbf{v}^{\varepsilon}\right)+\left(\mathbf{g}^{\varepsilon}, \mathbf{v}^{\varepsilon}\right)_{\mathbf{L}^{2}\left(\widehat{\Gamma}^{\varepsilon}\right)}+\left(r^{\varepsilon}, \eta^{\varepsilon}\right)-\left(\varrho^{\varepsilon}, \eta^{\varepsilon}\right)_{L^{2}\left(\widehat{\Gamma}^{\varepsilon}\right)}+\left(\rho_{e}^{\varepsilon}, \psi^{\varepsilon}\right)+ \\
&-\left(b^{\varepsilon}, \xi^{\varepsilon}\right) L^{2}\left(\widehat{\Gamma}^{\varepsilon}\right) \\
&-a_{\varphi}\left(\widehat{\varphi}^{\varepsilon}, \psi^{\varepsilon}\right)-b\left(\widehat{\varphi}^{\varepsilon}, \mathbf{v}^{\varepsilon}\right)+d\left(\eta^{\varepsilon}, \dot{\widehat{\varphi}}^{\varepsilon}\right)-g\left(\xi^{\varepsilon}, \widehat{\varphi}^{\varepsilon}\right),
\end{aligned}
$$

For all weak solutions $\mathcal{U}^{\varepsilon}=\left(\mathbf{u}^{\varepsilon}, \varphi^{\varepsilon}, \bar{\zeta}^{\varepsilon}, \theta^{\varepsilon}\right)$ of (1.12), the energy (1.10) satisfies :

$$
\dot{\mathcal{E}}^{\varepsilon}(t)+a_{\theta}\left(\theta^{\varepsilon}(t), \theta^{\varepsilon}(t)\right)=L_{\mathcal{E}}^{2}(t)
$$

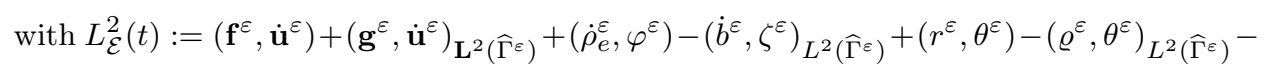
$a_{\varphi}\left(\dot{\widehat{\varphi}}^{\varepsilon}, \bar{\varphi}^{\varepsilon}\right)-b\left(\widehat{\varphi}^{\varepsilon}, \dot{\mathbf{u}}^{\varepsilon}\right)+d\left(\theta^{\varepsilon}, \dot{\widehat{\varphi}}^{\varepsilon}\right)-g\left(\zeta^{\varepsilon}, \dot{\widehat{\varphi}}^{\varepsilon}\right)$.

3) The actuator model. The weak formulation of (1.2)-(1.3) with electromagnetic boundary conditions $(B C)_{3}$ takes the following form

$$
\left\{\begin{array}{l}
\text { Find } \mathcal{U}^{\varepsilon}=\left(\mathbf{u}^{\varepsilon}, \bar{\varphi}^{\varepsilon}, \bar{\zeta}^{\varepsilon}, \theta^{\varepsilon}\right) \in \mathbf{H}^{1}\left(\Omega^{\varepsilon}, \Gamma_{0}^{\varepsilon}\right) \times H^{1}\left(\Omega^{\varepsilon}, \Gamma_{ \pm}^{\varepsilon}\right) \times H^{1}\left(\Omega^{\varepsilon}, \Gamma_{ \pm}^{\varepsilon}\right) \times H^{1}\left(\Omega^{\varepsilon}, \Gamma_{0}^{\varepsilon}\right), \\
\text { such that, for all } \mathcal{V}^{\varepsilon} \in \mathbf{H}^{1}\left(\Omega^{\varepsilon}, \Gamma_{0}^{\varepsilon}\right) \times H^{1}\left(\Omega^{\varepsilon}, \Gamma_{ \pm}^{\varepsilon}\right) \times H^{1}\left(\Omega^{\varepsilon}, \Gamma_{ \pm}^{\varepsilon}\right) \times H^{1}\left(\Omega^{\varepsilon}, \Gamma_{0}^{\varepsilon}\right), \\
A^{\varepsilon}\left(\mathcal{U}^{\varepsilon}(t), \mathcal{V}^{\varepsilon}\right)=L_{3}^{\varepsilon}\left(\mathcal{V}^{\varepsilon}\right), t \in(0, T)
\end{array}\right.
$$


with initial conditions $\left(\mathbf{u}_{0}^{\varepsilon}, \mathbf{u}_{1}^{\varepsilon}, \theta_{0}^{\varepsilon}\right)$ and

$$
\begin{aligned}
& L_{3}^{\varepsilon}\left(\mathcal{V}^{\varepsilon}\right):=\left(\mathbf{f}^{\varepsilon}, \mathbf{v}^{\varepsilon}\right)+\left(\mathbf{g}^{\varepsilon}, \mathbf{v}^{\varepsilon}\right) \mathbf{L}^{2}\left(\widehat{\Gamma}^{\varepsilon}\right)+\left(r^{\varepsilon}, \eta^{\varepsilon}\right)-\left(\varrho^{\varepsilon}, \eta^{\varepsilon}\right) L^{2}\left(\widehat{\Gamma}^{\varepsilon}\right) \\
&-a_{\zeta}\left(\widehat{\zeta}^{\varepsilon}, \psi^{\varepsilon}\right)-f\left(\rho_{e}^{\varepsilon}, \psi^{\varepsilon}\right)+ \\
&-a_{\varphi}\left(\widehat{\varphi}^{\varepsilon}, \mathbf{v}^{\varepsilon}\right)+e\left(\eta^{\varepsilon}, \dot{\hat{\zeta}^{\varepsilon}}\right)-g\left(\widehat{\bar{\zeta}}^{\varepsilon}, \mathbf{v}^{\varepsilon}\right)+d\left(\eta^{\varepsilon}, \psi^{\varepsilon}\right)+ \\
&\left.\dot{\varphi}^{\varepsilon}\right)-g\left(\xi^{\varepsilon}, \widehat{\varphi}^{\varepsilon}\right) .
\end{aligned}
$$

For all weak solutions $\mathcal{U}^{\varepsilon}=\left(\mathbf{u}^{\varepsilon}, \varphi^{\varepsilon}, \bar{\zeta}^{\varepsilon}, \theta^{\varepsilon}\right)$ of (1.13), the energy (1.10) satisfies :

$$
\dot{\mathcal{E}}^{\varepsilon}(t)+a_{\theta}\left(\theta^{\varepsilon}(t), \theta^{\varepsilon}(t)\right)=L_{\mathcal{E}}^{3}(t),
$$

with $L_{\mathcal{E}}^{3}(t):=\left(\mathbf{f}^{\varepsilon}, \dot{\mathbf{u}}^{\varepsilon}\right)+\left(\mathbf{g}^{\varepsilon}, \dot{\mathbf{u}}^{\varepsilon}\right)_{\mathbf{L}^{2}\left(\widehat{\Gamma}^{\varepsilon}\right)}+\left(\dot{\rho}_{e}^{\varepsilon}, \varphi^{\varepsilon}\right)+\left(r^{\varepsilon}, \theta^{\varepsilon}\right)-\left(\varrho^{\varepsilon}, \theta^{\varepsilon}\right)_{L^{2}\left(\widehat{\Gamma}^{\varepsilon}\right)}-a_{\zeta}\left(\dot{\widehat{\zeta}}^{\varepsilon}, \bar{\zeta}^{\varepsilon}\right)-$ $f\left(\widehat{\zeta}^{\varepsilon}, \dot{\mathbf{u}}^{\varepsilon}\right)+e\left(\theta^{\varepsilon}, \dot{\hat{\zeta}^{\varepsilon}}\right)-g\left(\dot{\hat{\zeta}^{\varepsilon}}, \varphi^{\varepsilon}\right)-a_{\varphi}\left(\dot{\hat{\varphi}^{\varepsilon}}, \bar{\varphi}^{\varepsilon}\right)-b\left(\widehat{\varphi}^{\varepsilon}, \dot{\mathbf{u}}^{\varepsilon}\right)+d\left(\theta^{\varepsilon}, \dot{\hat{\varphi}^{\varepsilon}}\right)-g\left(\zeta^{\varepsilon}, \dot{\hat{\varphi}}^{\varepsilon}\right)$.

4) The sensor model. The weak formulation of (1.2)-(1.3) with electromagnetic boundary conditions $(B C)_{4}$ takes the following form

$$
\left\{\begin{array}{l}
\text { Find } \mathcal{U}^{\varepsilon}=\left(\mathbf{u}^{\varepsilon}, \varphi^{\varepsilon}, \zeta^{\varepsilon}, \theta^{\varepsilon}\right) \in \mathbf{H}^{1}\left(\Omega^{\varepsilon}, \Gamma_{0}^{\varepsilon}\right) \times H^{1}\left(\Omega^{\varepsilon}, \Gamma_{0}^{\varepsilon}\right) \times H^{1}\left(\Omega^{\varepsilon}, \Gamma_{0}^{\varepsilon}\right) \times H^{1}\left(\Omega^{\varepsilon}, \Gamma_{0}^{\varepsilon}\right), \\
\text { such that, for all } \mathcal{V}^{\varepsilon} \in \mathbf{H}^{1}\left(\Omega^{\varepsilon}, \Gamma_{0}^{\varepsilon}\right) \times H^{1}\left(\Omega^{\varepsilon}, \Gamma_{0}^{\varepsilon}\right) \times H^{1}\left(\Omega^{\varepsilon}, \Gamma_{0}^{\varepsilon}\right) \times H^{1}\left(\Omega^{\varepsilon}, \Gamma_{0}^{\varepsilon}\right), \\
A^{\varepsilon}\left(\mathcal{U}^{\varepsilon}(t), \mathcal{V}^{\varepsilon}\right)=L_{4}^{\varepsilon}\left(\mathcal{V}^{\varepsilon}\right), t \in(0, T)
\end{array}\right.
$$

with initial conditions $\left(\mathbf{u}_{0}^{\varepsilon}, \mathbf{u}_{1}^{\varepsilon}, \theta_{0}^{\varepsilon}\right)$ and

$$
\begin{aligned}
L_{4}^{\varepsilon}\left(\mathcal{V}^{\varepsilon}\right):= & \left(\mathbf{f}^{\varepsilon}, \mathbf{v}^{\varepsilon}\right)+\left(\mathbf{g}^{\varepsilon}, \mathbf{v}^{\varepsilon}\right) \\
& \left.-\left(b^{\varepsilon}, \xi^{\varepsilon}\right)_{L^{2}\left(\widehat{\Gamma}^{\varepsilon}\right)}+\left(d^{\varepsilon}, \psi^{\varepsilon}\right){ }_{L^{2}\left(r^{\varepsilon},\right.}, \eta^{\varepsilon}\right)-\left(\varrho^{\varepsilon}, \eta^{\varepsilon}\right)
\end{aligned}
$$

For all weak solutions $\mathcal{U}^{\varepsilon}=\left(\mathbf{u}^{\varepsilon}, \varphi^{\varepsilon}, \bar{\zeta}^{\varepsilon}, \theta^{\varepsilon}\right)$ of (1.14), the energy (1.10) satisfies :

$$
\dot{\mathcal{E}}^{\varepsilon}(t)+a_{\theta}\left(\theta^{\varepsilon}(t), \theta^{\varepsilon}(t)\right)=L_{\mathcal{E}}^{4}(t),
$$

with $L_{\mathcal{E}}^{4}(t):=\left(\mathbf{f}^{\varepsilon}, \dot{\mathbf{u}}^{\varepsilon}\right)+\left(\mathbf{g}^{\varepsilon}, \dot{\mathbf{u}}^{\varepsilon}\right)_{\mathbf{L}^{2}\left(\widehat{\Gamma}^{\varepsilon}\right)}+\left(\dot{\rho}_{e}^{\varepsilon}, \varphi^{\varepsilon}\right)-\left(\dot{b}^{\varepsilon}, \zeta^{\varepsilon}\right)_{L^{2}\left(\widehat{\Gamma}^{\varepsilon}\right)}-\left(\dot{d}^{\varepsilon}, \varphi^{\varepsilon}\right)_{L^{2}\left(\widehat{\Gamma}^{\varepsilon}\right)}+\left(r^{\varepsilon}, \theta^{\varepsilon}\right)-$ $\left(\varrho^{\varepsilon}, \theta^{\varepsilon}\right)_{L^{2}\left(\widehat{\Gamma}^{\varepsilon}\right)}$.

Finally (see 1.9), initial conditions $\varphi_{0}^{\varepsilon}$ and $\bar{\zeta}_{0}^{\varepsilon}$ are given by the solution of a suitable variational problem, according to the case under study; for instance, in the sensor-actuator case, we have

$$
\left\{\begin{array}{l}
\text { Find }\left(\varphi_{0}^{\varepsilon}, \bar{\zeta}_{0}^{\varepsilon}\right) \in H^{1}\left(\Omega^{\varepsilon}, \Gamma_{0}^{\varepsilon}\right) \times H^{1}\left(\Omega^{\varepsilon}, \Gamma_{ \pm}^{\varepsilon}\right) \text { such that } \\
\text { for all }\left(\psi^{\varepsilon}, \xi^{\varepsilon}\right) \in H^{1}\left(\Omega^{\varepsilon}, \Gamma_{0}^{\varepsilon}\right) \times H^{1}\left(\Omega^{\varepsilon}, \Gamma_{ \pm}^{\varepsilon}\right) \\
a_{\varphi}\left(\varphi_{0}^{\varepsilon}, \psi^{\varepsilon}\right)+a_{\zeta}\left(\bar{\zeta}_{0}^{\varepsilon}, \xi^{\varepsilon}\right)+g\left(\bar{\zeta}_{0}^{\varepsilon}, \psi^{\varepsilon}\right)+g\left(\varphi_{0}^{\varepsilon}, \xi^{\varepsilon}\right)=\left(\rho_{e}^{\varepsilon}(0), \psi^{\varepsilon}\right)+ \\
-\left(d^{\varepsilon}(0), \psi^{\varepsilon}\right)_{L^{2}\left(\widehat{\Gamma}^{\varepsilon}\right)}-a_{\zeta}\left(\widehat{\zeta}_{0}^{\varepsilon}, \psi^{\varepsilon}\right)+f\left(\xi^{\varepsilon}, \mathbf{u}_{0}^{\varepsilon}\right)-e\left(\theta_{0}^{\varepsilon}, \xi^{\varepsilon}\right)-g\left(\widehat{\zeta}_{0}^{\varepsilon}, \psi^{\varepsilon}\right)+d\left(\theta_{0}^{\varepsilon}, \psi^{\varepsilon}\right)
\end{array}\right.
$$

\subsection{Existence, Uniqueness and Regularity}

We state here a result of well-posedness for the problem in the sensor-actuator case, the other cases being analogous. Let us explicitly remark that the assumptions on initial conditions, source and boundary values are stronger than those used in [2].

Theorem 1.1. Suppose $\Omega^{\varepsilon}$ is a convex domain with Lipschitz-continuous boundary. Suppose the initial data are such that: 
(i) $\mathbf{u}_{0}^{\varepsilon} \in \mathbf{H}^{2}\left(\Omega^{\varepsilon}\right) \cap \mathbf{H}^{1}\left(\Omega^{\varepsilon}, \Gamma_{0}^{\varepsilon}\right), \mathbf{u}_{1}^{\varepsilon} \in \mathbf{H}^{1}\left(\Omega^{\varepsilon}, \Gamma_{0}^{\varepsilon}\right), \theta_{0}^{\varepsilon} \in H^{2}\left(\Omega^{\varepsilon}\right) \cap H^{1}\left(\Omega^{\varepsilon}, \Gamma_{0}^{\varepsilon}\right)$,

(ii) $\quad \mathbf{u}_{0}^{\varepsilon}$ and $\theta_{0}^{\varepsilon}$ are such that problem (1.15) admits a solution $\left(\varphi_{0}^{\varepsilon}, \bar{\zeta}_{0}^{\varepsilon}\right) \in H^{2}\left(\Omega^{\varepsilon}\right) \cap H^{1}\left(\Omega^{\varepsilon}, \Gamma_{0}^{\varepsilon}\right) \times H^{2}\left(\Omega^{\varepsilon}\right) \cap H^{1}\left(\Omega^{\varepsilon}, \Gamma_{ \pm}^{\varepsilon}\right)$.

Secondly, assume the following regularity properties on the source and boundary values:

$$
\left\{\begin{array}{l}
\mathbf{f}^{\varepsilon} \in H^{1}\left(0, T ; \mathbf{L}^{2}\left(\Omega^{\varepsilon}\right)\right) \cap C^{0}\left([0, T] ; \mathbf{L}^{2}\left(\Omega^{\varepsilon}\right)\right), \\
\rho_{e} \in H^{2}\left(0, T ; L^{2}\left(\Omega^{\varepsilon}\right)\right) \cap C^{1}\left([0, T] ; L^{2}\left(\Omega^{\varepsilon}\right)\right), \\
r \in H^{1}\left(0, T ; L^{2}\left(\Omega^{\varepsilon}\right)\right) \cap C^{0}\left([0, T] ; L^{2}\left(\Omega^{\varepsilon}\right)\right), \\
\mathbf{g}^{\varepsilon} \in H^{2}\left(0, T ; \mathbf{L}^{2}\left(\widehat{\Gamma}^{\varepsilon}\right)\right) \cap C^{1}\left([0, T] ; \mathbf{L}^{2}\left(\widehat{\Gamma}^{\varepsilon}\right)\right), \\
d^{\varepsilon} \in H^{2}\left(0, T ; L^{2}\left(\widehat{\Gamma}^{\varepsilon}\right)\right) \cap C^{1}\left([0, T] ; L^{2}\left(\widehat{\Gamma}^{\varepsilon}\right)\right), \\
b^{\varepsilon} \in H^{2}\left(0, T ; L^{2}\left(\Gamma^{\varepsilon}\right)\right) \cap C^{1}\left([0, T] ; L^{2}\left(\Gamma^{\varepsilon}\right)\right), \\
\varrho^{\varepsilon} \in H^{1}\left(0, T ; L^{2}\left(\widehat{\Gamma}^{\varepsilon}\right)\right) \cap C^{0}\left([0, T] ; L^{2}\left(\widehat{\Gamma}^{\varepsilon}\right)\right),
\end{array}\right.
$$

and on the constitutive parameters:

$$
C_{i j k \ell}, P_{i j k}, R_{i j k}, \beta_{i j}, K_{i j} \in W^{1, \infty}\left(\Omega^{\varepsilon}\right)
$$

Also, let the following compatibility conditions be satisfied:

$$
\begin{cases}\mathbf{g}^{\varepsilon}(0)=\boldsymbol{\sigma}\left(\mathbf{u}_{0}^{\varepsilon}, \varphi_{0}^{\varepsilon}, \zeta_{0}^{\varepsilon}, \theta_{0}^{\varepsilon}\right) \mathbf{n}^{\varepsilon} & \text { on } \widehat{\Gamma}^{\varepsilon}, \\ d^{\varepsilon}(0)=\mathbf{D}^{\varepsilon}\left(\mathbf{u}_{0}^{\varepsilon}, \varphi_{0}^{\varepsilon}, \zeta_{0}^{\varepsilon}, \theta_{0}^{\varepsilon}\right) \cdot \mathbf{n}^{\varepsilon} & \text { on } \widehat{\Gamma}^{\varepsilon}, \\ b^{\varepsilon}(0)=\mathbf{B}^{\varepsilon}\left(\mathbf{u}_{0}^{\varepsilon}, \varphi_{0}^{\varepsilon}, \zeta_{0}^{\varepsilon}, \theta_{0}^{\varepsilon}\right) \cdot \mathbf{n}^{\varepsilon} & \text { on } \Gamma^{\varepsilon}, \\ \varrho^{\varepsilon}(0)=\mathbf{q}^{\varepsilon}\left(\theta_{0}^{\varepsilon}\right) \cdot \mathbf{n}^{\varepsilon} & \text { on } \widehat{\Gamma}^{\varepsilon} .\end{cases}
$$

Then, problem (1.11) admits a unique solution $\left(\mathbf{u}^{\varepsilon}, \varphi^{\varepsilon}, \bar{\zeta}^{\varepsilon}, \theta^{\varepsilon}\right)$ such that

$$
\left\{\begin{array}{l}
\mathbf{u}^{\varepsilon} \in C^{0}\left([0, T] ; \mathbf{H}^{1}\left(\Omega^{\varepsilon}, \Gamma_{0}^{\varepsilon}\right)\right) \cap C^{1}\left([0, T] ; \mathbf{L}^{2}\left(\Omega^{\varepsilon}\right)\right), \\
\dot{\mathbf{u}}^{\varepsilon} \in L^{2}\left(0, T ; \mathbf{H}^{1}\left(\Omega^{\varepsilon}, \Gamma_{0}^{\varepsilon}\right)\right), \\
\ddot{\mathbf{u}}^{\varepsilon} \in L^{2}\left(0, T ; \mathbf{L}^{2}\left(\Omega^{\varepsilon}\right)\right), \\
\varphi^{\varepsilon} \in H^{1}\left(0, T ; H^{1}\left(\Omega^{\varepsilon}, \Gamma_{0}^{\varepsilon}\right)\right) \cap C^{0}\left([0, T] ; H^{1}\left(\Omega^{\varepsilon}, \Gamma_{0}^{\varepsilon}\right)\right), \\
\bar{\zeta}^{\varepsilon} \in H^{1}\left(0, T ; H^{1}\left(\Omega^{\varepsilon}, \Gamma_{ \pm}^{\varepsilon}\right)\right) \cap C^{0}\left([0, T] ; H^{1}\left(\Omega^{\varepsilon}, \Gamma_{ \pm}^{\varepsilon}\right)\right), \\
\theta^{\varepsilon} \in H^{1}\left(0, T ; H^{1}\left(\Omega^{\varepsilon}, \Gamma_{0}^{\varepsilon}\right)\right) \cap C^{0}\left([0, T] ; H^{1}\left(\Omega^{\varepsilon}, \Gamma_{0}^{\varepsilon}\right)\right) .
\end{array}\right.
$$

Let us explicitly remark that the convexity hypothesis for the domain ensures condition (ii) to be automatically satisfied (see, e.g., [4]).

\section{Scaled Evolution Problems}

In order to perform an asymptotic analysis, we need to transform problems (1.11), (1.12), (1.13), (1.14), posed on a variable domain $\Omega^{\varepsilon}$, onto problems posed on a fixed domain $\Omega$ (independent of $\varepsilon$ ). We suppose the thickness of the plate $h^{\varepsilon}$ to depend linearly on $\varepsilon$, so that $h^{\varepsilon}=\varepsilon h$. We apply the usual change of variables (see [3]) and drop the index $\varepsilon$ from all the subsets of the fixed domain and the fields therein defined. Let us suppose that the data verify the following scaling assumptions:

$$
\begin{array}{lll}
f_{\alpha}^{\varepsilon}\left(x^{\varepsilon}, t\right)=f_{\alpha}(x, t), & f_{3}^{\varepsilon}\left(x^{\varepsilon}, t\right)=\varepsilon f_{3}(x, t), & x \in \Omega, \\
g_{\alpha}^{\varepsilon}\left(x^{\varepsilon}, t\right)=g_{\alpha}(x, t), & g_{3}^{\varepsilon}\left(x^{\varepsilon}, t\right)=\varepsilon g_{3}(x, t), & x \in \Gamma_{1}, \\
g_{\alpha}^{\varepsilon}\left(x^{\varepsilon}, t\right)=\varepsilon g_{\alpha}(x, t), & g_{3}^{\varepsilon}\left(x^{\varepsilon}, t\right)=\varepsilon^{2} g_{3}(x, t), & x \in \Gamma_{ \pm}, \\
\rho_{e}^{\varepsilon}\left(x^{\varepsilon}, t\right)=\rho_{e}(x, t), & r^{\varepsilon}\left(x^{\varepsilon}, t\right)=r(x, t), & x \in \Omega \\
d^{\varepsilon}\left(x^{\varepsilon}, t\right)=d(x, t), & b^{\varepsilon}\left(x^{\varepsilon}, t\right)=b(x, t), & \varrho^{\varepsilon}\left(x^{\varepsilon}, t\right)=\varrho(x, t), \quad x \in \Gamma_{1}, \\
d^{\varepsilon}\left(x^{\varepsilon}, t\right)=\varepsilon d(x, t), & b^{\varepsilon}\left(x^{\varepsilon}, t\right)=\varepsilon b(x, t), & \varrho^{\varepsilon}\left(x^{\varepsilon}, t\right)=\varepsilon \varrho(x, t), x \in \Gamma_{ \pm} .
\end{array}
$$


We assume the following scalings for the mass densities $\rho_{i}^{\varepsilon}$, as in [7] (see also [1]):

$$
\rho_{\alpha}^{\varepsilon}\left(x^{\varepsilon}\right)=\rho(x), \quad \rho_{3}^{\varepsilon}\left(x^{\varepsilon}\right)=\varepsilon^{2} \rho(x), \quad x \in \Omega .
$$

Remark 2.1. The in-plane and transversal components of the mass density tensor are scaled differently. These assumptions aim at obtaining a scaled evolution problem that couples the three components of the displacement field. In particular [1], the $\varepsilon^{2}$ dependence of $\rho_{3}^{\varepsilon}$ allows, as an example, for an upward shift in the purely elastic transversal vibration frequencies of the plate as the scaling parameter goes to zero. Thus, the limit model is sensitive to inertia effects along the transversal direction, as it will be shown in the presentation of the flexural problems.

We distinguish the four cases of study for what concerns the scalings of the unknowns and test functions. In particular, since the mechanical and thermal loads and boundary conditions remain unvaried in any case, the scalings of the unknown displacements $u_{i}^{\varepsilon}$ and temperature $\theta^{\varepsilon}$ and their associated test functions shall always be

$$
\begin{array}{ll}
u_{\alpha}^{\varepsilon}\left(x^{\varepsilon}, t\right)=u_{\alpha}(\varepsilon)(x, t) & \text { for all } x^{\varepsilon}=\pi^{\varepsilon} x \in \bar{\Omega}^{\varepsilon}, t \in(0, T), \\
u_{3}^{\varepsilon}\left(x^{\varepsilon}, t\right)=\varepsilon^{-1} u_{3}(\varepsilon)(x, t) & \text { for all } x^{\varepsilon}=\pi^{\varepsilon} x \in \bar{\Omega}^{\varepsilon}, t \in(0, T), \\
\theta^{\varepsilon}\left(x^{\varepsilon}, t\right)=\theta(\varepsilon)(x, t) & \text { for all } x^{\varepsilon}=\pi^{\varepsilon} x \in \bar{\Omega}^{\varepsilon}, t \in(0, T),
\end{array}
$$

hence the associated scaled strain tensor field $\boldsymbol{\kappa}(\varepsilon)=\left(\kappa_{i j}(\varepsilon)\right)$, with $\kappa_{i j}(\varepsilon) \in L^{2}(\Omega)$ and scaled temperature gradient $\gamma(\varepsilon)=\left(\gamma_{i}(\varepsilon)\right)$, with $\gamma_{i}(\varepsilon) \in L^{2}(\Omega)$ are always given by

$$
\begin{aligned}
& \kappa_{\alpha \beta}(\varepsilon):=e_{\alpha \beta}(\mathbf{u}(\varepsilon)), \kappa_{\alpha 3}(\varepsilon):=\frac{1}{\varepsilon} e_{\alpha 3}(\mathbf{u}(\varepsilon)), \kappa_{33}(\varepsilon):=\frac{1}{\varepsilon^{2}} e_{33}(\mathbf{u}(\varepsilon)), \\
& \gamma_{\alpha}(\varepsilon):=\partial_{\alpha} \theta(\varepsilon), \quad \gamma_{3}(\varepsilon):=\frac{1}{\varepsilon} \partial_{3} \theta(\varepsilon) .
\end{aligned}
$$

Due to the different electromagnetic source terms and boundary conditions (see, e.g., [14]), the scalings related to the electric and magnetic potentials $\varphi^{\varepsilon}$ and $\zeta^{\varepsilon}$ shall vary throughout the asymptotic procedure; of course, the same holds for the scalings of the corresponding test functions. The scaled gradients of the electric and magnetic potentials will be denoted, respectively, by $\boldsymbol{\tau}(\varepsilon)=\left(\tau_{i}(\varepsilon)\right)$, with $\tau_{i}(\varepsilon) \in L^{2}(\Omega)$, and $\chi(\varepsilon)=\left(\chi_{i}(\varepsilon)\right)$, with $\chi_{i}(\varepsilon) \in L^{2}(\Omega)$.

In general, with an arbitrary state $\mathcal{V}=(\mathbf{v}, \psi, \xi, \eta)$, we associate, respectively, the tensor field $\boldsymbol{\kappa}(\varepsilon ; \mathbf{v})=\left(\kappa_{i j}(\varepsilon ; \mathbf{v})\right)$ and vector fields $\boldsymbol{\tau}(\varepsilon ; \psi)=\left(\tau_{i}(\varepsilon ; \psi)\right), \chi(\varepsilon ; \xi)=\left(\chi_{i}(\varepsilon ; \xi)\right)$ and $\gamma(\varepsilon ; \eta)=\left(\gamma_{i}(\varepsilon ; \eta)\right)$.

1) The sensor-actuator model. With the unknown state $\mathcal{U}^{\varepsilon} \in \mathbf{H}^{1}\left(\Omega^{\varepsilon}, \Gamma_{0}^{\varepsilon}\right) \times H^{1}\left(\Omega^{\varepsilon}, \Gamma_{0}^{\varepsilon}\right) \times$ $H^{1}\left(\Omega^{\varepsilon}, \Gamma_{ \pm}^{\varepsilon}\right) \times H^{1}\left(\Omega^{\varepsilon}, \Gamma_{0}^{\varepsilon}\right)$, we associate the scaled state $\mathcal{U}(\varepsilon):=(\mathbf{u}(\varepsilon), \varphi(\varepsilon), \bar{\zeta}(\varepsilon), \theta(\varepsilon)) \in$ $\mathbf{H}^{1}\left(\Omega, \Gamma_{0}\right) \times H^{1}\left(\Omega, \Gamma_{0}\right) \times H^{1}\left(\Omega, \Gamma_{ \pm}\right) \times H^{1}\left(\Omega, \Gamma_{0}\right)$, where

$$
\begin{aligned}
& \varphi^{\varepsilon}\left(x^{\varepsilon}, t\right)=\varphi(\varepsilon)(x, t) \text { for all } x^{\varepsilon}=\pi^{\varepsilon} x \in \bar{\Omega}^{\varepsilon}, t \in(0, T), \\
& \bar{\zeta}^{\varepsilon}\left(x^{\varepsilon}, t\right)=\varepsilon \bar{\zeta}(\varepsilon)(x, t) \text { for all } x^{\varepsilon}=\pi^{\varepsilon} x \in \bar{\Omega}^{\varepsilon}, t \in(0, T) .
\end{aligned}
$$

Moreover, with these scalings we associate, respectively,

$$
\begin{aligned}
& \tau_{\alpha}(\varepsilon):=\partial_{\alpha} \varphi(\varepsilon), \quad \tau_{3}(\varepsilon):=\frac{1}{\varepsilon} \partial_{3} \varphi(\varepsilon), \\
& \chi_{\alpha}(\varepsilon):=\varepsilon \partial_{\alpha} \zeta(\varepsilon), \chi_{3}(\varepsilon):=\partial_{3} \zeta(\varepsilon),
\end{aligned}
$$

We let $\bar{\chi}(\varepsilon):=\chi(\varepsilon ; \bar{\zeta}(\varepsilon))$ and $\widehat{\chi}:=\nabla \widehat{\zeta}$. 
We can now reformulate the problem on the fixed domain $\Omega$. For every $\varepsilon>0$ the scaled state $\mathcal{U}(\varepsilon):=(\mathbf{u}(\varepsilon), \varphi(\varepsilon), \bar{\zeta}(\varepsilon), \theta(\varepsilon))$ is the unique solution to the scaled problem:

$$
\left\{\begin{array}{l}
\text { Find } \mathcal{U}(\varepsilon) \in \mathbf{H}^{1}\left(\Omega, \Gamma_{0}\right) \times H^{1}\left(\Omega, \Gamma_{0}\right) \times H^{1}\left(\Omega, \Gamma_{ \pm}\right) \times H^{1}\left(\Omega, \Gamma_{0}\right), \\
\text { such that, for all } \mathcal{V} \in \mathbf{H}^{1}\left(\Omega, \Gamma_{0}\right) \times H^{1}\left(\Omega, \Gamma_{0}\right) \times H^{1}\left(\Omega, \Gamma_{ \pm}\right) \times H^{1}\left(\Omega, \Gamma_{0}\right), \\
A(\varepsilon)(\mathcal{U}(\varepsilon)(t), \mathcal{V})=L_{1}(\varepsilon)(\mathcal{V}), t \in(0, T)
\end{array}\right.
$$

with initial conditions $\left(\mathbf{u}_{0}, \mathbf{u}_{1}, \theta_{0}\right)$, where

$$
\begin{aligned}
& A(\varepsilon)(\mathcal{U}(\varepsilon)(t), \mathcal{V}):=(\rho \ddot{\mathbf{u}}(\varepsilon), \mathbf{v})+c(\varepsilon)(\eta, \dot{\mathbf{u}}(\varepsilon))+c_{v}(\dot{\theta}(\varepsilon), \eta)-d(\varepsilon)(\eta, \dot{\varphi}(\varepsilon))+ \\
&- e(\varepsilon)(\eta, \dot{\zeta}(\varepsilon))+a_{u}(\varepsilon)(\mathbf{u}(\varepsilon), \mathbf{v})+b(\varepsilon)(\varphi(\varepsilon), \mathbf{v})-b(\varepsilon)(\psi, \mathbf{u}(\varepsilon))+ \\
&+f(\varepsilon)(\zeta(\varepsilon), \mathbf{v})-f(\varepsilon)(\xi, \mathbf{u}(\varepsilon))-c(\varepsilon)(\theta(\varepsilon), \mathbf{v})+a_{\varphi}(\varepsilon)(\varphi(\varepsilon), \psi)+ \\
&+a_{\zeta}(\varepsilon)(\zeta(\varepsilon), \xi)+g(\varepsilon)(\zeta(\varepsilon), \psi)+g(\varepsilon)(\varphi(\varepsilon), \xi)-d(\varepsilon)(\theta(\varepsilon), \psi)+ \\
&-e(\varepsilon)(\theta(\varepsilon), \xi)+a_{\theta}(\varepsilon)(\theta(\varepsilon), \eta), \\
& L_{1}(\varepsilon)(\mathcal{V}):=(\mathbf{f}, \mathbf{v})+(\mathbf{g}, \mathbf{v})_{\mathbf{L}^{2}(\widehat{\Gamma})}+(r, \eta)-(\varrho, \eta)_{L^{2}(\widehat{\Gamma})}+\left(\rho_{e}, \psi\right)-(d, \psi)_{L^{2}(\widehat{\Gamma})}+ \\
&-a_{\zeta}(\varepsilon)(\widehat{\zeta}, \xi)-f(\varepsilon)(\widehat{\zeta}, \mathbf{v})+e(\varepsilon)(\eta, \dot{\widehat{\zeta}})-g(\varepsilon)(\widehat{\zeta}, \psi) .
\end{aligned}
$$

The new bilinear forms are defined as follows:

$$
\begin{array}{rlrl}
a_{u}(\varepsilon)(\mathbf{u}(\varepsilon), \mathbf{v}) & :=\int_{\Omega} C_{i j k \ell} \kappa_{k \ell}(\varepsilon) \kappa_{i j}(\varepsilon ; \mathbf{v}) d x, & a_{\varphi}(\varepsilon)(\varphi(\varepsilon), \psi):=\int_{\Omega} X_{i j} \tau_{j}(\varepsilon) \tau_{i}(\varepsilon ; \psi) d x \\
a_{\zeta}(\varepsilon)(\zeta(\varepsilon), \xi):=\int_{\Omega} M_{i j} \chi_{j}(\varepsilon) \chi_{i}(\varepsilon ; \xi) d x, & & a_{\theta}(\varepsilon)(\theta(\varepsilon), \eta):=\frac{1}{T_{0}} \int_{\Omega} K_{i j} \gamma_{j}(\varepsilon) \gamma_{i}(\varepsilon ; \eta) d x \\
b(\varepsilon)(\psi, \mathbf{u}(\varepsilon)):=\int_{\Omega} P_{k i j} \tau_{k}(\varepsilon ; \psi) \kappa_{i j}(\varepsilon) d x, & c(\varepsilon)(\eta, \mathbf{u}(\varepsilon)):=\int_{\Omega} \eta \beta_{i j} \kappa_{i j}(\varepsilon) d x, \\
d(\varepsilon)(\eta, \varphi(\varepsilon)):=\int_{\Omega} \eta p_{k} \tau_{k}(\varepsilon) d x, & e(\varepsilon)(\eta, \zeta(\varepsilon)):=\int_{\Omega} \eta m_{k} \chi_{k}(\varepsilon) d x \\
f(\varepsilon)(\xi, \mathbf{u}(\varepsilon)):=\int_{\Omega} R_{k i j} \chi_{k}(\varepsilon ; \xi) \kappa_{i j}(\varepsilon) d x, & g(\varepsilon)(\zeta(\varepsilon), \psi):=\int_{\Omega} \alpha_{i j} \chi_{j}(\varepsilon) \tau_{i}(\varepsilon ; \psi) d x .
\end{array}
$$

The scaled energy $\mathcal{E}(\varepsilon)(t)$ associated with a weak solution $\mathcal{U}(\varepsilon)$ is:

$$
\begin{aligned}
\mathcal{E}(\varepsilon)(t):=\frac{1}{2}\left\{(\rho \dot{\mathbf{u}}(\varepsilon), \dot{\mathbf{u}}(\varepsilon))+a_{u}(\varepsilon)(\mathbf{u}(\varepsilon), \mathbf{u}(\varepsilon))+a_{\varphi}(\varepsilon)(\varphi(\varepsilon), \varphi(\varepsilon))+a_{\zeta}(\varepsilon)(\zeta(\varepsilon), \zeta(\varepsilon))+\right. \\
\left.+\left(c_{v} \theta(\varepsilon), \theta(\varepsilon)\right)-2 d(\varepsilon)(\theta(\varepsilon), \varphi(\varepsilon))-2 e(\varepsilon)(\theta(\varepsilon), \zeta(\varepsilon))+2 g(\varepsilon)(\zeta(\varepsilon), \varphi(\varepsilon))\right\} .
\end{aligned}
$$

Following [2], the scaled energy associated with a weak solution $\mathcal{U}(\varepsilon)$ of $(2.5)$, satisfies:

$$
\dot{\mathcal{E}}(\varepsilon)(t)+a_{\theta}(\varepsilon)(\theta(\varepsilon), \theta(\varepsilon))=L_{\mathcal{E}}^{1}(\varepsilon)(t),
$$

with

$$
\begin{aligned}
L_{\mathcal{E}}^{1}(\varepsilon)(t):= & (\mathbf{f}, \dot{\mathbf{u}}(\varepsilon))+(\mathbf{g}, \dot{\mathbf{u}}(\varepsilon))_{\mathbf{L}^{2}(\widehat{\Gamma})}+\left(\dot{\rho}_{e}, \varphi(\varepsilon)\right)-(\dot{d}, \varphi(\varepsilon))_{L^{2}(\widehat{\Gamma})}+ \\
& +(r, \theta(\varepsilon))-(\varrho, \theta(\varepsilon))_{L^{2}(\widehat{\Gamma})}-a_{\zeta}(\varepsilon)(\dot{\widehat{\zeta}}, \bar{\zeta}(\varepsilon))-f(\varepsilon)(\widehat{\zeta}, \dot{\mathbf{u}}(\varepsilon))+ \\
& +e(\varepsilon)(\theta(\varepsilon),, \dot{\widehat{\zeta}})-g(\varepsilon)(\dot{\widehat{\zeta}}, \varphi(\varepsilon)) .
\end{aligned}
$$

2) The actuator-sensor model. We assume the following scalings for $\varphi^{\varepsilon}$ and $\zeta^{\varepsilon}$ :

$$
\begin{aligned}
& \bar{\varphi}^{\varepsilon}\left(x^{\varepsilon}, t\right)=\varepsilon \bar{\varphi}(\varepsilon)(x, t) \text { for all } x^{\varepsilon}=\pi^{\varepsilon} x \in \bar{\Omega}^{\varepsilon}, t \in(0, T), \\
& \zeta^{\varepsilon}\left(x^{\varepsilon}, t\right)=\zeta(\varepsilon)(x, t) \text { for all } x^{\varepsilon}=\pi^{\varepsilon} x \in \bar{\Omega}^{\varepsilon}, t \in(0, T) .
\end{aligned}
$$


Moreover, the components of the scaled vector fields $\boldsymbol{\tau}(\varepsilon)=\left(\tau_{i}(\varepsilon)\right)$ and $\chi(\varepsilon)=\left(\chi_{i}(\varepsilon)\right)$ are now defined by

$$
\begin{aligned}
& \tau_{\alpha}(\varepsilon):=\varepsilon \partial_{\alpha} \varphi(\varepsilon), \tau_{3}(\varepsilon):=\partial_{3} \varphi(\varepsilon), \\
& \chi_{\alpha}(\varepsilon):=\partial_{\alpha} \zeta(\varepsilon), \quad \chi_{3}(\varepsilon):=\frac{1}{\varepsilon} \partial_{3} \zeta(\varepsilon) .
\end{aligned}
$$

We let $\overline{\boldsymbol{\tau}}(\varepsilon):=\boldsymbol{\tau}(\varepsilon ; \bar{\varphi}(\varepsilon))$ and $\widehat{\boldsymbol{\tau}}:=\nabla \widehat{\varphi}$. The scaled state $\mathcal{U}(\varepsilon):=(\mathbf{u}(\varepsilon), \bar{\varphi}(\varepsilon), \zeta(\varepsilon), \theta(\varepsilon))$ is the unique solution to the scaled problem:

$$
\left\{\begin{array}{l}
\text { Find } \mathcal{U}(\varepsilon) \in \mathbf{H}^{1}\left(\Omega, \Gamma_{0}\right) \times H^{1}\left(\Omega, \Gamma_{ \pm}\right) \times H^{1}\left(\Omega, \Gamma_{0}\right) \times H^{1}\left(\Omega, \Gamma_{0}\right), \text { such that } \\
\text { for all } \mathcal{V} \in \mathbf{H}^{1}\left(\Omega, \Gamma_{0}\right) \times H^{1}\left(\Omega, \Gamma_{ \pm}\right) \times H^{1}\left(\Omega, \Gamma_{0}\right) \times H^{1}\left(\Omega, \Gamma_{0}\right), \\
A(\varepsilon) \mathcal{U}(\varepsilon)(t), \mathcal{V})=L_{2}(\varepsilon)(\mathcal{V}), t \in(0, T)
\end{array}\right.
$$

with initial conditions $\left(\mathbf{u}_{0}, \mathbf{u}_{1}, \theta_{0}\right)$, where

$$
\begin{aligned}
L_{2}(\varepsilon)(\mathcal{V}) & :=(\mathbf{f}, \mathbf{v})+(\mathbf{g}, \mathbf{v})_{\mathbf{L}^{2}(\widehat{\Gamma})}+(r, \eta)-(\varrho, \eta)_{L^{2}(\widehat{\Gamma})}+\left(\rho_{e}, \psi\right)-(b, \xi)_{L^{2}(\widehat{\Gamma})}+ \\
& -a_{\varphi}(\varepsilon)(\widehat{\varphi}, \psi)-b(\varepsilon)(\widehat{\varphi}, \mathbf{v})+d(\varepsilon)(\eta, \dot{\widehat{\varphi}})-g(\varepsilon)(\xi, \widehat{\varphi}) .
\end{aligned}
$$

3) The actuator model. We assume the following scalings for $\varphi^{\varepsilon}$ and $\zeta^{\varepsilon}$ :

$$
\begin{aligned}
& \bar{\varphi}^{\varepsilon}\left(x^{\varepsilon}, t\right)=\varepsilon \bar{\varphi}(\varepsilon)(x, t) \text { for all } x^{\varepsilon}=\pi^{\varepsilon} x \in \bar{\Omega}^{\varepsilon}, t \in(0, T), \\
& \bar{\zeta}^{\varepsilon}\left(x^{\varepsilon}, t\right)=\varepsilon \bar{\zeta}(\varepsilon)(x, t) \text { for all } x^{\varepsilon}=\pi^{\varepsilon} x \in \bar{\Omega}^{\varepsilon}, t \in(0, T) .
\end{aligned}
$$

Moreover, the components of the scaled vector fields $\boldsymbol{\tau}(\varepsilon)=\left(\tau_{i}(\varepsilon)\right)$ and $\chi(\varepsilon)=\left(\chi_{i}(\varepsilon)\right)$ are now defined by

$$
\begin{aligned}
& \tau_{\alpha}(\varepsilon):=\varepsilon \partial_{\alpha} \varphi(\varepsilon), \tau_{3}(\varepsilon):=\partial_{3} \varphi(\varepsilon) \\
& \chi_{\alpha}(\varepsilon):=\varepsilon \partial_{\alpha} \zeta(\varepsilon), \chi_{3}(\varepsilon):=\partial_{3} \zeta(\varepsilon) .
\end{aligned}
$$

The scaled state $\mathcal{U}(\varepsilon):=(\mathbf{u}(\varepsilon), \bar{\varphi}(\varepsilon), \bar{\zeta}(\varepsilon), \theta(\varepsilon))$ is the unique solution to the scaled problem:

$$
\left\{\begin{array}{l}
\text { Find } \mathcal{U}(\varepsilon) \in \mathbf{H}^{1}\left(\Omega, \Gamma_{0}\right) \times H^{1}\left(\Omega, \Gamma_{ \pm}\right) \times H^{1}\left(\Omega, \Gamma_{ \pm}\right) \times H^{1}\left(\Omega, \Gamma_{0}\right), \text { such that } \\
\text { for all } \mathcal{V} \in \mathbf{H}^{1}\left(\Omega, \Gamma_{0}\right) \times H^{1}\left(\Omega, \Gamma_{ \pm}\right) \times H^{1}\left(\Omega, \Gamma_{ \pm}\right) \times H^{1}\left(\Omega, \Gamma_{0}\right), \\
A(\varepsilon)(\mathcal{U}(\varepsilon)(t), \mathcal{V})=L_{3}(\varepsilon)(\mathcal{V}), t \in(0, T)
\end{array}\right.
$$

with initial conditions $\left(\mathbf{u}_{0}, \mathbf{u}_{1}, \theta_{0}\right)$, where

$$
\begin{aligned}
L_{3}(\varepsilon)(\mathcal{V}): & =(\mathbf{f}, \mathbf{v})+(\mathbf{g}, \mathbf{v})_{\mathbf{L}^{2}(\widehat{\Gamma})}+(r, \eta)-(\varrho, \eta)_{L^{2}(\widehat{\Gamma})}+\left(\rho_{e}, \psi\right)+ \\
& -a_{\zeta}(\varepsilon)(\widehat{\zeta}, \psi)-f(\varepsilon)(\widehat{\zeta}, \mathbf{v})+e(\varepsilon)(\eta, \dot{\widehat{\zeta}})-g(\varepsilon)(\widehat{\zeta}, \psi)+ \\
& -a_{\varphi}(\varepsilon)(\widehat{\varphi}, \psi)-b(\varepsilon)(\widehat{\varphi}, \mathbf{v})+d(\varepsilon)(\eta, \dot{\widehat{\varphi}})-g(\varepsilon)(\xi, \widehat{\varphi})
\end{aligned}
$$

4) The sensor model. We assume the following scalings for $\varphi^{\varepsilon}$ and $\zeta^{\varepsilon}$ :

$$
\begin{aligned}
& \varphi^{\varepsilon}\left(x^{\varepsilon}, t\right)=\varphi(\varepsilon)(x, t) \text { for all } x^{\varepsilon}=\pi^{\varepsilon} x \in \bar{\Omega}^{\varepsilon}, t \in(0, T), \\
& \zeta^{\varepsilon}\left(x^{\varepsilon}, t\right)=\zeta(\varepsilon)(x, t) \text { for all } x^{\varepsilon}=\pi^{\varepsilon} x \in \bar{\Omega}^{\varepsilon}, t \in(0, T) .
\end{aligned}
$$

Moreover, the components of the scaled vector fields $\boldsymbol{\tau}(\varepsilon)=\left(\tau_{i}(\varepsilon)\right)$ and $\boldsymbol{\chi}(\varepsilon)=\left(\chi_{i}(\varepsilon)\right)$ are now defined by

$$
\begin{aligned}
& \tau_{\alpha}(\varepsilon):=\partial_{\alpha} \varphi(\varepsilon), \tau_{3}(\varepsilon):=\frac{1}{\varepsilon} \partial_{3} \varphi(\varepsilon) \\
& \chi_{\alpha}(\varepsilon):=\partial_{\alpha} \zeta(\varepsilon), \chi_{3}(\varepsilon):=\frac{1}{\varepsilon} \partial_{3} \zeta(\varepsilon)
\end{aligned}
$$


The scaled state $\mathcal{U}(\varepsilon):=(\mathbf{u}(\varepsilon), \varphi(\varepsilon), \zeta(\varepsilon), \theta(\varepsilon))$ is the unique solution to the scaled problem:

$$
\left\{\begin{array}{l}
\text { Find } \mathcal{U}(\varepsilon) \in \mathbf{H}^{1}\left(\Omega, \Gamma_{0}\right) \times H^{1}\left(\Omega, \Gamma_{0}\right) \times H^{1}\left(\Omega, \Gamma_{0}\right) \times H^{1}\left(\Omega, \Gamma_{0}\right), \text { such that } \\
\text { for all } \mathcal{V}=(\mathbf{v}, \psi, \xi, \eta) \in \mathbf{H}^{1}\left(\Omega, \Gamma_{0}\right) \times H^{1}\left(\Omega, \Gamma_{0}\right) \times H^{1}\left(\Omega, \Gamma_{0}\right) \times H^{1}\left(\Omega, \Gamma_{0}\right), \\
A(\varepsilon)(\mathcal{U}(\varepsilon)(t), \mathcal{V})=L_{4}(\varepsilon)(\mathcal{V}), t \in(0, T),
\end{array}\right.
$$

with initial conditions $\left(\mathbf{u}_{0}, \mathbf{u}_{1}, \theta_{0}\right)$, where

$$
L_{4}(\varepsilon)(\mathcal{V}):=(\mathbf{f}, \mathbf{v})+(\mathbf{g}, \mathbf{v})_{\mathbf{L}^{2}(\widehat{\Gamma})}+(r, \eta)-(\varrho, \eta)_{L^{2}(\widehat{\Gamma})}+\left(\rho_{e}, \psi\right)-(b, \xi)_{L^{2}(\widehat{\Gamma})}-(d, \psi)_{L^{2}(\widehat{\Gamma})} .
$$

\section{Convergence Results}

Preliminarily, we introduce the functional spaces

$$
\begin{aligned}
& \mathbb{X}(\Omega):=\left\{\xi \in L^{2}(\Omega), \partial_{3} \xi \in L^{2}(\Omega)\right\} \equiv H^{1}\left(-h, h ; L^{2}(\omega)\right), \\
& \mathbb{X}_{0}(\Omega):=\left\{\xi \in L^{2}(\Omega), \partial_{3} \xi \in L^{2}(\Omega), \xi=0 \text { on } \Gamma_{ \pm}\right\}
\end{aligned}
$$

usual in the asymptotic analysis of actuator piezoelectric plates (see, e.g., [11]). Also, let

$$
\mathbf{V}_{K L}(\Omega):=\left\{\mathbf{v} \in \mathbf{H}^{1}\left(\Omega, \Gamma_{0}\right) ; e_{i 3}(\mathbf{v})=0\right\}
$$

denote the space of Kirchhoff-Love displacements, and

$$
\begin{aligned}
& \mathbf{V}_{H}\left(\omega, \gamma_{0}\right):=\left\{\mathbf{v}_{H}=\left(v_{\alpha}\right) \in \mathbf{H}^{1}(\omega) ; \mathbf{v}_{H}=\mathbf{0} \text { on } \gamma_{0}\right\}, \\
& V_{3}\left(\omega, \gamma_{0}\right):=\left\{v_{3} \in H^{2}(\omega) ; v_{3}=0 \text { and } \partial_{\nu} v_{3}=0 \text { on } \gamma_{0}\right\},
\end{aligned}
$$

where $\boldsymbol{\nu}=\left(\nu_{\alpha}\right)$ is the outer unit normal vector to $\gamma$. We recall that $\boldsymbol{\tau}=\left(-\nu_{2}, \nu_{1}\right)$ represents the unit tangent vector to $\gamma$.

As we shall prove in the sequel, the limit displacement field is always a Kirchhoff-Love field; thus, for consistency reasons, we consider initial conditions such that (see [7])

$$
\left\{\begin{array}{l}
\mathbf{u}(\varepsilon)(0)=\mathbf{u}_{0} \in \mathbf{H}^{2}(\Omega) \cap \mathbf{V}_{K L}(\Omega), \\
\partial_{t} \mathbf{u}(\varepsilon)(0)=\mathbf{u}_{1} \in \mathbf{V}_{K L}(\Omega), \\
\theta(\varepsilon)(0)=\theta_{0} \in H^{2}(\Omega) \cap H^{1}\left(\Omega, \Gamma_{0}\right) .
\end{array}\right.
$$

\subsection{The Sensor-Actuator model}

Theorem 3.1. Under assumption (3.2), the sequence $\{\mathcal{U}(\varepsilon)\}_{\varepsilon>0}$ weakly converges to the limit $\tilde{\mathcal{U}}:=(\tilde{\mathbf{u}}, \tilde{\varphi}, \tilde{\zeta}, \tilde{\theta})$ in the space $L^{2}\left(0, T ; \mathbf{H}^{1}(\Omega)\right) \times L^{2}\left(0, T ; H^{1}(\Omega)\right) \times L^{2}(0, T ; \mathbb{X}(\Omega)) \times$ $L^{2}\left(0, T ; H^{1}(\Omega)\right)$.

Proof. For the sake of clarity, the proof is divided into four parts. The first three parts are devoted to showing that the sequence associated with the scaled energy $\{\mathcal{E}(\varepsilon)(t)\}_{\varepsilon>0}$ is uniformly bounded; the proof of the weak convergence result is then accomplished in the fourth part.

(i) Bounds on $L_{\mathcal{E}}^{1}(\varepsilon)$. First we rewrite $(\mathbf{g}, \dot{\mathbf{u}}(\varepsilon))_{\mathbf{L}^{2}(\widehat{\Gamma})}=\partial_{t}(\mathbf{g}, \mathbf{u}(\varepsilon))_{\mathbf{L}^{2}(\widehat{\Gamma})}-(\dot{\mathbf{g}}, \mathbf{u}(\varepsilon))_{\mathbf{L}^{2}(\widehat{\Gamma})}$ and $f(\varepsilon)(\widehat{\zeta}, \dot{\mathbf{u}}(\varepsilon))=\partial_{t}[f(\varepsilon)(\widehat{\zeta}, \mathbf{u}(\varepsilon))]-f(\varepsilon)(\dot{\widehat{\zeta}}, \mathbf{u}(\varepsilon))$. By using, in expression (2.8), CauchySchwarz, Poincaré's, Korn's and Young's inequalities, along with the continuity of the trace operator, we obtain the existence of positive constants $C_{1}$ and $\delta_{0}$ such that

$$
\begin{aligned}
L_{\mathcal{E}}^{1}(\varepsilon)(\mathcal{U}(\varepsilon)) \leq & C_{0}(t)+\frac{C_{1}}{2}\left\{|\dot{\mathbf{u}}(\varepsilon)|_{0, \Omega}^{2}+|\boldsymbol{\kappa}(\varepsilon)|_{0, \Omega}^{2}+|\boldsymbol{\tau}(\varepsilon)|_{0, \Omega}^{2}+|\theta(\varepsilon)|_{0, \Omega}^{2}+|\overline{\boldsymbol{\chi}}(\varepsilon)|_{0, \Omega}^{2}+\right. \\
& \left.+\delta_{0}|\boldsymbol{\gamma}(\varepsilon)|^{2}\right\}+\partial_{t}(\mathbf{g}, \mathbf{u}(\varepsilon))_{\mathbf{L}^{2}(\widehat{\Gamma})}-\partial_{t}[f(\varepsilon)(\widehat{\zeta}, \mathbf{u}(\varepsilon))]
\end{aligned}
$$


where $2 C_{0}(t):=|\mathbf{f}|_{0, \Omega}^{2}+|\dot{\mathbf{g}}|_{0, \widehat{\Gamma}}^{2}+\left.|\dot{\rho}|_{e}\right|_{0, \Omega} ^{2}+|\dot{d}|_{0, \widehat{\Gamma}}^{2}+|r|_{0, \Omega}^{2}+\frac{1}{\delta_{0}}|\varrho|_{0, \hat{\Gamma}}^{2}+|\dot{\widehat{\chi}}|_{0, \Omega}^{2}$ depends on the domain and the data (not on $\varepsilon$ ). Denoting by $K>0$ the coercivity constant of $a_{\theta}(\cdot, \cdot)$, with a view toward applying $(2.7)$, we choose $\delta_{0}$ such that $\widetilde{K}:=K-\frac{C_{1} \delta_{0}}{2}>0$. By the definition of the scaled energy and the positive definiteness hypothesis of $\mathbb{M}^{c}$ (subsection 1.1), there exists a positive constant $C_{2}$ such that

$$
|\dot{\mathbf{u}}(\varepsilon)|_{0, \Omega}^{2}+|\boldsymbol{\kappa}(\varepsilon)|_{0, \Omega}^{2}+|\boldsymbol{\tau}(\varepsilon)|_{0, \Omega}^{2}+|\theta(\varepsilon)|_{0, \Omega}^{2}+|\bar{\chi}(\varepsilon)|_{0, \Omega}^{2} \leq C_{2} \mathcal{E}(\varepsilon),
$$

hence there exists $C_{3}>0$ such that

$$
L_{\mathcal{E}}^{1}(\varepsilon) \leq C_{0}(t)+C_{3} \mathcal{E}(\varepsilon)+\partial_{t}(\mathbf{g}, \mathbf{u}(\varepsilon))_{\mathbf{L}^{2}(\widehat{\Gamma})}-\partial_{t}[f(\varepsilon)(\widehat{\zeta}, \mathbf{u}(\varepsilon))] .
$$

(ii) The sequence $\{\mathcal{E}(\varepsilon)(0)\}_{\varepsilon>0}$ is uniformly bounded. We have:

$$
\begin{aligned}
2 \mathcal{E}(\varepsilon)(0)=( & \left.\rho \mathbf{u}_{1}, \mathbf{u}_{1}\right)+a_{u}(\varepsilon)\left(\mathbf{u}_{0}, \mathbf{u}_{0}\right)+a_{\varphi}(\varepsilon)\left(\varphi_{0}(\varepsilon), \varphi_{0}(\varepsilon)\right)+a_{\zeta}(\varepsilon)\left(\bar{\zeta}_{0}(\varepsilon), \bar{\zeta}_{0}(\varepsilon)\right)+ \\
& +\left(c_{v} \theta_{0}, \theta_{0}\right)-2 d(\varepsilon)\left(\theta_{0}, \varphi_{0}(\varepsilon)\right)-2 e(\varepsilon)\left(\theta_{0}, \bar{\zeta}_{0}(\varepsilon)\right)+2 g(\varepsilon)\left(\bar{\zeta}_{0}(\varepsilon), \varphi_{0}(\varepsilon)\right),
\end{aligned}
$$

where $\left(\varphi_{0}(\varepsilon), \bar{\zeta}_{0}(\varepsilon)\right)$ is the solution to the following variational problem

$$
\left\{\begin{array}{l}
\text { Find }\left(\left(\varphi_{0}(\varepsilon), \bar{\zeta}_{0}(\varepsilon)\right) \in H^{1}\left(\Omega, \Gamma_{0}\right) \times H^{1}\left(\Omega, \Gamma_{ \pm}\right)\right. \\
\text {such that for all }(\psi, \xi) \in H^{1}\left(\Omega, \Gamma_{0}\right) \times H^{1}\left(\Omega, \Gamma_{ \pm}\right) \\
\begin{array}{rl}
a_{\varphi}(\varepsilon)\left(\varphi_{0}(\varepsilon), \psi\right)+a_{\zeta}(\varepsilon)\left(\bar{\zeta}_{0}(\varepsilon), \xi\right)+g(\varepsilon)\left(\bar{\zeta}_{0}(\varepsilon), \psi\right)+g(\varepsilon)\left(\varphi_{0}(\varepsilon), \xi\right)= \\
=\left(\rho_{e}(0), \psi\right)-(d(0), \psi) L_{2}(\widehat{\Gamma})-a_{\zeta}(\varepsilon)\left(\widehat{\zeta}_{0}, \xi\right)+f(\varepsilon)\left(\xi, \mathbf{u}_{0}\right)-e(\varepsilon)\left(\theta_{0}, \xi\right)+ \\
\quad-g(\varepsilon)\left(\widehat{\zeta}_{0}, \psi\right)+d(\varepsilon)\left(\theta_{0}, \psi\right)+b(\varepsilon)\left(\psi, \mathbf{u}_{0}\right)
\end{array}
\end{array}\right.
$$

By virtue of the definite positiveness hypothesis of $\mathbb{M}^{c}$ (with constant $c_{1}$ ), CauchySchwarz, Poincaré's and Korn's inequalities (with constant $c_{2}$ ) and Young's inequality (with constant $\delta_{1}$ ), one has that

$$
\begin{aligned}
& c_{1}\left\{\left|\boldsymbol{\tau}_{0}(\varepsilon)\right|_{0, \Omega}^{2}+\left|\overline{\boldsymbol{\chi}}_{0}(\varepsilon)\right|_{0, \Omega}^{2}\right\} \leq \\
& \leq a_{\varphi}(\varepsilon)\left(\varphi_{0}(\varepsilon), \varphi_{0}(\varepsilon)\right)+a_{\zeta}(\varepsilon)\left(\bar{\zeta}_{0}(\varepsilon), \bar{\zeta}_{0}(\varepsilon)\right)+2 g(\varepsilon)\left(\bar{\zeta}_{0}(\varepsilon), \varphi_{0}(\varepsilon)\right) \leq \\
& \leq c_{2}\left\{\left|\boldsymbol{\tau}_{0}(\varepsilon)\right|_{0, \Omega}\left(\left|\rho_{e}(0)\right|_{0, \Omega}+|d(0)|_{0, \hat{\Gamma}}+\left|\nabla \mathbf{u}_{0}\right|_{0, \Omega}+\left|\theta_{0}\right|_{0, \Omega}+\left|\nabla \widehat{\zeta}_{0}\right|_{0, \Omega}\right)+\right. \\
& \left.\quad+\left|\bar{\chi}_{0}(\varepsilon)\right|_{0, \Omega}\left(\left|\nabla \mathbf{u}_{0}\right|_{0, \Omega}+\left|\theta_{0}\right|_{0, \Omega}+\left|\nabla \widehat{\zeta}_{0}\right|_{0, \Omega}\right)\right\} \leq \\
& \leq \frac{c_{3} \delta_{1}}{2}\left\{\left|\boldsymbol{\tau}_{0}(\varepsilon)\right|_{0, \Omega}^{2}+\left|\bar{\chi}_{0}(\varepsilon)\right|_{0, \Omega}^{2}\right\}+ \\
& \quad+\frac{c_{3}}{2 \delta_{1}}\left\{\left|\rho_{e}(0)\right|_{0, \Omega}^{2}+|d(0)|_{0, \hat{\Gamma}}^{2}+\left|\nabla \mathbf{u}_{0}\right|_{0, \Omega}^{2}+\left|\theta_{0}\right|_{0, \Omega}^{2}+\left|\nabla \widehat{\zeta}_{0}\right|_{0, \Omega}^{2}\right\}
\end{aligned}
$$

Finally, on choosing $\delta_{1}$ such that $c_{1}-\frac{c_{3} \delta_{1}}{2}>0$, we get the uniform boundedness of $\left|\boldsymbol{\tau}_{0}(\varepsilon)\right|_{0, \Omega}$ and $\left|\bar{\chi}_{0}(\varepsilon)\right|_{0, \Omega}$. Now, we remark that $e_{i 3}\left(\mathbf{u}_{0}\right)=0$ by hypothesis (3.2), which implies

$$
a_{u}(\varepsilon)\left(\mathbf{u}_{0}, \mathbf{u}_{0}\right)=\int_{\Omega} C_{i j k \ell} \kappa_{k \ell}(\varepsilon)\left(\mathbf{u}_{0}\right) \kappa_{i j}(\varepsilon)\left(\mathbf{u}_{0}\right) d x=\int_{\Omega} C_{\alpha \beta \gamma \delta} \kappa_{\gamma \delta}(\varepsilon)\left(\mathbf{u}_{0}\right) \kappa_{\alpha \beta}(\varepsilon)\left(\mathbf{u}_{0}\right) d x
$$

so that, by the properties of $\mathbf{C}$, we have

$$
a_{u}(\varepsilon)\left(\mathbf{u}_{0}, \mathbf{u}_{0}\right) \leq c\left|\nabla \mathbf{u}_{0}\right|_{0, \Omega}^{2}
$$


for some constant $c>0$. Consequently, by virtue of the above bounds, there exists a constant $c_{4}>0$ such that $\mathcal{E}(\varepsilon)(0) \leq c_{4}$, i.e., the sequence $\{\mathcal{E}(\varepsilon)(0)\}_{\varepsilon>0}$ is uniformly bounded.

(iii) The sequence $\{\mathcal{E}(\varepsilon)(t)\}_{\varepsilon>0}$ is uniformly bounded. Upon integrating the energy evolution equation $(2.7)$ in $(0, t)$, using the results of steps (i) and (ii), the continuity of the trace operator and Korn's inequality, we infer that

$$
\begin{aligned}
& \mathcal{E}(\varepsilon)(t)+\widetilde{K} \int_{0}^{t}|\gamma(\varepsilon)(s)|_{0, \Omega}^{2} d s \leq \mathcal{E}(\varepsilon)(0)+\int_{0}^{t} L_{\mathcal{E}}^{1}(\varepsilon)(s) d s \leq \\
& \leq c_{4}+\int_{0}^{t}\left(C_{0}(s)+C_{3} \mathcal{E}(\varepsilon)(s)+\partial_{s}(\mathbf{g}(s), \mathbf{u}(\varepsilon)(s))_{\mathbf{L}^{2}(\widehat{\Gamma})}-\partial_{s}[f(\varepsilon)(\widehat{\zeta}(s), \mathbf{u}(\varepsilon)(s))]\right) d s \leq \\
& \leq C_{4}(t)+\frac{1}{2}\left(\frac{c_{5}}{\delta_{2}}|\widehat{\chi}(t)|_{0, \Omega}^{2}+\frac{c_{6}}{\delta_{3}}|\mathbf{g}(t)|_{0, \widehat{\Gamma}}^{2}\right)+\frac{1}{2}\left(c_{5} \delta_{2}+c_{6} \delta_{3}\right)|\boldsymbol{\kappa}(\varepsilon)(t)|_{0, \Omega}^{2}+C_{3} \int_{0}^{t} \mathcal{E}(\varepsilon)(s) d s .
\end{aligned}
$$

Since $|\boldsymbol{\kappa}(\varepsilon)(t)|_{0, \Omega}^{2}$ is bounded (up to a constant) by $\mathcal{E}(\varepsilon)(t)$, it is sufficient to select suitable values of $\delta_{2}$ and $\delta_{3}$ to get the following estimate:

$$
\mathcal{E}(\varepsilon)(t)+\widetilde{K} \int_{0}^{t}|\gamma(\varepsilon)(s)|_{0, \Omega}^{2} d s \leq C_{5}(t)+C_{3} \int_{0}^{t} \mathcal{E}(\varepsilon)(s) d s \leq C_{6}+C_{3} \int_{0}^{t} \mathcal{E}(\varepsilon)(s) d s,
$$

with $C_{6}:=\sup _{t \in(0, T)} C_{5}(t)$. Thanks to Gronwall's inequality, there exist two positive constants $m$ and $k$ such that

$$
\mathcal{E}(\varepsilon)(t) \leq m e^{k t} \text { and } \widetilde{K} \int_{0}^{t}|\gamma(\varepsilon)(s)|_{0, \Omega}^{2} d s \leq m e^{k t} \text { for all } t \in(0, T) .
$$

(iv) Weak convergences. We are now in a position to establish the weak convergence result. From the bound on the energy we infer that the sequences $\{\boldsymbol{\kappa}(\varepsilon)\}_{\varepsilon>0},\{\bar{\chi}(\varepsilon)\}_{\varepsilon>0}$, $\{\boldsymbol{\tau}(\varepsilon)\}_{\varepsilon>0}$ and $\{\boldsymbol{\gamma}(\varepsilon)\}_{\varepsilon>0}$ are uniformly bounded in $L^{2}\left(0, T ; \mathbf{L}^{2}(\Omega)\right)$, therefore we have the following weak convergences (up to a subsequence):

$$
\begin{array}{lll}
\kappa_{i j}(\varepsilon)-\tilde{\kappa}_{i j} & \text { in } & L^{2}\left(0, T ; L^{2}(\Omega)\right), \\
\bar{\chi}_{i}(\varepsilon) \rightarrow \tilde{\bar{\chi}}_{i} & \text { in } & L^{2}\left(0, T ; L^{2}(\Omega)\right), \\
\tau_{i}(\varepsilon) \rightarrow \tilde{\tau}_{i} \quad \text { in } & L^{2}\left(0, T ; L^{2}(\Omega)\right), \\
\gamma_{i}(\varepsilon) \rightarrow \tilde{\gamma}_{i} \quad \text { in } & L^{2}\left(0, T ; L^{2}(\Omega)\right) .
\end{array}
$$

Moreover, by means of Korn's and Poincaré's inequalities and from the definition of $\kappa_{i j}(\varepsilon)$, $\tau_{i}(\varepsilon)$ and $\gamma_{i}(\varepsilon)$, we infer that $\|\mathbf{u}(\varepsilon)\|_{1, \Omega},\|\varphi(\varepsilon)\|_{1, \Omega}$ and $\|\theta(\varepsilon)\|_{1, \Omega}$ are also bounded, so that

$$
\begin{array}{ll}
\mathbf{u}(\varepsilon) \rightarrow \tilde{\mathbf{u}} \text { in } & L^{2}\left(0, T ; \mathbf{H}^{1}(\Omega)\right), \\
\dot{\mathbf{u}}(\varepsilon) \rightarrow \tilde{\mathbf{u}} \text { in } & L^{2}\left(0, T ; \mathbf{L}^{2}(\Omega)\right), \\
\varphi(\varepsilon) \rightarrow \tilde{\varphi} \text { in } & L^{2}\left(0, T ; H^{1}(\Omega)\right), \\
\theta(\varepsilon) \rightarrow \tilde{\theta} \text { in } & L^{2}\left(0, T ; H^{1}(\Omega)\right)
\end{array}
$$

Upon writing

$$
\bar{\zeta}(\varepsilon)\left(\tilde{x}, x_{3}\right)=\int_{-h}^{x_{3}} \partial_{3} \bar{\zeta}(\varepsilon)\left(\tilde{x}, y_{3}\right) d y_{3},
$$

it follows that $|\bar{\zeta}(\varepsilon)|_{0, \Omega} \leq 2 h\left|\partial_{3} \bar{\zeta}(\varepsilon)\right|_{0, \Omega} \leq c e^{m T}$, by virtue of the boundedness of $\bar{\chi}_{i}(\varepsilon)$. This implies that both $\bar{\zeta}(\varepsilon)$ and $\zeta(\varepsilon)$ are bounded in $L^{2}(\Omega)$ and thus,

$$
\bar{\zeta}(\varepsilon) \rightarrow \tilde{\bar{\zeta}} \text { in } L^{2}\left(0, T ; \mathbb{X}_{0}(\Omega)\right), \quad \zeta(\varepsilon) \rightarrow \tilde{\zeta} \text { in } L^{2}(0, T ; \mathbb{X}(\Omega))
$$

This completes the proof. 
Theorem 3.2. The weak limit $\tilde{\mathcal{U}}(t)=(\tilde{\mathbf{u}}(t), \tilde{\varphi}(t), \tilde{\zeta}(t), \tilde{\theta}(t))$ is the solution to the limit variational problem:

$$
\left\{\begin{array}{l}
\text { Find } \widetilde{\mathcal{U}}(t) \in \mathbf{V}_{K L}(\Omega) \times H^{1}\left(\Omega, \Gamma_{0}\right) \times \mathbb{X}(\Omega) \times H^{1}\left(\Omega, \Gamma_{0}\right), t \in(0, T) \text { such that } \\
\widetilde{A}_{1}(\widetilde{\mathcal{U}}(t), \mathcal{V})=\widetilde{L}_{1}(\mathcal{V}), \text { for all } \mathcal{V} \in \mathbf{V}_{K L}(\Omega) \times H^{1}\left(\Omega, \Gamma_{0}\right) \times \mathbb{X}_{0}(\Omega) \times H^{1}\left(\Omega, \Gamma_{0}\right), \\
\tilde{\zeta}=\zeta^{ \pm} \text {on } \Gamma_{ \pm},
\end{array}\right.
$$

where

$$
\begin{aligned}
& \widetilde{A}_{1}(\widetilde{\mathcal{U}}(t), \mathcal{V}):=\int_{\Omega}\{\left(\widetilde{C}_{\alpha \beta \sigma \tau}^{1} e_{\sigma \tau}(\tilde{\mathbf{u}}(t))+\widetilde{P}_{\sigma \alpha \beta}^{1} \partial_{\sigma} \tilde{\varphi}(t)+\widetilde{R}_{3 \alpha \beta}^{1} \partial_{3} \tilde{\zeta}(t)-\widetilde{\beta}_{\alpha \beta}^{1} \tilde{\theta}(t)\right) e_{\alpha \beta}(\mathbf{v})+ \\
&+\left(-\widetilde{P}_{\alpha \sigma \tau}^{1} e_{\sigma \tau}(\tilde{\mathbf{u}}(t))+\widetilde{X}_{\alpha \beta}^{1} \partial_{\beta} \tilde{\varphi}(t)+\widetilde{\alpha}_{\alpha 3}^{1} \partial_{3} \tilde{\zeta}(t)-\widetilde{p}_{\alpha}^{1} \tilde{\theta}(t)\right) \partial_{\alpha} \psi+ \\
&+\left(-\widetilde{R}_{3 \alpha \beta}^{1} e_{\alpha \beta}(\tilde{\mathbf{u}}(t))+\widetilde{\alpha}_{\alpha 3}^{1} \partial_{\alpha} \tilde{\varphi}(t)+\widetilde{M}_{33}^{1} \partial_{3} \tilde{\zeta}(t)-\widetilde{m}_{3}^{1} \tilde{\theta}(t)\right) \partial_{3} \xi+ \\
&+\left(\widetilde{\beta}_{\alpha \beta}^{1} e_{\alpha \beta}(\dot{\tilde{\mathbf{u}}}(t))-\widetilde{m}_{3}^{1} \partial_{3} \dot{\tilde{\zeta}}(t)-\widetilde{p}_{\alpha}^{1} \partial_{\alpha} \dot{\tilde{\varphi}}(t)+\widetilde{c}_{v}^{1} \dot{\tilde{\theta}}(t)\right) \eta+ \\
&\left.+\widetilde{K}_{\alpha \beta}^{1} \partial_{\beta} \tilde{\theta}(t) \partial_{\alpha} \eta+\ddot{\tilde{u}}_{i}(t) v_{i}\right\} d x \\
& \widetilde{L}_{1}(\mathcal{V}):=(\mathbf{f}, \mathbf{v})+(\mathbf{g}, \mathbf{v})_{\mathbf{L}^{2}(\widehat{\Gamma})}+(r, \eta)-(\varrho, \eta)_{L^{2}(\widehat{\Gamma})}+\left(\rho_{e}, \psi\right)-(d, \psi)_{L^{2}(\widehat{\Gamma})} .
\end{aligned}
$$

The reduced magneto-electro-thermo-elastic coefficients $\widetilde{C}_{\alpha \beta \sigma \tau}^{1}, \widetilde{X}_{\alpha \beta}^{1}, \widetilde{K}_{\alpha \beta}^{1}, \widetilde{P}_{\sigma \alpha \beta}^{1}, \widetilde{\beta}_{\alpha \beta}^{1}$, $\widetilde{p}_{\alpha}^{1} \widetilde{m}_{3}^{1}, \widetilde{R}_{3 \alpha \beta}^{1}$ and $\widetilde{\alpha}_{\alpha 3}^{1}$ are listed in Appendix 1 .

Proof. For the sake of clarity the proof is split into three parts.

(i) By the definition of $\kappa_{i j}(\varepsilon), \bar{\chi}_{i}(\varepsilon), \tau_{i}(\varepsilon)$ and $\gamma_{i}(\varepsilon)$, and thanks to the results of Theorem 3.1, there exists two constants $C_{M}$ and $C_{K}$ such that

$$
\begin{aligned}
& \left|e_{\alpha \beta}(\mathbf{u}(\varepsilon))\right|_{0, \Omega} \leq C_{M} e^{C_{K} T},\left|e_{\alpha 3}(\mathbf{u}(\varepsilon))\right|_{0, \Omega} \leq \varepsilon C_{M} e^{C_{K} T},\left|e_{33}(\mathbf{u}(\varepsilon))\right|_{0, \Omega} \leq \varepsilon^{2} C_{M} e^{C_{K} T} \\
& \left|\partial_{\alpha} \zeta(\varepsilon)\right|_{0, \Omega} \leq \frac{1}{\varepsilon} C_{M} e^{C_{K} T}, \quad\left|\partial_{3} \zeta(\varepsilon)\right|_{0, \Omega} \leq C_{M} e^{C_{K} T} \\
& \left|\partial_{\alpha} \varphi(\varepsilon)\right|_{0, \Omega} \leq C_{M} e^{C_{K} T}, \quad\left|\partial_{3} \varphi(\varepsilon)\right|_{0, \Omega} \leq \varepsilon C_{M} e^{C_{K} T} \\
& \left|\partial_{\alpha} \theta(\varepsilon)\right|_{0, \Omega} \leq C_{M} e^{C_{K} T}, \quad\left|\partial_{3} \theta(\varepsilon)\right|_{0, \Omega} \leq \varepsilon C_{M} e^{C_{K} T}
\end{aligned}
$$

From the first set of inequalities $(3.5)_{1}$, we get that $e_{i 3}(\mathbf{u}(\varepsilon)(t)) \rightarrow 0$ in $L^{2}(\Omega)$ for almost every $t \in(0, T)$. Also, as $\mathbf{u}(\varepsilon)(t) \rightarrow \tilde{\mathbf{u}}(t)$ in $\mathbf{H}^{1}(\Omega)$, we have that $e_{i 3}(\mathbf{u}(\varepsilon)(t)) \rightarrow e_{i 3}(\tilde{\mathbf{u}}(t))$ and so $e_{i 3}(\tilde{\mathbf{u}}(t))=0$ by uniqueness of the limit. This implies that $\partial_{3} \tilde{u}_{3}=0$, i.e., $\tilde{u}_{3}\left(\tilde{x}, x_{3}\right)=$ $\tilde{u}_{3}(\tilde{x})$ is independent of $x_{3}$. We also have that $\partial_{3} \tilde{u}_{\alpha}=-\partial_{\alpha} \tilde{u}_{3}$, i.e., $\tilde{u}_{\alpha}\left(\tilde{x}, x_{3}\right)=\tilde{u}_{\alpha}(\tilde{x})-$ $x_{3} \partial_{\alpha} \tilde{u}_{3}(\tilde{x})$. Consequently, $\tilde{\mathbf{u}}(t) \in \mathbf{V}_{K L}(\Omega)$. Moreover, we obtain that $e_{\alpha \beta}(\mathbf{u}(\varepsilon)(t)) \rightarrow$ $\kappa_{\alpha \beta}(t)=e_{\alpha \beta}(\tilde{\mathbf{u}}(t))$ in $L^{2}(\Omega), \frac{1}{\varepsilon} e_{\alpha 3}(\mathbf{u}(\varepsilon)(t)) \rightarrow \kappa_{\alpha 3}(t)$ in $L^{2}(\Omega), \frac{1}{\varepsilon} e_{33}(\mathbf{u}(\varepsilon)(t)) \rightarrow 0$ in $L^{2}(\Omega)$ and, also, $\frac{1}{\varepsilon^{2}} e_{33}(\mathbf{u}(\varepsilon)(t)) \rightarrow \kappa_{33}(t)$ in $L^{2}(\Omega)$.

From the second set of inequalities $(3.5)_{2}$, we have that $\varepsilon \partial_{\alpha} \zeta(\varepsilon)(t) \rightarrow 0$ in $L^{2}(\Omega)$ and $\partial_{3} \zeta(\varepsilon)(t) \rightarrow \partial_{3} \tilde{\zeta}(t)$ in $L^{2}(\Omega)$.

From the last sets of inequalities $(3.5)_{3,4}$, since $\varphi(\varepsilon)(t) \rightarrow \tilde{\varphi}(t)$ in $H^{1}(\Omega)$, we infer that $\partial_{\alpha} \varphi(\varepsilon)(t) \rightarrow \tilde{\tau}_{\alpha}(t)=\partial_{\alpha} \tilde{\varphi}(t)$ in $L^{2}(\Omega)$ and, also, $\partial_{3} \varphi(\varepsilon)(t) \rightarrow 0$ in $L^{2}(\Omega)$, i.e., $\tilde{\varphi}(t)=\tilde{\varphi}(\tilde{x})(t)$ is independent of $x_{3}$. Besides, $\frac{1}{\varepsilon} \partial_{3} \varphi(\varepsilon)(t) \rightarrow \tau_{3}(t)$ in $L^{2}(\Omega)$. Similarly, since $\theta(\varepsilon)(t) \rightarrow \tilde{\theta}(t)$ in $H^{1}(\Omega)$, we obtain that $\partial_{\alpha} \theta(\varepsilon)(t) \rightarrow \tilde{\gamma}_{\alpha}(t)=\partial_{\alpha} \tilde{\theta}(t)$ in $L^{2}(\Omega)$, $\partial_{3} \theta(\varepsilon)(t) \rightarrow 0$ in $L^{2}(\Omega)$, i.e., $\tilde{\theta}(t)=\tilde{\theta}(\tilde{x})(t)$ and, finally, $\frac{1}{\varepsilon} \partial_{3} \theta(\varepsilon)(t) \rightarrow \gamma_{3}(t)$ in $L^{2}(\Omega)$.

(ii) Computations of $\kappa_{i 3}, \tau_{3}$ and $\gamma_{3}$. Let us multiply problem (2.5) by $\varepsilon^{2}$ and let $\varepsilon$ tend to zero. We get the following equation:

$$
C_{3333} \kappa_{33}+2 C_{\alpha 333} \kappa_{\alpha 3}+P_{333} \tau_{3}+C_{\alpha \beta 33} e_{\alpha \beta}(\tilde{\mathbf{u}})+P_{\alpha 33} \partial_{\alpha} \tilde{\varphi}+R_{333} \partial_{3} \tilde{\zeta}-\beta_{33} \tilde{\theta}=0 .
$$


By multiplying problem (2.5) by $\varepsilon$, choosing test functions $v_{3}=\psi=\eta=\xi=0$ and letting $\varepsilon$ tend to zero, we have that

$$
C_{\alpha 333} \kappa_{33}+2 C_{\alpha 3 \beta 3} \kappa_{\beta 3}+P_{3 \alpha 3} \tau_{3}+C_{\sigma \beta \alpha 3} e_{\sigma \beta}(\tilde{\mathbf{u}})+P_{\sigma \alpha 3} \partial_{\sigma} \tilde{\varphi}+R_{3 \alpha 3} \partial_{3} \tilde{\zeta}-\beta_{\alpha 3} \tilde{\theta}=0 .
$$

Similarly, by multiplying problem (2.5) by $\varepsilon$ and choosing test functions $v_{i}=\eta=\xi=0$, when $\varepsilon$ tends to zero, we find

$$
-P_{333} \kappa_{33}-2 P_{3 \alpha 3} \kappa_{\alpha 3}+X_{33} \tau_{3}-P_{3 \alpha \beta} e_{\alpha \beta}(\tilde{\mathbf{u}})+X_{\alpha 3} \partial_{\alpha} \tilde{\varphi}+\alpha_{33} \partial_{3} \tilde{\zeta}-p_{3} \tilde{\theta}=0 .
$$

Finally, if we multiply by $\varepsilon$ and choose test functions $v_{i}=\psi=\xi=0$, we obtain the last equation

$$
K_{33} \gamma_{3}+K_{\alpha 3} \partial_{\alpha} \tilde{\theta}=0 .
$$

By combining the whole set of equations above we are now in a position to characterize $\kappa_{i 3}, \tau_{3}$ and $\gamma_{3}$. Let $\mathbf{l}^{1}=\left(l_{i}^{1}\right)$ be the vector whose components are defined by $l_{\alpha}^{1}:=2 \kappa_{\alpha 3}$ and $l_{3}^{1}:=\kappa_{33}$, and $\left(d_{i j}\right):=\left(C_{i 3 j 3}\right)^{-1}$, then

$$
\begin{aligned}
l_{i}^{1}= & -d_{i j}\left\{\left(C_{\alpha \beta j 3}+k^{\prime} P_{3 j 3} P_{3 \alpha \beta}^{\prime}\right) e_{\alpha \beta}(\tilde{\mathbf{u}})+\left(P_{\alpha j 3}-k^{\prime} P_{3 j 3} X_{\alpha 3}^{\prime}\right) \partial_{\alpha} \tilde{\varphi}+\right. \\
& \left.+\left(R_{3 j 3}-k^{\prime} P_{3 j 3} \alpha_{33}^{\prime}\right) \partial_{3} \tilde{\zeta}-\left(\beta_{j 3}-k^{\prime} P_{3 j 3} p_{3}^{\prime}\right) \tilde{\theta}\right\} \\
\tau_{3}= & k^{\prime}\left(P_{3 \alpha \beta}^{\prime} e_{\alpha \beta}(\tilde{\mathbf{u}})-X_{\alpha 3}^{\prime} \partial_{\alpha} \tilde{\varphi}-\alpha_{33}^{\prime} \partial_{3} \tilde{\zeta}+p_{3}^{\prime} \tilde{\theta}\right) \\
\gamma_{3}= & -K_{\alpha 3}^{\prime} \partial_{\alpha} \tilde{\theta} .
\end{aligned}
$$

Coefficients $k^{\prime}, P_{3 \alpha \beta}^{\prime}, X_{\alpha 3}^{\prime}, \alpha_{33}^{\prime}, p_{3}^{\prime}$ and $K_{\alpha 3}^{\prime}$ are defined in Appendix 1 .

(iii) Definition of the limit problem. We let test functions be $\mathcal{V}=(\mathbf{v}, \psi, \xi, \eta) \in \mathbf{V}_{K L}(\Omega) \times$ $H^{1}\left(\Omega, \Gamma_{0}\right) \times \mathbb{X}_{0}(\Omega) \times H^{1}\left(\Omega, \Gamma_{0}\right)$ in problem $(2.5)$ and let $\varepsilon \rightarrow 0$, by substituting expressions (3.6), we obtain, as customary, the limit evolution problem

$$
\left\{\begin{aligned}
\text { Find } & \widetilde{\mathcal{U}}(t) \in \mathbf{V}_{K L}(\Omega) \times H^{1}\left(\Omega, \Gamma_{0}\right) \times \mathbb{X}(\Omega) \times H^{1}\left(\Omega, \Gamma_{0}\right), t \in(0, T) \text { such that } \\
\int_{\Omega}\{ & \left(\widetilde{C}_{\alpha \beta \sigma \tau}^{1} e_{\sigma \tau}(\tilde{\mathbf{u}}(t))+\widetilde{P}_{\sigma \alpha \beta}^{1} \partial_{\sigma} \tilde{\varphi}(t)+\widetilde{R}_{3 \alpha \beta}^{1} \partial_{3} \tilde{\zeta}(t)-\widetilde{\beta}_{\alpha \beta}^{1} \tilde{\theta}(t)\right) e_{\alpha \beta}(\mathbf{v})+ \\
& +\left(-\widetilde{P}_{\alpha \sigma \tau}^{1} e_{\sigma \tau}(\tilde{\mathbf{u}}(t))+\widetilde{X}_{\alpha \beta}^{1} \partial_{\beta} \tilde{\varphi}(t)+\widetilde{\alpha}_{\alpha 3}^{1} \partial_{3} \tilde{\zeta}(t)-\widetilde{p}_{\alpha}^{1} \tilde{\theta}(t)\right) \partial_{\alpha} \psi+ \\
& +\left(-\widetilde{R}_{3 \alpha \beta}^{1} e_{\alpha \beta}(\tilde{\mathbf{u}}(t))+\widetilde{\alpha}_{\alpha 3}^{1} \partial_{\alpha} \tilde{\varphi}(t)+\widetilde{M}_{33}^{1} \partial_{3} \tilde{\zeta}(t)-\widetilde{m}_{3}^{1} \tilde{\theta}(t)\right) \partial_{3} \xi+ \\
& +\left(\widetilde{\beta}_{\alpha \beta}^{1} e_{\alpha \beta}(\dot{\tilde{\mathbf{u}}}(t))-\widetilde{m}_{3}^{1} \partial_{3} \dot{\tilde{\zeta}}(t)-\widetilde{p}_{\alpha}^{1} \partial_{\alpha} \dot{\tilde{\varphi}}(t)+\widetilde{c}_{v}^{1} \dot{\tilde{\theta}}(t)\right) \eta+ \\
& \left.+\widetilde{K}_{\alpha \beta}^{1} \partial_{\beta} \tilde{\theta}(t) \partial_{\alpha} \eta+\rho \ddot{\tilde{u}}_{i}(t) v_{i}\right\} d x=\widetilde{L}_{1}(\mathcal{V}), \\
\tilde{\zeta}= & \zeta^{ \pm} \text {on } \Gamma_{ \pm}, \text {for all } \mathcal{V} \in \mathbf{V}_{K L}(\Omega) \times H^{1}\left(\Omega, \Gamma_{0}\right) \times \mathbb{X}_{0}(\Omega) \times H^{1}\left(\Omega, \Gamma_{0}\right)
\end{aligned}\right.
$$

Problem (3.7) is formally equivalent to (3.4), presented in the statement of Theorem 3.2.

\subsubsection{The limit evolution problem}

In this subsection we present the variational and differential formulations of the evolution problem for a sensor-actuator magneto-electro-thermo-elastic plate. The primary unknowns of the limit problem are collected into the limit state $\widetilde{\mathcal{U}}(t)=$ $(\tilde{\mathbf{u}}(t), \tilde{\varphi}(t), \tilde{\zeta}(t), \tilde{\theta}(t)) \in \mathbf{V}_{K L}(\Omega) \times H^{1}\left(\Omega, \Gamma_{0}\right) \times \mathbb{X}(\Omega) \times H^{1}\left(\Omega, \Gamma_{0}\right)$, whose components take the following form:

$$
\begin{aligned}
& \tilde{u}_{\alpha}\left(\tilde{x}, x_{3}\right)=u_{\alpha}(\tilde{x})-x_{3} \partial_{\alpha} u_{3}(\tilde{x}), \mathbf{u}_{H}:=\left(u_{\alpha}\right), \\
& \tilde{u}_{3}\left(\tilde{x}, x_{3}\right)=u_{3}(\tilde{x}), \\
& \tilde{\varphi}\left(\tilde{x}, x_{3}\right)=\phi(\tilde{x}), \\
& \tilde{\theta}\left(\tilde{x}, x_{3}\right)=\vartheta(\tilde{x}) .
\end{aligned}
$$


In the case of a homogeneous material, the reduced magneto-electro-thermo-elastic coefficients $\widetilde{C}_{\alpha \beta \sigma \tau}^{1}, \widetilde{X}_{\alpha \beta}^{1}, \widetilde{K}_{\alpha \beta}^{1}, \widetilde{P}_{\sigma \alpha \beta}^{1}, \widetilde{\beta}_{\alpha \beta}^{1}, \widetilde{p}_{\alpha}^{1} \widetilde{m}_{3}^{1}, \widetilde{R}_{3 \alpha \beta}^{1}$ and $\widetilde{\alpha}_{\alpha 3}^{1}$ are constant functions and, thus, the limit evolution problem decouples into two variational subproblems, namely, the flexural problem, which gives $u_{3}$, and the thermo-piezoelectric membrane problem, which gives the triplet $\left(\mathbf{u}_{H}, \phi, \vartheta\right)$. Moreover, we can characterize explicitly the limit magnetic potential $\tilde{\zeta}$ as a second order polynomial function of $x_{3}$.

Indeed, by choosing test functions $\mathcal{V}=(\mathbf{0}, 0, \xi, 0)$ in $(3.7)$, after an integration by parts along $x_{3}$, we get the expression of the limit magnetic potential $\tilde{\zeta}$ :

$$
\tilde{\zeta}\left(\tilde{x}, x_{3}\right)=\sum_{k=0}^{2} z^{k}(\tilde{x}) x_{3}^{k}
$$

where

$$
z^{0}=\langle\zeta\rangle+\frac{h^{2} \widetilde{R}_{3 \alpha \beta}^{1}}{2 \widetilde{M}_{33}^{1}} \partial_{\alpha \beta} u_{3}, \quad z^{1}=\frac{\llbracket \zeta \rrbracket}{2 h}, \quad z^{2}=-\frac{\widetilde{R}_{3 \alpha \beta}^{1}}{2 \widetilde{M}_{33}^{1}} \partial_{\alpha \beta} u_{3}
$$

with $\langle\zeta\rangle:=\frac{\zeta^{+}+\zeta^{-}}{2}$ and $\llbracket \zeta \rrbracket:=\zeta^{+}-\zeta^{-}$representing, respectively, the mean value and the jump function between the top and bottom boundary values of $\zeta$. As the careful reader can notice, the limit magnetic potential becomes a known function depending on the transversal displacement $u_{3}$ of the plate and on the values of the magnetic potentials $\zeta^{ \pm}$, applied on the upper and lower surfaces $\Gamma_{ \pm}$.

By virtue of the characterization (3.9) of the limit magnetic potential $\tilde{\zeta}$, we can now rewrite the limit evolution problem. After an integration by parts along $x_{3}$, we obtain a two-dimensional problem defined over the middle surface $\omega$ of the plate:

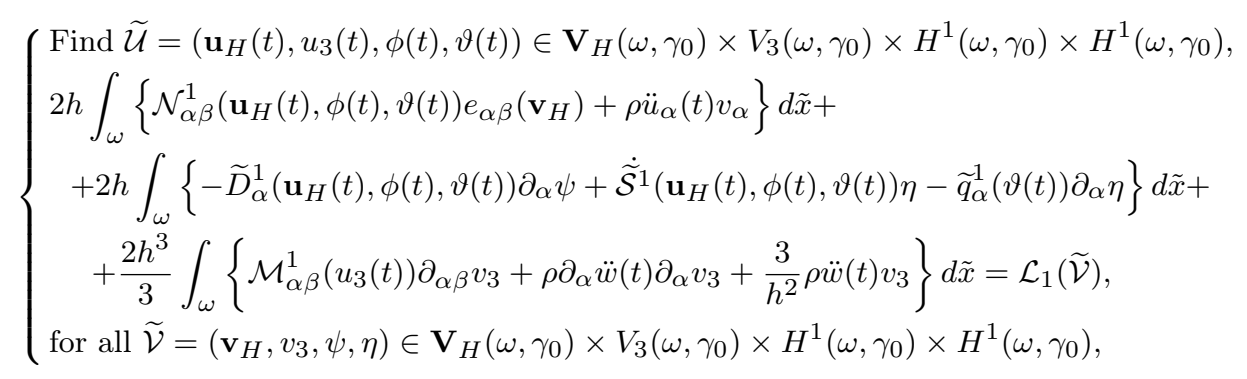

with

$$
\begin{aligned}
\mathcal{L}_{1}(\widetilde{\mathcal{V}}):= & \int_{\omega}\left\{\widetilde{f}_{i} v_{i}-m_{\alpha} \partial_{\alpha} v_{3}+\widetilde{\rho}_{e} \psi+\widetilde{r} \eta\right\} d \tilde{x}+\int_{\gamma_{1}}\left\{\widetilde{g}_{i} v_{i}-n_{\alpha} \partial_{\alpha} v_{3}-\widetilde{d} \psi-\widetilde{\varrho} \eta\right\} d \gamma- \\
& -\int_{\omega}\left\{\widetilde{R}_{3 \alpha \beta}^{1} \llbracket \zeta \rrbracket e_{\alpha \beta}\left(\mathbf{v}_{H}\right)+\widetilde{\alpha}_{\alpha 3}^{1} \llbracket \zeta \rrbracket \partial_{\alpha} \psi-\widetilde{m}_{3}^{1} \llbracket \dot{\zeta} \rrbracket \eta\right\} d \tilde{x} .
\end{aligned}
$$

The initial conditions are given by

$$
\left\{\begin{array}{l}
\tilde{\mathbf{u}}(0)=\tilde{\mathbf{u}}_{0}=\left(u_{\alpha, 0}-x_{3} \partial_{\alpha} u_{3,0}, u_{3,0}\right) \\
\dot{\tilde{\mathbf{u}}}(0)=\tilde{\mathbf{u}}_{1}=\left(u_{\alpha, 1}-x_{3} \partial_{\alpha} u_{3,1}, u_{3,1}\right) \\
\tilde{\theta}(0)=\tilde{\theta}_{0}=\vartheta_{0}
\end{array}\right.
$$

$\left(\mathcal{N}_{\alpha \beta}^{1}\right),\left(\mathcal{M}_{\alpha \beta}^{1}\right),\left(\widetilde{D}_{\alpha}^{1}\right), \widetilde{\mathcal{S}}^{1}$ and $\left(\widetilde{q}_{\alpha}^{1}\right)$ represent, respectively, the membrane stress tensor, the moment tensor, the reduced electric displacement vector, the reduced thermodynamic 
entropy and the reduced heat flow vector of the plate, whose components are defined by the constitutive laws below:

$$
\left\{\begin{array}{l}
\mathcal{N}_{\alpha \beta}^{1}:=\widetilde{C}_{\alpha \beta \sigma \tau}^{1} e_{\sigma \tau}\left(\mathbf{u}_{H}\right)+\widetilde{P}_{\sigma \alpha \beta}^{1} \partial_{\sigma} \phi-\widetilde{\beta}_{\alpha \beta}^{1} \vartheta \\
\widetilde{D}_{\alpha}^{1}:=\widetilde{P}_{\alpha \sigma \tau}^{1} e_{\sigma \tau}\left(\mathbf{u}_{H}\right)-\widetilde{X}_{\alpha \beta}^{1} \partial_{\beta} \phi+\widetilde{p}_{\alpha}^{1} \vartheta \\
\widetilde{\mathcal{S}}^{1}:=\widetilde{\beta}_{\alpha \beta}^{1} e_{\alpha \beta}\left(\mathbf{u}_{H}\right)-\widetilde{p}_{\alpha}^{1} \partial_{\alpha} \phi+\widetilde{c}_{v}^{1} \vartheta \\
\mathcal{M}_{\alpha \beta}^{1}:=\widetilde{A}_{\alpha \beta \sigma \tau}^{1} \partial_{\sigma \tau} u_{3} \\
\widetilde{q}_{\alpha}^{1}:=-\widetilde{K}_{\alpha \beta}^{1} \partial_{\beta} \vartheta
\end{array}\right.
$$

where $\widetilde{A}_{\alpha \beta \sigma \tau}^{1}:=\widetilde{C}_{\alpha \beta \sigma \tau}^{1}+\frac{\widetilde{R}_{3 \alpha \beta}^{1} \widetilde{R}_{3 \sigma \tau}^{1}}{\widetilde{M}_{33}^{1}}$.

Moreover, the two-dimensional applied thermo-electro-mechanical loads are

$$
\begin{aligned}
& \widetilde{f}_{i}(t):=\int_{-h}^{h} f_{i}(t) d x_{3}+g_{i}^{+}(t)+g_{i}^{-}(t), \\
& m_{\alpha}(t):=\int_{-h}^{h} x_{3} f_{\alpha}(t) d x_{3}+h\left(g_{\alpha}^{+}(t)-g_{\alpha}^{-}(t)\right), \\
& \widetilde{g}_{i}(t):=\int_{-h}^{h} g_{i}(t) d x_{3}, \quad n_{\alpha}(t):=\int_{-h}^{h} x_{3} g_{\alpha}(t) d x_{3}, \\
& \widetilde{\rho}_{e}(t):=\int_{-h}^{h} \rho_{e}(t) d x_{3}-d^{+}(t)-d^{-}(t), \quad \widetilde{d}(t):=\int_{-h}^{h} d(t) d x_{3}, \\
& \widetilde{r}(t):=\int_{-h}^{h} r(t) d x_{3}-\varrho^{+}(t)-\varrho^{-}(t), \quad \widetilde{\varrho}(t):=\int_{-h}^{h} \varrho(t) d x_{3},
\end{aligned}
$$

where $v^{ \pm}:=\left.v\right|_{\Gamma_{ \pm}}=v(\tilde{x}, \pm h)$ denotes the restriction of $v$ to $\Gamma_{ \pm}$.

This problem can be split into two two-dimensional decoupled problems: the flexural problem and the thermo-piezoelectric membrane problem. The flexural problem is:

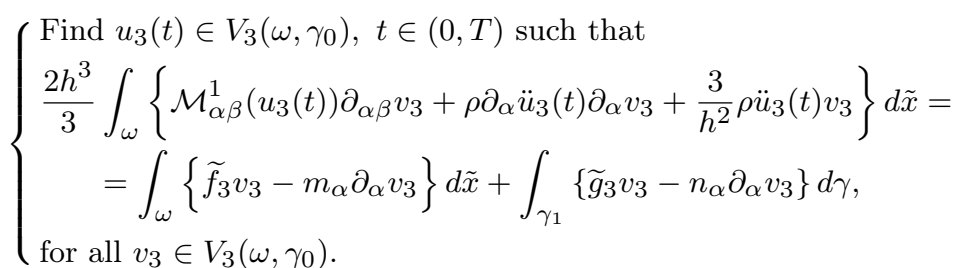

The two-dimensional thermo-piezoelectric membrane problem takes the following form

$$
\left\{\begin{array}{l}
\text { Find }\left(\mathbf{u}_{H}(t), \phi(t), \vartheta(t)\right), \in \mathbf{V}_{H}\left(\omega, \gamma_{0}\right) \times H^{1}\left(\omega, \gamma_{0}\right) \times H^{1}\left(\omega, \gamma_{0}\right), t \in(0, T) \text { such that } \\
2 h \int_{\omega}\left\{\mathcal{N}_{\alpha \beta}^{1}\left(\mathbf{u}_{H}(t), \phi(t), \vartheta(t)\right) e_{\alpha \beta}\left(\mathbf{v}_{H}\right)+\rho \ddot{u}_{\alpha}(t) v_{\alpha}\right\} d \tilde{x}+ \\
+2 h \int_{\omega}\left\{-\widetilde{D}_{\alpha}^{1}\left(\mathbf{u}_{H}(t), \phi(t), \vartheta(t)\right) \partial_{\alpha} \psi+\dot{\mathcal{S}}^{1}\left(\mathbf{u}_{H}(t), \phi(t), \vartheta(t)\right) \eta-\widetilde{q}_{\alpha}^{1}(\vartheta(t)) \partial_{\alpha} \eta\right\} d \tilde{x}= \\
=\int_{\omega}\left\{\widetilde{f}_{\alpha} v_{\alpha}+\widetilde{\rho}_{e} \psi-\widetilde{R}_{3 \alpha \beta} \llbracket \zeta \rrbracket e_{\alpha \beta}\left(\mathbf{v}_{H}\right)-\widetilde{\alpha}_{\alpha 3} \llbracket \zeta \rrbracket \partial_{\alpha} \psi+\left(\widetilde{r}+\widetilde{m}_{3} \llbracket \dot{\zeta} \rrbracket\right) \eta\right\} d \tilde{x}+ \\
\quad+\int_{\gamma_{1}}\left\{\widetilde{g}_{\alpha} v_{\alpha}-\widetilde{d} \psi-\widetilde{\varrho} \eta\right\} d \gamma, \\
\text { for all }\left(\mathbf{v}_{H}, \psi, \eta\right) \in \mathbf{V}_{H}\left(\omega, \gamma_{0}\right) \times H^{1}\left(\omega, \gamma_{0}\right) \times H^{1}\left(\omega, \gamma_{0}\right) .
\end{array}\right.
$$

We are now in a position to write the decoupled flexural and thermo-piezoelectric membrane problems into their differential form by using Green's formulae on $\omega$. 
The transversal displacement $u_{3}$ solves the following flexural differential problem:

$$
\left\{\begin{array}{lr}
\text { Field equation: } & \text { in } \omega \times(0, T), \\
\frac{2 h^{3}}{3} \partial_{\alpha \beta} \mathcal{M}_{\alpha \beta}^{1}-\frac{2 h^{3}}{3} \rho \partial_{\alpha \alpha} \ddot{u}_{3}+2 h \rho \ddot{u}_{3}=\mathcal{F}_{3} & \text { in } \omega \times(0, T), \\
\text { Initial conditions: } & \\
u_{3}(0)=u_{3,0}, \dot{u}_{3}(0)=u_{3,1} & \\
\text { Boundary conditions: } & \text { on } \gamma_{1} \times(0, T), \\
\frac{2 h^{3}}{3}\left\{\rho \partial_{\alpha} \ddot{u}_{3} \nu_{\alpha}-\partial_{\alpha} \mathcal{M}_{\alpha \beta}^{1} \nu_{\beta}-\partial_{\tau}\left(\mathcal{M}_{\alpha \beta}^{1} \nu_{\alpha} \tau_{\beta}\right)\right\}=\mathcal{G}_{3} \\
\mathcal{M}_{\alpha \beta}^{1} \nu_{\alpha} \nu_{\beta}=0 & \text { on } \gamma_{1} \times(0, T), \\
u_{3}=\partial_{\nu} u_{3}=0 & \text { on } \gamma_{0} \times(0, T),
\end{array}\right.
$$

where $\mathcal{F}_{3}:=\widetilde{f}_{3}+\partial_{\alpha} m_{\alpha}$ and $\mathcal{G}_{3}:=\widetilde{g}_{3}-m_{\alpha} \nu_{\alpha}+\partial_{\alpha} n_{\alpha}$.

The thermo-electro-mechanical state $\left(\mathbf{u}_{H}(t), \phi(t), \vartheta(t)\right)$ solves the following thermopiezoelectric membrane differential problem:

$$
\begin{cases}\text { Field equations: } & \\ 2 h\left(\rho \ddot{u}_{\alpha}-\partial_{\beta} \mathcal{N}_{\alpha \beta}^{1}\right)=\widetilde{f}_{\alpha}+\widetilde{R}_{3 \alpha \beta}^{1} \llbracket \partial_{\beta} \zeta \rrbracket & \text { in } \omega \times(0, T), \\ 2 h \partial_{\alpha} \widetilde{D}_{\alpha}^{1}=\widetilde{\rho}_{e}+\widetilde{\alpha}_{\alpha 3}^{1} \llbracket \partial_{\alpha} \zeta \rrbracket & \text { in } \omega \times(0, T), \\ 2 h\left(\dot{\mathcal{\mathcal { S }}}^{1}+\partial_{\alpha} \widetilde{q}_{\alpha}^{1}\right)=\widetilde{r}+\widetilde{m}_{3}^{1} \llbracket \dot{\zeta} \rrbracket & \text { in } \omega \times(0, T), \\ \text { Initial conditions: } & \\ u_{\alpha}(0)=u_{\alpha, 0}, \dot{u}_{\alpha}(0)=u_{\alpha, 1}, \vartheta(0)=\vartheta_{0} & \text { in } \omega \times(0, T), \\ \text { Boundary conditions: } & \\ 2 h \mathcal{N}_{\alpha \beta}^{1} \nu_{\beta}=\widetilde{g}_{\alpha}-\widetilde{R}_{3 \alpha \beta}^{1} \nu_{\beta} \llbracket \zeta \rrbracket & \text { on } \gamma_{1} \times(0, T), \\ 2 h \widetilde{D}_{\alpha}^{1} \nu_{\alpha}=\widetilde{d}+\widetilde{\alpha}_{\alpha 3}^{1} \nu_{\alpha} \llbracket \zeta \rrbracket & \text { on } \gamma_{1} \times(0, T), \\ 2 h \widetilde{q}_{\alpha}^{1} \nu_{\alpha}=\widetilde{\varrho} & \text { on } \gamma_{1} \times(0, T), \\ u_{\alpha}=\phi=\vartheta=0 & \text { on } \gamma_{0} \times(0, T) .\end{cases}
$$

It is important to remark that the information regarding the piezomagnetic behavior of the plate is now contained in the source terms appearing on the right-hand side of the equations, depending on the jump of the applied magnetic potentials at the upper and lower faces of the plate.

\subsection{The Actuator Model}

Theorem 3.3. Under assumption (3.2), the sequence $\{\mathcal{U}(\varepsilon)\}_{\varepsilon>0}$ weakly converges to the limit $\tilde{\mathcal{U}}:=(\tilde{\mathbf{u}}, \tilde{\varphi}, \tilde{\zeta}, \tilde{\theta})$ in the space $L^{2}\left(0, T ; \mathbf{H}^{1}(\Omega)\right) \times L^{2}(0, T ; \mathbb{X}(\Omega)) \times L^{2}(0, T ; \mathbb{X}(\Omega)) \times$ $L^{2}\left(0, T ; H^{1}(\Omega)\right)$.

Theorem 3.4. The weak limit $\tilde{\mathcal{U}}(t)=(\tilde{\mathbf{u}}(t), \tilde{\varphi}(t), \tilde{\zeta}(t), \tilde{\theta}(t))$ is the solution to the limit variational problem:

$$
\left\{\begin{array}{l}
\text { Find } \widetilde{\mathcal{U}}(t) \in \mathbf{V}_{K L}(\Omega) \times \mathbb{X}(\Omega) \times \mathbb{X}(\Omega) \times H^{1}\left(\Omega, \Gamma_{0}\right), t \in(0, T) \text { such that } \\
\widetilde{A}_{3}(\widetilde{\mathcal{U}}(t), \mathcal{V})=\widetilde{L}_{3}(\mathcal{V}), \text { for all } \mathcal{V} \in \mathbf{V}_{K L}(\Omega) \times \mathbb{X}_{0}(\Omega) \times \mathbb{X}_{0}(\Omega) \times H^{1}\left(\Omega, \Gamma_{0}\right) \\
\tilde{\varphi}=\varphi^{ \pm}, \tilde{\zeta}=\zeta^{ \pm} \text {on } \Gamma_{ \pm},
\end{array}\right.
$$


where

$$
\begin{aligned}
& \widetilde{A}_{3}(\widetilde{\mathcal{U}}(t), \mathcal{V}):=\int_{\Omega}\{\left(\widetilde{C}_{\alpha \beta \sigma \tau}^{3} e_{\sigma \tau}(\tilde{\mathbf{u}}(t))+\widetilde{P}_{3 \alpha \beta}^{3} \partial_{3} \tilde{\varphi}(t)+\widetilde{R}_{3 \alpha \beta}^{3} \partial_{3} \tilde{\zeta}(t)-\widetilde{\beta}_{\alpha \beta}^{3} \tilde{\theta}(t)\right) e_{\alpha \beta}(\mathbf{v})+ \\
&+\left(-\widetilde{P}_{3 \alpha \beta}^{3} e_{\alpha \beta}(\tilde{\mathbf{u}}(t))+\widetilde{X}_{33}^{3} \partial_{3} \tilde{\varphi}(t)+\widetilde{\alpha}_{33}^{3} \partial_{3} \tilde{\zeta}(t)-\widetilde{p}_{3}^{3} \tilde{\theta}(t)\right) \partial_{3} \psi+ \\
&+\left(-\widetilde{R}_{3 \alpha \beta}^{3} e_{\alpha \beta}(\tilde{\mathbf{u}}(t))+\widetilde{\alpha}_{33}^{3} \partial_{3} \tilde{\varphi}(t)+\widetilde{M}_{33}^{3} \partial_{3} \tilde{\zeta}(t)-\widetilde{m}_{3}^{3} \tilde{\theta}(t)\right) \partial_{3} \xi+ \\
&+\left(\widetilde{\beta}_{\alpha \beta}^{3} e_{\alpha \beta}(\dot{\tilde{\mathbf{u}}}(t))-\widetilde{m}_{3}^{3} \partial_{3} \dot{\tilde{\zeta}}(t)-\widetilde{p}_{3}^{3} \partial_{3} \dot{\tilde{\varphi}}(t)+\widetilde{c}_{v}^{3} \tilde{\tilde{\theta}}(t)\right) \eta+ \\
&\left.+\widetilde{K}_{\alpha \beta}^{1} \partial_{\beta} \tilde{\theta}(t) \partial_{\alpha} \eta+\rho \tilde{\tilde{u}}_{i}(t) v_{i}\right\} d x \\
& \widetilde{L}_{3}(\mathcal{V}):=(\mathbf{f}, \mathbf{v})+(\mathbf{g}, \mathbf{v})_{\mathbf{L}^{2}(\widehat{\Gamma})}+(r, \eta)-(\varrho, \eta)_{L^{2}(\widehat{\Gamma})}+\left(\rho_{e}, \psi\right) .
\end{aligned}
$$

The reduced magneto-electro-thermo-elastic coefficients $\widetilde{C}_{\alpha \beta \sigma \tau}^{3}, \widetilde{X}_{33}^{3}, \widetilde{M}_{33}^{3}, \widetilde{P}_{3 \alpha \beta}^{3}, \widetilde{\beta}_{\alpha \beta}^{3}, \widetilde{p}_{3}^{3}$, $\widetilde{m}_{3}^{3}, \widetilde{R}_{3 \alpha \beta}^{3}, \widetilde{\alpha}_{33}^{3}$ and $\widetilde{c}_{v}^{3}$ are listed in Appendix 1.

\subsubsection{The limit evolution problem}

The primary unknowns $\tilde{\mathcal{U}}=(\tilde{\mathbf{u}}, \tilde{\varphi}, \tilde{\zeta}, \tilde{\theta}) \in \mathbf{V}_{K L}(\Omega) \times \mathbb{X}(\Omega) \times \mathbb{X}(\Omega) \times H^{1}\left(\Omega, \Gamma_{0}\right)$ are defined by:

$$
\begin{aligned}
& \tilde{u}_{\alpha}\left(\tilde{x}, x_{3}\right)=u_{\alpha}(\tilde{x})-x_{3} \partial_{\alpha} u_{3}(\tilde{x}), \quad \mathbf{u}_{H}:=\left(u_{\alpha}\right), \\
& \tilde{u}_{3}\left(\tilde{x}, x_{3}\right)=u_{3}(\tilde{x}), \\
& \tilde{\theta}\left(\tilde{x}, x_{3}\right)=\vartheta(\tilde{x}) .
\end{aligned}
$$

We consider the case of a homogeneous material. The limit evolution problem decouples into two variational subproblems, namely, the flexural problem, which gives $u_{3}$, and the thermo-elastic membrane problem, which gives the couple $\left(\mathbf{u}_{H}, \vartheta\right)$. Moreover, the characterization of the limit electric and magnetic potentials $\tilde{\varphi}$ and $\tilde{\zeta}$ is:

$$
\tilde{\varphi}\left(\tilde{x}, x_{3}\right)=\sum_{k=0}^{2} f^{k}(\tilde{x}) x_{3}^{k}, \quad \tilde{\zeta}\left(\tilde{x}, x_{3}\right)=\sum_{k=0}^{2} z^{k}(\tilde{x}) x_{3}^{k},
$$

where

$$
\begin{aligned}
& f^{0}=\langle\varphi\rangle+\frac{h^{2}}{2} \Lambda_{\alpha \beta} \partial_{\alpha \beta} u_{3}, \quad f^{1}=\frac{\llbracket \varphi \rrbracket}{2 h}, \quad f^{2}=-\frac{1}{2} \Lambda_{\alpha \beta} \partial_{\alpha \beta} u_{3}, \\
& z^{0}=\langle\zeta\rangle+\frac{h^{2}}{2} \Gamma_{\alpha \beta} \partial_{\alpha \beta} u_{3}, \quad z^{1}=\frac{\llbracket \zeta \rrbracket}{2 h}, \quad z^{2}=-\frac{1}{2} \Gamma_{\alpha \beta} \partial_{\alpha \beta} u_{3} .
\end{aligned}
$$

with

$$
\Lambda_{\alpha \beta}:=\frac{\widetilde{M}_{33}^{3} \widetilde{P}_{3 \alpha \beta}^{3}-\widetilde{\alpha}_{33}^{3} \widetilde{R}_{3 \alpha \beta}^{3}}{\widetilde{M}_{33}^{3} \widetilde{X}_{33}^{3}-\left(\widetilde{\alpha}_{33}^{3}\right)^{2}}, \quad \Gamma_{\alpha \beta}:=\frac{\widetilde{\alpha}_{33}^{3} \widetilde{P}_{3 \alpha \beta}^{3}-\widetilde{X}_{33}^{3} \widetilde{R}_{3 \alpha \beta}^{3}}{\widetilde{M}_{33}^{3} \widetilde{X}_{33}^{3}-\left(\widetilde{\alpha}_{33}^{3}\right)^{2}} .
$$

The limit magnetic and electric potentials depend on the transversal displacement $u_{3}$ of the plate and on the values of the known boundary magnetic potentials $\zeta^{ \pm}$and electric potentials $\varphi^{ \pm}$.

Thanks to the characterization (3.18) of the limit magnetic and electric potentials, we can now rewrite the limit evolution problem. After an integration by parts along $x_{3}$, we 
obtain a two-dimensional problem defined over the middle surface $\omega$ of the plate:

$$
\left\{\begin{array}{l}
\text { Find } \widetilde{\mathcal{U}}=\left(\mathbf{u}_{H}(t), u_{3}(t), \vartheta(t)\right) \in \mathbf{V}_{H}\left(\omega, \gamma_{0}\right) \times V_{3}\left(\omega, \gamma_{0}\right) \times H^{1}\left(\omega, \gamma_{0}\right), \text { such that } \\
2 h \int_{\omega}\left\{\mathcal{N}_{\alpha \beta}^{3}\left(\mathbf{u}_{H}(t), \vartheta(t)\right) e_{\alpha \beta}\left(\mathbf{v}_{H}\right)+\rho \ddot{u}_{\alpha}(t) v_{\alpha}\right\} d \tilde{x}+ \\
+2 h \int_{\omega}\left\{\dot{\mathcal{S}}^{3}\left(\mathbf{u}_{H}(t), \vartheta(t)\right) \eta-\widetilde{q}_{\alpha}^{1}(\vartheta(t)) \partial_{\alpha} \eta\right\} d \tilde{x}+ \\
\quad+\frac{2 h^{3}}{3} \int_{\omega}\left\{\mathcal{M}_{\alpha \beta}^{3}\left(u_{3}(t)\right) \partial_{\alpha \beta} v_{3}+\rho \partial_{\alpha} \ddot{u}_{3}(t) \partial_{\alpha} v_{3}+\frac{3}{h^{2}} \rho \ddot{u}_{3}(t) v_{3}\right\} d \tilde{x}=\mathcal{L}_{3}(\tilde{\mathcal{V}}), \\
\text { for all } \widetilde{\mathcal{V}}=\left(\mathbf{v}_{H}, v_{3}, \eta\right) \in \mathbf{V}_{H}\left(\omega, \gamma_{0}\right) \times V_{3}\left(\omega, \gamma_{0}\right) \times H^{1}\left(\omega, \gamma_{0}\right), t \in(0, T),
\end{array}\right.
$$

with

$$
\begin{aligned}
\mathcal{L}_{3}(\widetilde{\mathcal{V}}):= & \int_{\omega}\left\{\widetilde{f}_{i} v_{i}-m_{\alpha} \partial_{\alpha} v_{3}+\widetilde{\rho}_{e} \psi+\widetilde{r} \eta\right\} d \tilde{x}+\int_{\gamma_{1}}\left\{\widetilde{g}_{i} v_{i}-n_{\alpha} \partial_{\alpha} v_{3}-\widetilde{\varrho} \eta\right\} d \gamma- \\
& -\int_{\omega}\left\{\left(\widetilde{R}_{3 \alpha \beta}^{3} \llbracket \zeta \rrbracket+\widetilde{P}_{3 \alpha \beta}^{3} \llbracket \varphi \rrbracket\right) e_{\alpha \beta}\left(\mathbf{v}_{H}\right)-\left(\widetilde{p}_{3}^{3} \llbracket \dot{\varphi} \rrbracket+\widetilde{m}_{3}^{3} \llbracket \dot{\zeta} \rrbracket\right) \eta\right\} d \tilde{x} .
\end{aligned}
$$

The initial conditions are given by

$$
\left\{\begin{array}{l}
\tilde{\mathbf{u}}(0)=\tilde{\mathbf{u}}_{0}=\left(u_{\alpha, 0}-x_{3} \partial_{\alpha} u_{3,0}, u_{3,0}\right), \\
\dot{\tilde{\mathbf{u}}}(0)=\tilde{\mathbf{u}}_{1}=\left(u_{\alpha, 1}-x_{3} \partial_{\alpha} u_{3,1}, u_{3,1}\right), \\
\tilde{\theta}(0)=\tilde{\theta}_{0}=\vartheta_{0}
\end{array}\right.
$$

$\left(\mathcal{N}_{\alpha \beta}^{3}\right),\left(\mathcal{M}_{\alpha \beta}^{3}\right), \widetilde{\mathcal{S}}^{3}$ and $\left(\widetilde{q}_{\alpha}^{1}\right)$ represent, respectively, the membrane stress tensor, the moment tensor, the reduced thermodynamic entropy and the reduced heat flow vector of the plate, whose components are defined by the constitutive laws below:

$$
\left\{\begin{array}{l}
\mathcal{N}_{\alpha \beta}^{3}:=\widetilde{C}_{\alpha \beta \sigma \tau}^{3} e_{\sigma \tau}\left(\mathbf{u}_{H}\right)-\widetilde{\beta}_{\alpha \beta}^{3} \vartheta \\
\widetilde{\mathcal{S}}^{3}:=\widetilde{\beta}_{\alpha \beta}^{3} e_{\alpha \beta}\left(\mathbf{u}_{H}\right)+\widetilde{c}_{v}^{3} \vartheta \\
\mathcal{M}_{\alpha \beta}^{3}:=\widetilde{A}_{\alpha \beta \sigma \tau}^{3} \partial_{\sigma \tau} u_{3} \\
\widetilde{q}_{\alpha}^{1}:=-\frac{1}{T_{0}} \widetilde{K}_{\alpha \beta}^{1} \partial_{\beta} \vartheta
\end{array}\right.
$$

where $\widetilde{A}_{\alpha \beta \sigma \tau}^{3}:=\widetilde{C}_{\alpha \beta \sigma \tau}^{3}-\widetilde{P}_{3 \alpha \beta}^{3} \Lambda_{\sigma \tau}-\widetilde{R}_{3 \alpha \beta}^{3} \Gamma_{\sigma \tau}$.

The variational problem above can be split into the flexural problem and the thermoelastic membrane problem. The flexural problem reads as follows:

$$
\left\{\begin{array}{l}
\text { Find } u_{3}(t) \in V_{3}\left(\omega, \gamma_{0}\right), t \in(0, T) \text { such that } \\
\begin{array}{l}
\frac{2 h^{3}}{3} \int_{\omega}\left\{\mathcal{M}_{\alpha \beta}^{3}\left(u_{3}(t)\right) \partial_{\alpha \beta} v_{3}+\rho \partial_{\alpha} \ddot{u}_{3}(t) \partial_{\alpha} v_{3}+\frac{3}{h^{2}} \rho \ddot{u}_{3}(t) v_{3}\right\} d \tilde{x}= \\
\quad=\int_{\omega}\left\{\widetilde{f}_{3} v_{3}-m_{\alpha} \partial_{\alpha} v_{3}\right\} d \tilde{x}+\int_{\gamma_{1}}\left\{\widetilde{g}_{3} v_{3}-n_{\alpha} \partial_{\alpha} v_{3}\right\} d \gamma, \\
\text { for all } v_{3} \in V_{3}\left(\omega, \gamma_{0}\right) .
\end{array}
\end{array}\right.
$$


The two-dimensional thermo-elastic membrane problem takes the following form

$$
\left\{\begin{array}{l}
\text { Find }\left(\mathbf{u}_{H}(t), \vartheta(t)\right), \in \mathbf{V}_{H}\left(\omega, \gamma_{0}\right) \times H^{1}\left(\omega, \gamma_{0}\right), t \in(0, T) \text { such that } \\
2 h \int_{\omega}\left\{\mathcal{N}_{\alpha \beta}^{3}\left(\mathbf{u}_{H}(t), \vartheta(t)\right) e_{\alpha \beta}\left(\mathbf{v}_{H}\right)+\rho \ddot{u}_{\alpha}(t) v_{\alpha}\right\} d \tilde{x}+ \\
\quad+2 h \int_{\omega}\left\{\dot{\tilde{\mathcal{S}}}^{3}\left(\mathbf{u}_{H}(t), \vartheta(t)\right) \eta-\widetilde{q}_{\alpha}^{1}(\vartheta(t)) \partial_{\alpha} \eta\right\} d \tilde{x}= \\
\quad=\int_{\omega}\left\{\widetilde{f}_{\alpha} v_{\alpha}+\widetilde{\rho}_{e} \psi-\left(\widetilde{R}_{3 \alpha \beta}^{3} \llbracket \zeta \rrbracket+\widetilde{P}_{3 \alpha \beta}^{3} \llbracket \varphi \rrbracket\right) e_{\alpha \beta}\left(\mathbf{v}_{H}\right)+\left(\widetilde{r}+\widetilde{p}_{3}^{3} \llbracket \dot{\varphi} \rrbracket+\widetilde{m}_{3}^{3} \llbracket \dot{\zeta} \rrbracket\right) \eta\right\} d \tilde{x}+ \\
\quad+\int_{\gamma_{1}}\left\{\widetilde{g}_{\alpha} v_{\alpha}-\widetilde{\varrho} \eta\right\} d \gamma, \\
\text { for all }\left(\mathbf{v}_{H}, \eta\right) \in \mathbf{V}_{H}\left(\omega, \gamma_{0}\right) \times H^{1}\left(\omega, \gamma_{0}\right) .
\end{array}\right.
$$

By using Green's formulae on $\omega$, we can derive the differential formulations of the above problems. The transversal displacement $u_{3}$ solves a flexural differential problem $(F D P)^{3}$ analogous to $(F D P)^{1}$ with $\mathcal{M}_{\alpha \beta}^{1}$ replaced by $\mathcal{M}_{\alpha \beta}^{3}$ defined in $(3.21)_{3}$.

The thermo-elastic state $\left(\mathbf{u}_{H}(t), \vartheta(t)\right)$ solves the following thermo-elastic membrane differential problem:

$$
\begin{cases}\text { Field equations: } & \\ 2 h\left(\rho \ddot{u}_{\alpha}-\partial_{\beta} \mathcal{N}_{\alpha \beta}^{3}\right)=\widetilde{f}_{\alpha}+\widetilde{R}_{3 \alpha \beta}^{3} \llbracket \partial_{\beta} \zeta \rrbracket+\widetilde{P}_{3 \alpha \beta}^{3} \llbracket \partial_{\beta} \varphi \rrbracket & \text { in } \omega \times(0, T), \\ 2 h\left(\dot{\overrightarrow{\mathcal{S}}}^{3}+\partial_{\alpha} \widetilde{q}_{\alpha}^{1}\right)=\widetilde{r}+\widetilde{m}_{3}^{3} \llbracket \dot{\zeta} \rrbracket+\widetilde{p}_{3}^{3} \llbracket \dot{\varphi} \rrbracket & \text { in } \omega \times(0, T), \\ \text { Initial conditions: } & \\ u_{\alpha}(0)=u_{\alpha, 0}, \dot{u}_{\alpha}(0)=u_{\alpha, 1}, \vartheta(0)=\vartheta_{0} & \text { in } \omega \times(0, T), \\ \text { Boundary conditions: } & \\ 2 h \mathcal{N}_{\alpha \beta}^{3} \nu_{\beta}=\widetilde{g}_{\alpha}-\widetilde{R}_{3 \alpha \beta}^{3} \nu_{\beta} \llbracket \zeta \rrbracket-\widetilde{P}_{3 \alpha \beta}^{3} \nu_{\beta} \llbracket \varphi \rrbracket & \text { on } \gamma_{1} \times(0, T), \\ 2 h \widetilde{q}_{\alpha}^{1} \nu_{\alpha}=\widetilde{\varrho} & \text { on } \gamma_{1} \times(0, T), \\ u_{\alpha}=\vartheta=0 & \text { on } \gamma_{0} \times(0, T) .\end{cases}
$$

As in the sensor-actuator problem, the piezomagnetic and piezoelectric behaviors are contained in the load terms on the right-hand side of the equations.

\subsection{The Sensor Model}

Theorem 3.5. Under assumption (3.2), the sequence $\{\mathcal{U}(\varepsilon)\}_{\varepsilon>0}$ weakly converges to the limit $\tilde{\mathcal{U}}:=(\tilde{\mathbf{u}}, \tilde{\varphi}, \tilde{\zeta}, \tilde{\theta})$ in the space $L^{2}\left(0, T ; \mathbf{H}^{1}(\Omega)\right) \times L^{2}\left(0, T ; H^{1}(\Omega)\right) \times L^{2}\left(0, T ; H^{1}(\Omega)\right) \times$ $L^{2}\left(0, T ; H^{1}(\Omega)\right)$.

Theorem 3.6. The weak limit $\tilde{\mathcal{U}}(t)=(\tilde{\mathbf{u}}(t), \tilde{\varphi}(t), \tilde{\zeta}(t), \tilde{\theta}(t))$ is the solution to the limit variational problem:

$$
\left\{\begin{array}{l}
\text { Find } \widetilde{\mathcal{U}}(t) \in \mathbf{V}_{K L}(\Omega) \times H^{1}\left(\Omega, \Gamma_{0}\right) \times H^{1}\left(\Omega, \Gamma_{0}\right) \times H^{1}\left(\Omega, \Gamma_{0}\right), t \in(0, T) \text { such that } \\
\widetilde{A}_{4}(\widetilde{\mathcal{U}}(t), \mathcal{V})=\widetilde{L}_{4}(\mathcal{V}), \\
\text { for all } \mathcal{V} \in \mathbf{V}_{K L}(\Omega) \times H^{1}\left(\omega, \gamma_{0}\right) \times H^{1}\left(\omega, \gamma_{0}\right) \times H^{1}\left(\omega, \gamma_{0}\right),
\end{array}\right.
$$


where

$$
\begin{aligned}
& \widetilde{A}_{4}(\tilde{\mathcal{U}}(t), \mathcal{V}):=\int_{\Omega}\{\left(\widetilde{C}_{\alpha \beta \sigma \tau}^{4} e_{\sigma \tau}(\tilde{\mathbf{u}}(t))+\widetilde{P}_{\sigma \alpha \beta}^{4} \partial_{\sigma} \tilde{\varphi}(t)+\widetilde{R}_{\sigma \alpha \beta}^{4} \partial_{\sigma} \partial_{\sigma} \tilde{\zeta}(t)-\widetilde{\beta}_{\alpha \beta}^{4} \tilde{\theta}(t)\right) e_{\alpha \beta}(\mathbf{v})+ \\
&+\left(-\widetilde{P}_{\alpha \sigma \tau}^{4} e_{\sigma \tau}(\tilde{\mathbf{u}}(t))+\widetilde{X}_{\alpha \beta}^{4} \partial_{\beta} \tilde{\varphi}(t)+\widetilde{\alpha}_{\alpha \beta}^{4} \partial_{\beta} \tilde{\zeta}(t)-\widetilde{p}_{\alpha}^{4} \tilde{\theta}(t)\right) \partial_{\alpha} \psi+ \\
&+\left(-\widetilde{R}_{\alpha \sigma \tau}^{4} e_{\sigma \tau}(\tilde{\mathbf{u}}(t))+\widetilde{\alpha}_{\alpha \beta}^{4} \partial_{\beta} \tilde{\varphi}(t)+\widetilde{M}_{\alpha \beta}^{4} \partial_{\beta} \tilde{\zeta}(t)-\widetilde{m}_{\alpha}^{4} \tilde{\theta}(t)\right) \partial_{\alpha} \xi+ \\
&+\left(\widetilde{\beta}_{\alpha \beta}^{4} e_{\alpha \beta}(\dot{\tilde{\mathbf{u}}}(t))-\widetilde{m}_{\alpha}^{4} \partial_{\alpha} \dot{\tilde{\zeta}}(t)-\widetilde{p}_{\alpha}^{4} \partial_{\alpha} \dot{\tilde{\varphi}}(t)+\widetilde{c}_{v}^{4} \dot{\tilde{\theta}}(t)\right) \eta+ \\
&\left.+\widetilde{K}_{\alpha \beta}^{1} \partial_{\beta} \tilde{\theta}(t) \partial_{\alpha} \eta+\rho \ddot{\tilde{u}}_{i}(t) v_{i}\right\} d x \\
& \widetilde{L}_{4}(\mathcal{V}):=(\mathbf{f}, \mathbf{v})+(\mathbf{g}, \mathbf{v})_{\mathbf{L}^{2}(\widehat{\Gamma})}+(r, \eta)-(\varrho, \eta)_{L^{2}(\widehat{\Gamma})}+\left(\rho_{e}, \psi\right)-(d, \psi)_{L^{2}(\widehat{\Gamma})}-(b, \xi)_{L^{2}(\widehat{\Gamma})} .
\end{aligned}
$$

The reduced magneto-electro-thermo-elastic coefficients $\widetilde{C}_{\alpha \beta \sigma \tau}^{4}, \widetilde{X}_{\alpha \beta}^{4}, \widetilde{K}_{\alpha \beta}^{1}, \widetilde{P}_{\sigma \alpha \beta}^{4}, \widetilde{\beta}_{\alpha \beta}^{4}$, $\widetilde{p}_{\alpha}^{4} \widetilde{m}_{\alpha}^{4}, \widetilde{R}_{\sigma \alpha \beta}^{4}, \widetilde{M}_{\alpha \beta}^{4}, \widetilde{\alpha}_{\alpha \beta}^{4}$ and $\widetilde{c}_{v}^{4}$ are listed in Appendix 1.

\subsubsection{The limit evolution problem}

In this section we present the variational and differential formulations of the evolution problem for a sensor magneto-electro-thermo-elastic plate. The primary unknowns $\widetilde{\mathcal{U}}(t)=$ $(\tilde{\mathbf{u}}(t), \tilde{\varphi}(t), \tilde{\zeta}(t), \tilde{\theta}(t)) \in \mathbf{V}_{K L}(\Omega) \times H^{1}\left(\Omega, \Gamma_{0}\right) \times H^{1}\left(\Omega, \Gamma_{0}\right) \times H^{1}\left(\Omega, \Gamma_{0}\right)$ satisfy the following kinematical assumptions

$$
\begin{aligned}
& \tilde{u}_{\alpha}\left(\tilde{x}, x_{3}\right)=u_{\alpha}(\tilde{x})-x_{3} \partial_{\alpha} u_{3}(\tilde{x}), \quad \mathbf{u}_{H}:=\left(u_{\alpha}\right), \\
& \tilde{u}_{3}\left(\tilde{x}, x_{3}\right)=u_{3}(\tilde{x}), \\
& \tilde{\varphi}\left(\tilde{x}, x_{3}\right)=\phi(\tilde{x}) \\
& \tilde{\zeta}\left(\tilde{x}, x_{3}\right)=\varsigma(\tilde{x}) \\
& \tilde{\theta}\left(\tilde{x}, x_{3}\right)=\vartheta(\tilde{x}) .
\end{aligned}
$$

The limit evolution problem for a homogeneous material decouples into the flexural problem, which gives $u_{3}$, and the magneto-electro-thermo-elastic membrane problem, which gives the quadruplet $\left(\mathbf{u}_{H}, \phi, \varsigma, \vartheta\right)$. After an integration by parts along $x_{3}$, we obtain a two-dimensional problem defined over the middle surface $\omega$ of the plate:

$$
\begin{aligned}
& \text { (Find }\left(\mathbf{u}_{H}(t), u_{3}(t), \phi(t), \varsigma(t), \vartheta(t)\right) \in \mathbf{V}_{H}\left(\omega, \gamma_{0}\right) \times V_{3}\left(\omega, \gamma_{0}\right) \times\left[H^{1}\left(\omega, \gamma_{0}\right)\right]^{3} \text {, such that } \\
& 2 h \int_{\omega}\left\{\mathcal{N}_{\alpha \beta}^{4}\left(\mathbf{u}_{H}(t), \phi(t), \varsigma(t), \vartheta(t)\right) e_{\alpha \beta}\left(\mathbf{v}_{H}\right)+\rho \ddot{u}_{\alpha}(t) v_{\alpha}\right\} d \tilde{x}+ \\
& -2 h \int_{\omega}\left\{\widetilde{D}_{\alpha}^{4}\left(\mathbf{u}_{H}(t), \phi(t), \varsigma(t), \vartheta(t)\right) \partial_{\alpha} \psi+\widetilde{B}_{\alpha}^{4}\left(\mathbf{u}_{H}(t), \phi(t), \varsigma(t), \vartheta(t)\right) \partial_{\alpha} \xi\right\} d \tilde{x}+ \\
& +2 h \int_{\omega}^{\omega}\left\{\dot{\mathcal{S}}^{4}\left(\mathbf{u}_{H}(t), \phi(t), \varsigma(t), \vartheta(t)\right) \eta-\widetilde{q}_{\alpha}^{1}(\vartheta(t)) \partial_{\alpha} \eta\right\} d \tilde{x}+ \\
& +\frac{2 h^{3}}{3} \int_{\omega}\left\{\mathcal{M}_{\alpha \beta}^{3}\left(u_{3}(t)\right) \partial_{\alpha \beta} v_{3}+\rho \partial_{\alpha} \ddot{u}_{3}(t) \partial_{\alpha} v_{3}+\frac{3}{h^{2}} \rho \ddot{u}_{3}(t) v_{3}\right\} d \tilde{x}=\mathcal{L}_{4}(\widetilde{\mathcal{V}}), \\
& \text { for all } \widetilde{\mathcal{V}}=\left(\mathbf{v}_{H}, v_{3}, \psi, \xi, \eta\right) \in \mathbf{V}_{H}\left(\omega, \gamma_{0}\right) \times V_{3}\left(\omega, \gamma_{0}\right) \times\left[H^{1}\left(\omega, \gamma_{0}\right)\right]^{3}, t \in(0, T) \text {, } \\
& \mathcal{L}_{4}(\widetilde{\mathcal{V}}):=\int_{\omega}\left\{\tilde{f}_{i} v_{i}-m_{\alpha} \partial_{\alpha} v_{3}+\tilde{\rho}_{e} \psi+\widetilde{r} \eta\right\} d \tilde{x}+\int_{\gamma_{1}}\left\{\tilde{g}_{i} v_{i}-n_{\alpha} \partial_{\alpha} v_{3}-\tilde{d} \psi-\widetilde{b} \xi-\widetilde{\varrho} \eta\right\} d \gamma .
\end{aligned}
$$

The initial conditions are given by

$$
\left\{\begin{array}{l}
\tilde{\mathbf{u}}(0)=\tilde{\mathbf{u}}_{0}=\left(u_{\alpha, 0}-x_{3} \partial_{\alpha} u_{3,0}, u_{3,0}\right) \\
\dot{\tilde{\mathbf{u}}}(0)=\tilde{\mathbf{u}}_{1}=\left(u_{\alpha, 1}-x_{3} \partial_{\alpha} u_{3,1}, u_{3,1}\right) \\
\tilde{\theta}(0)=\tilde{\theta}_{0}=\vartheta_{0}
\end{array}\right.
$$


$\left(\mathcal{N}_{\alpha \beta}^{4}\right),\left(\mathcal{M}_{\alpha \beta}^{4}\right),\left(\widetilde{D}_{\alpha}^{4}\right),\left(\widetilde{B}_{\alpha}^{4}\right), \widetilde{\mathcal{S}}^{4}$ and $\left(\widetilde{q}_{\alpha}^{1}\right)$ represent, respectively, the membrane stress tensor, the moment tensor, the reduced electric displacement vector, the reduced magnetic induction vector, the reduced thermodynamic entropy and the reduced heat flow vector of the plate, whose components are defined by the constitutive laws below:

$$
\begin{aligned}
& \mathcal{N}_{\alpha \beta}^{4}:=\widetilde{C}_{\alpha \beta \sigma \tau}^{4} e_{\sigma \tau}\left(\mathbf{u}_{H}\right)+\widetilde{P}_{\sigma \alpha \beta}^{4} \partial_{\sigma} \phi+\widetilde{R}_{\sigma \alpha \beta}^{4} \partial_{\sigma \varsigma}-\widetilde{\beta}_{\alpha \beta}^{4} \vartheta \\
& \widetilde{D}_{\alpha}^{4}:=\widetilde{P}_{\alpha \sigma \tau}^{4} e_{\sigma \tau}\left(\mathbf{u}_{H}\right)-\widetilde{X}_{\alpha \beta}^{4} \partial_{\beta} \phi-\alpha_{\alpha \beta}^{4} \partial_{\beta} \varsigma+\widetilde{p}_{\alpha}^{4} \vartheta \\
& \widetilde{B}_{\alpha}^{4}:=\widetilde{R}_{\alpha \sigma \tau}^{4} e_{\sigma \tau}\left(\mathbf{u}_{H}\right)-\widetilde{\alpha}_{\alpha \beta}^{4} \partial_{\beta} \phi-M_{\alpha \beta}^{4} \partial_{\beta} \varsigma+\widetilde{m}_{\alpha}^{4} \vartheta \\
& \widetilde{\mathcal{S}}^{4}:=\widetilde{\beta}_{\alpha \beta}^{4} e_{\alpha \beta}\left(\mathbf{u}_{H}\right)-\widetilde{p}_{\alpha}^{4} \partial_{\alpha} \phi-\widetilde{m}_{\alpha}^{4} \partial_{\alpha \varsigma}+\widetilde{c}_{v}^{4} \vartheta \\
& \mathcal{M}_{\alpha \beta}^{4}:=\widetilde{C}_{\alpha \beta \sigma \tau}^{4} \partial_{\sigma \tau} u_{3}, \\
& \widetilde{q}_{\alpha}^{1}:=-\frac{1}{T_{0}} \widetilde{K}_{\alpha \beta}^{1} \partial_{\beta} \vartheta
\end{aligned}
$$

As previously announced this variational problem can be split into the flexural problem and the magneto-electro-thermo-elastic membrane problem. The flexural problem reads:

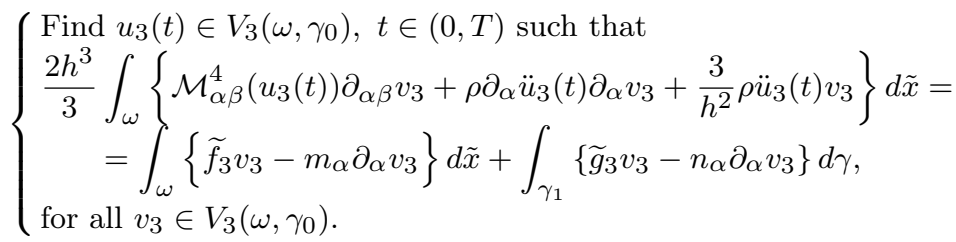

The two-dimensional magneto-electro-thermo-elastic membrane problem is:

$$
\left\{\begin{array}{l}
\text { Find }\left(\mathbf{u}_{H}(t), \phi(t), \varsigma(t), \vartheta(t)\right), \in \mathbf{V}_{H}\left(\omega, \gamma_{0}\right) \times\left[H^{1}\left(\omega, \gamma_{0}\right)\right]^{3}, t \in(0, T) \text { such that } \\
2 h \int_{\omega}\left\{\mathcal{N}_{\alpha \beta}^{4}\left(\mathbf{u}_{H}(t), \phi(t), \varsigma(t), \vartheta(t)\right) e_{\alpha \beta}\left(\mathbf{v}_{H}\right)+\rho \ddot{u}_{\alpha}(t) v_{\alpha}\right\} d \tilde{x}+ \\
\quad-2 h \int_{\omega}\left\{\widetilde{D}_{\alpha}^{4}\left(\mathbf{u}_{H}(t), \phi(t), \varsigma(t), \vartheta(t)\right) \partial_{\alpha} \psi+\widetilde{B}_{\alpha}^{4}\left(\mathbf{u}_{H}(t), \phi(t), \varsigma(t), \vartheta(t)\right) \partial_{\alpha} \xi\right\} d \tilde{x}+ \\
+2 h \int_{\omega}\left\{\dot{\widetilde{\mathcal{S}}}^{4}\left(\mathbf{u}_{H}(t), \phi(t), \varsigma(t), \vartheta(t)\right) \eta-\widetilde{q}_{\alpha}^{1}(\vartheta(t)) \partial_{\alpha} \eta\right\} d \tilde{x}= \\
=\int_{\omega}\left\{\widetilde{f}_{\alpha} v_{\alpha}+\widetilde{\rho}_{e} \psi+\widetilde{r} \eta\right\} d \tilde{x}+\int_{\gamma_{1}}\left\{\widetilde{g}_{\alpha} v_{\alpha}-\tilde{d} \psi-\tilde{b} \xi-\widetilde{\varrho} \eta\right\} d \gamma, \\
\text { for all }\left(\mathbf{v}_{H}, \psi, \xi, \eta\right) \in \mathbf{V}_{H}\left(\omega, \gamma_{0}\right) \times\left[H^{1}\left(\omega, \gamma_{0}\right)\right]^{3} .
\end{array}\right.
$$

By means of Green's formulae on $\omega$, we have that the transversal displacement $u_{3}$ solves a flexural differential problem $(F D P)^{4}$ analogous to $(F D P)^{1}$ with $\mathcal{M}_{\alpha \beta}^{1}$ replaced by $\mathcal{M}_{\alpha \beta}^{4}$ defined in $(3.28)_{5}$, whereas the membrane state $\left(\mathbf{u}_{H}(t), \phi(t), \varsigma(t), \vartheta(t)\right)$ solves the following 
magneto-electro-thermo-elastic membrane differential problem:

$$
\begin{cases}\text { Field equations: } & \\ 2 h\left(\rho \ddot{u}_{\alpha}-\partial_{\beta} \mathcal{N}_{\alpha \beta}^{4}\right)=\widetilde{f}_{\alpha} & \text { in } \omega \times(0, T), \\ 2 h \partial_{\alpha} \widetilde{D}_{\alpha}^{4}=\widetilde{\rho}_{e} & \text { in } \omega \times(0, T), \\ 2 h \partial_{\alpha} \widetilde{B}_{\alpha}^{4}=-\left(b^{+}+b^{-}\right) & \text {in } \omega \times(0, T), \\ 2 h\left(\dot{\tilde{\mathcal{S}}}^{4}+\partial_{\alpha} \widetilde{q}_{\alpha}^{1}\right)=\widetilde{r} & \text { in } \omega \times(0, T), \\ \text { Initial conditions: } & \\ u_{\alpha}(0)=u_{\alpha, 0}, \dot{u}_{\alpha}(0)=u_{\alpha, 1}, \vartheta(0)=\vartheta_{0} & \text { in } \omega \times(0, T), \\ \text { Boundary conditions: } & \text { on } \gamma_{1} \times(0, T), \\ 2 h \mathcal{N}_{\alpha \beta}^{4} \nu_{\beta}=\widetilde{g}_{\alpha} & \text { on } \gamma_{1} \times(0, T), \\ 2 h \widetilde{D}_{\alpha}^{4} \nu_{\alpha}=\widetilde{d} & \text { on } \gamma_{1} \times(0, T), \\ 2 h \widetilde{B}_{\alpha}^{4} \nu_{\alpha}=\widetilde{b} & \text { on } \gamma_{1} \times(0, T), \\ 2 h \widetilde{q}_{\alpha}^{1} \nu_{\alpha}=\widetilde{\varrho} & \text { on } \gamma_{0} \times(0, T) . \\ u_{\alpha}=\phi=\varsigma=\vartheta=0 & \end{cases}
$$

\section{Conclusions}

We set forth an asymptotic two-dimensional plate model for linear magneto-electrothermo-elastic sensors and actuators, under the hypotheses of homogeneity and anisotropy. A validation of the results obtained comes from the weak convergence theorems 3.1, 3.3 and 3.5. Each of the four plate problems originating from the four different boundary conditions presented decouples into a flexural problem and a partially or totally coupled membrane problem, depending on how the plate is supposed to behave. As in [14], the four models differ between one another in the scaling assumptions on the electric and magnetic potentials (see (2.4), (2.9), (2.12), (2.15)). The formulae in Appendix 1 giving the reduced material coefficients appear complex, however numerical values of such coefficients can be easily computed, as reported in Appendix 2 for the usual $\mathrm{BaTiO}_{3}-\mathrm{CoFe}_{2} \mathrm{O}_{4}$ composite.

Concerning directions for future research, a first important issue to deal with is, of course, coming up with efficient numerical methods to perform simulations, based either on the two-dimensional plate problems or on the three-dimensional problem. Then, another problem of interest is to extend our results to shell-like bodies; in situations where the geometry is particularly simple, as in the case of cylindrical shells, one should probably be able to determine analytical solutions by separation of variables, as in [12]. Finally, a further interesting problem is the study of a whole laminated structure (plate-like or shell-like) including a thin magneto-electro-thermo-elastic layer (see, e.g., [10]).

\section{Appendix 1}

In the sequel we define the reduced magneto-electro-thermo-elastic coefficients characterizing the different models. We recall that $\left(d_{i j}\right)$ is the inverse of a second-order tensor whose $i j$ components are $C_{i 3 j 3}$. The tensor $\left(\widetilde{K}_{\alpha \beta}^{1}\right)$ is the same throughout the models. In the list 
below, we give at first the definitions of the general reduced coefficients.

$$
\begin{array}{lll}
C_{\alpha \beta \sigma \tau}^{\prime}:=C_{\alpha \beta \sigma \tau}-d_{i j} C_{\alpha \beta i 3} C_{\sigma \tau j 3}, & R_{3 \alpha \beta}^{\prime}:=R_{3 \alpha \beta}-d_{i j} C_{\alpha \beta i 3} R_{3 j 3}, & K_{\alpha 3}^{\prime}:=\frac{K_{\alpha 3}}{K_{33}}, \\
X_{p 3}^{\prime}:=X_{p 3}+d_{i j} P_{3 i 3} P_{p j 3}, & \beta_{\alpha \beta}^{\prime}:=\beta_{\alpha \beta}-d_{i j} C_{\alpha \beta i 3} \beta_{j 3}, & K_{\alpha \beta}^{\prime}:=K_{\alpha \beta}-K_{\alpha 3}^{\prime} K_{\beta 3}, \\
M_{p 3}^{\prime}:=M_{p 3}+d_{i j} R_{3 i 3} R_{p j 3}, & p_{3}^{\prime}:=p_{3}+d_{i j} P_{3 j 3} \beta_{i 3}, & k^{\prime}:=\frac{1}{X_{33}^{\prime}}, \\
\alpha_{33}^{\prime}:=\alpha_{33}+d_{i j} P_{3 i 3} R_{3 j 3}, & m_{3}^{\prime}:=m_{3}+d_{i j} R_{3 i 3} \beta_{j 3}, & \ell^{\prime}:=\frac{1}{M_{33}^{\prime}}, \\
P_{p \alpha \beta}^{\prime}:=P_{p \alpha \beta}-d_{i j} P_{p i 3} C_{\alpha \beta j 3}, & c_{v}^{\prime}:=c_{v}+d_{i j} \beta_{i 3} \beta_{j 3}, & c^{\prime}:=\frac{1}{M_{33}^{\prime} X_{33}^{\prime}-\left(\alpha_{33}^{\prime}\right)^{2}},
\end{array}
$$

\section{Reduced Coefficients for the Sensor-Actuator Model}

$$
\begin{aligned}
& \widetilde{C}_{\alpha \beta \sigma \tau}^{1}:=C_{\alpha \beta \sigma \tau}-C_{\alpha \beta i 3} d_{i j}\left(C_{\sigma \tau j 3}+k^{\prime} P_{3 j 3} P_{3 \sigma \tau}^{\prime}\right)+k^{\prime} P_{3 \alpha \beta} P_{3 \sigma \tau}^{\prime}, \\
& \widetilde{P}_{\sigma \alpha \beta}^{1}:=P_{\sigma \alpha \beta}-C_{\alpha \beta i 3} d_{i j}\left(P_{\sigma j 3}-k^{\prime} P_{3 j 3} X_{\sigma 3}^{\prime}\right)-k^{\prime} P_{3 \alpha \beta} X_{\sigma 3}^{\prime}, \\
& \widetilde{R}_{3 \alpha \beta}^{1}:=R_{3 \alpha \beta}-C_{\alpha \beta i 3} d_{i j}\left(R_{3 j 3}-k^{\prime} P_{3 j 3} \alpha_{33}^{\prime}\right)-k^{\prime} P_{3 \alpha \beta} \alpha_{33}^{\prime}, \\
& \widetilde{\beta}_{\alpha \beta}^{1}:=\beta_{\alpha \beta}-C_{\alpha \beta i 3} d_{i j}\left(\beta_{j 3}-k^{\prime} P_{3 j 3} p_{3}^{\prime}\right)-k^{\prime} P_{3 \alpha \beta} p_{3}^{\prime}, \\
& \widetilde{X}_{\alpha \beta}^{1}:=X_{\alpha \beta}+P_{\alpha i 3} d_{i j}\left(P_{\beta j 3}-k^{\prime} P_{3 j 3} X_{\beta 3}^{\prime}\right)-k^{\prime} X_{\alpha 3} X_{\beta 3}^{\prime}, \\
& \widetilde{\alpha}_{\alpha 3}^{1}:=\alpha_{\alpha 3}+P_{\alpha i 3} d_{i j}\left(R_{3 j 3}-k^{\prime} P_{3 j 3} \alpha_{33}^{\prime}\right)-k^{\prime} X_{\alpha 3} \alpha_{33}^{\prime}, \\
& \widetilde{p}_{\alpha}^{1}:=p_{\alpha}+P_{\alpha i 3} d_{i j}\left(\beta_{j 3}-k^{\prime} P_{3 j 3} p_{3}^{\prime}\right)-k^{\prime} X_{\alpha 3} p_{3}^{\prime}, \\
& \widetilde{M}_{33}^{1}:=M_{33}+R_{3 i 3} d_{i j}\left(R_{3 j 3}-k^{\prime} P_{3 j 3} \alpha_{33}^{\prime}\right)-k^{\prime} \alpha_{33} \alpha_{33}^{\prime}, \\
& \widetilde{m}_{3}^{1}:=m_{3}+R_{3 i 3} d_{i j}\left(\beta_{j 3}-k^{\prime} P_{3 j 3} p_{3}^{\prime}\right)-k^{\prime} \alpha_{33} p^{\prime} 3, \\
& \widetilde{c}_{v}^{1}:=c_{v}+\beta_{i 3} d_{i j}\left(\beta_{j 3}-k^{\prime} P_{3 j 3} p_{3}^{\prime}\right)-k^{\prime} p_{3} p_{3}^{\prime} .
\end{aligned}
$$

\section{Reduced Coefficients for the Sensor Model}

$$
\begin{aligned}
& \widetilde{C}_{\alpha \beta \sigma \tau}^{4}:=C_{\alpha \beta \sigma \tau}-C_{\alpha \beta i 3}\left[d_{i j}^{\prime} C_{\sigma \tau j 3}+\left(\alpha_{33}^{\prime} R_{3 i 3}-M_{33}^{\prime} P_{3 i 3}\right) P_{3 \sigma \tau}+\left(\alpha_{33}^{\prime} P_{3 i 3}-X_{33}^{\prime} R_{3 i 3}\right) R_{3 \sigma \tau}\right]+ \\
& -c^{\prime} P_{3 \alpha \beta}\left[d_{i j}\left(M_{33}^{\prime} P_{3 j 3}-\alpha_{33}^{\prime} R_{3 j 3}\right) C_{\alpha \beta i 3}-M_{33}^{\prime} P_{3 \alpha \beta}+\alpha_{33}^{\prime} R_{3 \alpha \beta}\right]+ \\
& -c^{\prime} R_{3 \alpha \beta}\left[d_{i j}\left(X_{33}^{\prime} R_{3 j 3}-\alpha_{33}^{\prime} P_{3 j 3}\right) C_{\alpha \beta i 3}-X_{33}^{\prime} R_{3 \alpha \beta}+\alpha_{33}^{\prime} P_{3 \alpha \beta}\right] \text {, } \\
& \widetilde{P}_{\sigma \alpha \beta}^{4}:=P_{\sigma \alpha \beta}-C_{\alpha \beta i 3}\left[d_{i j}^{\prime} P_{\sigma j 3}-\left(\alpha_{33}^{\prime} R_{3 i 3}-M_{33}^{\prime} P_{3 i 3}\right) X_{\sigma 3}-\left(\alpha_{33}^{\prime} P_{3 i 3}-X_{33}^{\prime} R_{3 i 3}\right) \alpha_{\sigma 3}\right]+ \\
& -c^{\prime} P_{3 \alpha \beta}\left[d_{i j}\left(M_{33}^{\prime} P_{3 j 3}-\alpha_{33}^{\prime} R_{3 j 3}\right) P_{\sigma i 3}+M_{33}^{\prime} X_{\sigma 3}-\alpha_{33}^{\prime} \alpha_{\sigma 3}\right]+ \\
& -c^{\prime} R_{3 \alpha \beta}\left[d_{i j}\left(X_{33}^{\prime} R_{3 j 3}-\alpha_{33}^{\prime} P_{3 j 3}\right) P_{\sigma i 3}+X_{33}^{\prime} M_{\sigma 3}-\alpha_{33}^{\prime} X_{\sigma 3}\right] \\
& \widetilde{R}_{\sigma \alpha \beta}^{4}:=R_{\sigma \alpha \beta}-C_{\alpha \beta i 3}\left[d_{i j}^{\prime} R_{\sigma j 3}-\left(\alpha_{33}^{\prime} R_{3 i 3}-M_{33}^{\prime} P_{3 i 3}\right) \alpha_{\sigma 3}-\left(\alpha_{33}^{\prime} P_{3 i 3}-X_{33}^{\prime} R_{3 i 3}\right) M_{\sigma 3}\right]+ \\
& -c^{\prime} P_{3 \alpha \beta}\left[d_{i j}\left(M_{33}^{\prime} P_{3 j 3}-\alpha_{33}^{\prime} R_{3 j 3}\right) R_{\sigma i 3}+M_{33}^{\prime} \alpha_{\sigma 3}-\alpha_{33}^{\prime} M_{\sigma 3}\right]+ \\
& -c^{\prime} R_{3 \alpha \beta}\left[d_{i j}\left(X_{33}^{\prime} R_{3 j 3}-\alpha_{33}^{\prime} P_{3 j 3}\right) R_{\sigma i 3}+X_{33}^{\prime} M_{\sigma 3}-\alpha_{33}^{\prime} \alpha_{\sigma 3}\right] \text {, } \\
& \widetilde{\beta}_{\alpha \beta}^{4}:=\beta_{\alpha \beta}-C_{\alpha \beta i 3}\left[d_{i j}^{\prime} \beta_{j 3}+\left(\alpha_{33}^{\prime} R_{3 i 3}-M_{33}^{\prime} P_{3 i 3}\right) p_{3}+\left(\alpha_{33}^{\prime} P_{3 i 3}-X_{33}^{\prime} R_{3 i 3}\right) m_{3}\right]+ \\
& -c^{\prime} P_{3 \alpha \beta}\left[d_{i j}\left(M_{33}^{\prime} P_{3 j 3}+\alpha_{33}^{\prime} R_{3 j 3}\right) \beta_{i 3}-M_{33}^{\prime} p_{3}+\alpha_{33}^{\prime} m_{3}\right]+ \\
& -c^{\prime} R_{3 \alpha \beta}\left[d_{i j}\left(X_{33}^{\prime} R_{3 j 3}-\alpha_{33}^{\prime} P_{3 j 3}\right) \beta_{i 3}-X_{33}^{\prime} m_{3}+\alpha_{33}^{\prime} p_{3}\right] \text {, } \\
& \widetilde{X}_{\alpha \beta}^{4}:=X_{\alpha \beta}+P_{\alpha i 3}\left[d_{i j}^{\prime} P_{\beta j 3}-\left(\alpha_{33}^{\prime} R_{3 i 3}-M_{33}^{\prime} P_{3 i 3}\right) X_{\beta 3}-\left(\alpha_{33}^{\prime} P_{3 i 3}-X_{33}^{\prime} R_{3 i 3}\right) \alpha_{\beta 3}\right]+ \\
& -c^{\prime} X_{\alpha 3}\left[d_{i j}\left(M_{33}^{\prime} P_{3 j 3}-\alpha_{33}^{\prime} R_{3 j 3}\right) P_{\beta i 3}+M_{33}^{\prime} X_{\beta 3}-\alpha_{33}^{\prime} \alpha_{\beta 3}\right]+ \\
& -c^{\prime} \alpha_{\alpha 3}\left[d_{i j}\left(X_{33}^{\prime} R_{3 j 3}-\alpha_{33}^{\prime} P_{3 j 3}\right) P_{\beta i 3}+X_{33}^{\prime} M_{\beta 3}-\alpha_{33}^{\prime} X_{\beta 3}\right] \text {, } \\
& \widetilde{\alpha}_{\alpha \beta}^{4}:=\alpha_{\alpha \beta}+P_{\alpha i 3}\left[d_{i j}^{\prime} R_{\beta j 3}-\left(\alpha_{33}^{\prime} R_{3 i 3}-M_{33}^{\prime} P_{3 i 3}\right) \alpha_{\beta 3}-\left(\alpha_{33}^{\prime} P_{3 i 3}-X_{33}^{\prime} R_{3 i 3}\right) M_{\beta 3}\right]+ \\
& -c^{\prime} X_{\alpha 3}\left[d_{i j}^{\prime} R_{\beta j 3}-\left(\alpha_{33}^{\prime} R_{3 i 3}-M_{33}^{\prime} P_{3 i 3}\right) \alpha_{\beta 3}-\left(\alpha_{33}^{\prime} P_{3 i 3}-X_{33}^{\prime} R_{3 i 3}\right) M_{\beta 3}\right]+ \\
& -c^{\prime} \alpha_{\alpha 3}\left[d_{i j}\left(X_{33}^{\prime} R_{3 j 3}-\alpha_{33}^{\prime} P_{3 j 3}\right) R_{\alpha i 3}+X_{33}^{\prime} M_{\alpha 3}-\alpha_{33}^{\prime} \alpha_{\alpha 3}\right] \text {, }
\end{aligned}
$$




$$
\begin{gathered}
\widetilde{p}_{\alpha}^{4}:=p_{\alpha}-P_{\alpha i 3}\left[d_{i j}^{\prime} \beta_{j 3}+\left(\alpha_{33}^{\prime} R_{3 i 3}-M_{33}^{\prime} P_{3 i 3}\right) p_{3}+\left(\alpha_{33}^{\prime} P_{3 i 3}-X_{33}^{\prime} R_{3 i 3}\right) m_{3}\right]+ \\
-c^{\prime} X_{\alpha 3}\left[d_{i j}\left(M_{33}^{\prime} P_{3 j 3}-\alpha_{33}^{\prime} R_{3 j 3}\right) \beta_{i 3}-M_{33}^{\prime} p_{3}+\alpha_{33}^{\prime} m_{3}\right]+ \\
-c^{\prime} \alpha_{\alpha 3}\left[d_{i j}\left(X_{33}^{\prime} R_{3 j 3}+\alpha_{33}^{\prime} P_{3 j 3}\right) \beta_{i 3}-X_{33}^{\prime} m_{3}+\alpha_{33}^{\prime} p_{3}\right] \\
\widetilde{M}_{\alpha \beta}^{4}:=M_{\alpha \beta}+R_{\alpha i 3}\left[d_{i j}^{\prime} R_{\beta j 3}-\left(\alpha_{33}^{\prime} R_{3 i 3}-M_{33}^{\prime} P_{3 i 3}\right) \alpha_{33}-\left(\alpha_{33}^{\prime} P_{3 i 3}-X_{33}^{\prime} R_{3 i 3}\right) M_{\beta 3}\right]+ \\
-c^{\prime} \alpha_{\alpha 3}\left[d_{i j}\left(M_{33}^{\prime} P_{3 j 3}-\alpha_{33}^{\prime} R_{3 j 3}\right) R_{\beta i 3}+M_{33}^{\prime} \alpha_{\beta 3}-\alpha_{33}^{\prime} M_{\beta 3}\right]+ \\
-c^{\prime} M_{\alpha 3}\left[d_{i j}\left(X_{33}^{\prime} R_{3 j 3}-\alpha_{33}^{\prime} P_{3 j 3}\right) R_{\beta i 3}+X_{33}^{\prime} M_{\beta 3}-\alpha_{33}^{\prime} \alpha_{\beta 3}\right], \\
\widetilde{m}_{\alpha}^{4}:=m_{\alpha}-R_{\alpha i 3}\left[d_{i j}^{\prime} \beta_{j 3}-\left(\alpha_{33}^{\prime} R_{3 i 3}+M_{33}^{\prime} P_{3 i 3}\right) p_{3}+\left(\alpha_{33}^{\prime} P_{3 i 3}-X_{33}^{\prime} R_{3 i 3}\right) m_{3}\right]+ \\
-c^{\prime} \alpha_{\alpha 3}\left[d_{i j}\left(M_{33}^{\prime} P_{3 j 3}-\alpha_{33}^{\prime} R_{3 j 3}\right) \beta_{i 3}-M_{33}^{\prime} p_{3}+\alpha_{33}^{\prime} m_{3}\right]+ \\
-c^{\prime} M_{\alpha 3}\left[d_{i j}\left(X_{33}^{\prime} R_{3 j 3}-\alpha_{33}^{\prime} P_{3 j 3}\right) \beta_{i 3}-X_{33}^{\prime} m_{3}+\alpha_{33}^{\prime} p_{3}\right] \\
\left.\widetilde{c}_{v}^{4}:=c_{v}+\beta_{i 3}\left[d_{i j}^{\prime} \beta_{j 3}+\left(\alpha_{33}^{\prime} R_{3 i 3}-M_{33}^{\prime} P_{3 i 3}\right) p_{3}+\alpha_{33}^{\prime} P_{3 i 3}-X_{33}^{\prime} R_{3 i 3}\right) m_{3}\right]+ \\
-c^{\prime} p_{3}\left[d_{i j}\left(M_{33}^{\prime} P_{3 j 3}-\alpha_{33}^{\prime} R_{3 j 3}\right) \beta_{i 3}-M_{33}^{\prime} p_{3}+\alpha_{33}^{\prime} m_{3}\right]+ \\
-c^{\prime} m_{3}\left[d_{i j}\left(X_{33}^{\prime} R_{3 j 3}-\alpha_{33}^{\prime} P_{3 j 3}\right) \beta_{i 3}-X_{33}^{\prime} m_{3}+\alpha_{33}^{\prime} p_{3}\right] .
\end{gathered}
$$

\begin{tabular}{|c|c|c|c|}
\hline $\begin{array}{c}\text { Elastic moduli } \\
\widetilde{C}_{1111}^{3}=\widetilde{C}_{2222}^{2}(\mathrm{GPa})\end{array}$ & 136 & $\begin{array}{l}\text { Magnetic permeability } \\
\widetilde{M}_{33}^{3}\left(10^{-4} \mathrm{~N} \mathrm{~s}^{2} / \mathrm{C}^{2}\right)\end{array}$ & 0.75 \\
\hline $\begin{array}{l}\widetilde{C}_{1122}^{3}(\mathrm{GPa}) \\
\widetilde{C}_{1212}^{3}(\mathrm{GPa})\end{array}$ & 45 & $\begin{array}{c}\text { Piezomagnetic constants } \\
\widetilde{R}_{311}^{3}=\widetilde{R}_{322}^{3}(\mathrm{~N} / \mathrm{A} \mathrm{m})\end{array}$ & 49.5 \\
\hline $\begin{array}{c}\text { Piezoelectric constants } \\
\widetilde{P}_{311}^{3}=\widetilde{P}_{322}^{3}\left(\mathrm{C} / \mathrm{m}^{2}\right)\end{array}$ & -9.86 & $\begin{array}{l}\text { Magnetoelectric constant } \\
\qquad \widetilde{\alpha}_{33}^{3}\left(10^{-8} \mathrm{~N} \mathrm{~s} / \mathrm{V} \mathrm{C}\right)\end{array}$ & 1.75 \\
\hline $\begin{array}{l}\text { Dielectric permittivity } \\
\qquad \widetilde{X}_{33}^{3}\left(10^{-9} \mathrm{C}^{2} / \mathrm{N} \mathrm{m}^{2}\right)\end{array}$ & 8.1 & $\begin{array}{l}\text { Pyroelectric constant } \\
\widetilde{p}_{3}^{3}\left(10^{-4} \mathrm{C} / \mathrm{m}^{2} \mathrm{~K}\right)\end{array}$ & 2.5 \\
\hline \multirow[t]{2}{*}{$\begin{array}{c}\text { Thermal stresses } \\
\widetilde{\beta}_{11}^{3}=\widetilde{\beta}_{22}^{3}\left(10^{6} \mathrm{~N} / \mathrm{K} \mathrm{m}^{2}\right)\end{array}$} & \multirow[t]{2}{*}{2.36} & $\begin{array}{l}\text { Pyromagnetic constant } \\
\widetilde{m}_{3}^{3}\left(10^{-3} \mathrm{~N} / \mathrm{A} \mathrm{m} \mathrm{K}\right)\end{array}$ & 5.91 \\
\hline & & $\begin{array}{l}\text { Calorific capacity } \\
\qquad \widetilde{c}_{v}^{3}\left(\mathrm{~J} / \mathrm{m}^{3} \mathrm{~K}^{2}\right)\end{array}$ & 423 \\
\hline
\end{tabular}

\section{Appendix 2}

\section{Numerical Values of Reduced Material Coefficients}

The table below shows the calculated numerical values (based on Table 1 in [2]) of the reduced coefficients in the case of a plate behaving as an actuator, both piezoelectric and piezomagnetic. One can analogously obtain such values also in the other three cases. In this particular case, since $K_{33}$ is the only nonzero component of $\mathbf{K}$, the reduced thermal conductivity tensor $\left(\widetilde{K}_{\alpha \beta}\right)$ is null; this holds true for the other three models as well, since the expression of $\widetilde{K}_{\alpha \beta}$ is unmodified, as pointed out previously.

Table 1. Reduced coefficients for a magneto-electro-thermo-elastic actuator made up of a $\mathrm{BaTiO}_{3}-$ $\mathrm{CoFe}_{2} \mathrm{O}_{4}$ composite with 0.6 volume fraction of $\mathrm{BaTiO}_{3}$. 


\section{Acknowledgements}

This work has been supported by the French Agence Nationale de la Recherche (ANR) under grant ARAMIS (Projet «Blanc», N. ANR 12 BS01-0021) (Analysis of Robust Asymptotic Methods In numerical Simulation in mechanics).

\section{References}

[1] D. Blanchard, G. A. Francfort, Asymptotic thermoelastic behavior of flat plates, Quart. Appl. Math., Vol. XLV, No. 4 (1987), 645-667.

[2] F. Bonaldi, G. Geymonat, F. Krasucki, Modeling of smart materials with thermal effects: dynamic and quasi-static evolution, to appear in Math. Models Methods Appl. Sci. (2015).

[3] P. G. Ciarlet, Mathematical Elasticity, vol. II: Theory of Plates, North-Holland, Amsterdam (1997).

[4] P. Grisvard, Elliptic Problems in Nonsmooth Domains, SIAM (2011).

[5] J. Y. Kim, Z. Li, A. Baltazar, Pyroelectric and pyromagnetic coefficients of functionally graded multilayered multiferroic composites, Acta Mech. 223 (2012), 849-860.

[6] J. L. Lions, Equations différentielles opérationnelles et problèmes aux limites, Springer Verlag, Berlin (1961).

[7] B. Miara, J. S. Suárez, Asymptotic pyroelectricity and pyroelasticity in thermopiezoelectric plates, Asymptot. Anal. 81 (2013), 211-250.

[8] P. Podio-Guidugli, Inertia and Invariance, Ann Mat Pur Appl (IV), Vol. CLXXII (1997), 103-124.

[9] P. Podio-Guidugli, S. Sellers, G. Vergara Caffarelli, On the representation of energy and momentum in elasticity, Math. Models Methods Appl. Sci. 10(2) (1999).

[10] M. Serpilli, Mathematical Modeling of Weak and Strong Piezoelectric Interfaces, J Elasticity (2015), DOI:10.1007/s10659-015-9526-5, published online.

[11] A. Sène, Modelling of piezoelectric static thin plates, Asymptot. Anal. 25 (2001), $1-20$.

[12] X. Wang, Z. Zhong, A finitely long circular cylindrical shell of piezoelectric/piezomagnetic composite under pressuring and temperature change, Internat. J. Engrg. Sc. 41 (2003), 2429-2445.

[13] T. Weller, C. Licht, Réponse dynamique asymptotique de plaques minces linéairement piézoélectriques dans l'approximation quasi-électrostatique, C. R. Mécanique Acad. Sci Paris 332 (2004), 519-524.

[14] T. Weller, C. Licht, Modeling of linearly electromagneto-elastic thin plates, C. R. Mécanique Acad. Sci Paris 335, (2007), 201-206.

[15] T. Weller, C. Licht, Asymptotic modeling of thin piezoelectric plates, Ann Solid Struct Mech 1, (2010), 173-188.

[16] T. Weller, C. Licht, Mathematical modeling of piezomagnetoelectric thin plates. Eur. J. Mech. A Solids 29, (2010) 928-937.

[17] T. Weller, C. Licht, Asymptotic modeling of piezoelectric plates with electric field gradient, C. R. Mécanique Acad. Sci Paris 340 (2012) 405-410. 